\title{
REPORT ON THE PROGRESS OF THE SOLUTION OF CERTAIN SPECIAL PROBLEMS OF DYNAMICS.
}

[From the Report of the British Association for the Advancement of Science, 1862, pp. $184-252$.

My "Report on the Recent Progress of Theoretical Dynamics" was published in the Report of the British Association for the year 1857, [195]. The present Report (which is in some measure supplemental thereto) relates to the Special Problems of Dynamics: - to give a general idea of the contents, I will at once mention the heads under which these problems are considered; viz., relating to the motion of a particle or system of particles, we have

Rectilinear Motion;

Central Forces, and in particular

Elliptic Motion;

The Problem of two Centres;

The Spherical Pendulum;

Motion as affected by the Rotation of the Earth, and Relative Motion in general;

Miscellaneous Problems;

The Problem of three bodies.

And relating to the motion of a solid body, we have

The Transformation of Coordinates;

Principal Axes, and Moments of Inertia;

Rotation of a Solid Body;

Kinematics of a Solid Body;

Miscellaneous Problems.

C. IV. 
As regards the first division of the subject, I remark that the lunar and planetary theories, as usually treated, do not (properly speaking) relate to the problem of three bodies, but to that of disturbed elliptic motion-a problem which is not considered in the present Report. The problem of the spherical pendulum is that of a particle moving on a spherical surface; but, with this exception, I do not much consider the motion of a particle on a given curve or surface, nor the motion in a resisting medium; what is said on these subjects is included under the head Miscellaneous Problems. The first six heads relate exclusively, and the head Miscellaneous Problems relates principally, to the motion of a single particle. As regards the second division of the subject, I will only remark that, from its intimate connexion with the theory of the motion of a solid body, I have been induced to make a separate head of the geometrical subject, "Transformation of Coordinates," and to treat of it in considerable detail.

I have inserted at the end of the present Report a list of the memoirs and works referred to, arranged (not, as in the former Report, in chronological order, but) alphabetically according to the authors' names: those referred to in the former Report formed for the purpose thereof a single series, which is not here the case. The dates specified are for the most part those on the title-page of the volume, being intended to show approximately the date of the researches to which they refer, but in some instances a more particular specification is made.

I take the opportunity of noticing a serious omission in my former Report, viz., I have not made mention of the elaborate memoir, Ostrogradsky "Mémoire sur les équations différentielles relatives au problème des Isopérimètres," Mém. de St Pét., t. IV. (6 sér.) pp. 385-517, 1850, which among other researches contains, and that in the most general form, the transformation of the equations of motion from the Lagrangian to the Hamiltonian form, and indeed the transformation of the general isoperimetric system (that is, the system arising from any problem in the calculus of variations) to the Hamiltonian form. I remark also, as regards the memoir of Cauchy referred to in the note p. 12 as an unpublished memoir of 1831, there is an "Extrait du Mémoire présenté à l'Académie de Turin le 11 Oct. 1831," published in lithograph under the date Turin, 1832, with an addition dated 6 Mar. 1833. The Extract begins thus :"§ I. Variation des Constantes Arbitraires. Soient données entre la variable $t, \ldots$ $n$ fonctions de $t$ désignées par $x, y, z$.. et $n$ autres fonctions de $t$ désignées par $u, v, w, \ldots$ $2 n$ équations différentielles du premier ordre et de la forme

$$
\begin{aligned}
& \frac{d x}{d t}=\frac{d Q}{d u}, \quad \frac{d y}{d t}=\frac{d Q}{d v}, \quad \frac{d z}{d t}=\frac{d Q}{d w}, \\
& \frac{d u}{d t}=-\frac{d Q}{d x}, \quad \frac{d v}{d t}=-\frac{d Q}{d y}, \quad \frac{d w}{d t}=-\frac{d Q}{d z}, \& c . "
\end{aligned}
$$

without any explanation as to the origin of these equations; and the formulæ are then given for the variations of the constants in the integrals of the foregoing system; this seems sufficient to establish that Cauchy in the year 1831 was familiar with the Hamiltonian form of the equations of motion. 
Bour's "Mémoire sur l'intégration des équations différentielles de la Mécanique," as published, Mém. prés. à l'Inst., t. xIv. pp. 792-821, is substantially the same as the extract thereof in Liouville's Journal, referred to in my former Report; but since the date of that Report there have been published in the Comptes Rendus, 1861 and 1862, several short papers by the same author; also Jacobi's great memoir, see list, Jacobi, Nova Methodus \&c., 1862, edited after his decease by Clebsch; some valuable memoirs by Natani and Clebsch (Crelle, 1861 and 1862) relating to the Pfaffian system of equations (which includes those of Dynamics), and Boole "On Simultaneous Differential Equations of the First Order, in which the number of the Variables exceeds by more than one the number of the Equations," Phil. Trans., t. CLII. (1862), pp. 437-454.

\section{Rectilinear Motion. Article Nos. 1 to 5.}

1. The determination of the motion of a falling body, which is the case of a constant force, is due to Galileo.

2. A variable force, assumed to be a force depending only on the position of the particle, may be considered as a function of the distance from any point in the line, selected at pleasure as a centre of force; but if, as usual, the force is given as a function of the distance from a certain point, it is natural to take that point for the centre of force. The problem thus becomes a particular case of that of central forees; and it is so treated in the Principia, Book I. $\S 7$; the method has the advantage of explaining the paradoxical result which presents itself in the case Force $\propto$ (Dist. $)^{-2}$, and in some other cases where the force becomes infinite. According to theory, the velocity becomes infinite at the centre, but the direction of the motion is there abruptly reversed; so that the body in its motion does not pass through the centre, but on arriving there, forthwith returns towards its original position; of course such a motion cannot occur in nature, where neither a force nor a velocity ever is actually infinite.

3. Analytically the problem may be treated separately by means of the equation $\frac{d^{2} x}{d t^{2}}=X$, which is at once integrable in the form $\left(\frac{d x}{d t}\right)^{2}=C+2 \int X d x$.

4. The following cases may be mentioned:

Force $\propto$ Dist. The law of motion is well known, being in fact the same as for the cycloidal pendulum.

Force $\propto(\text { Dist. })^{-2},=\frac{\mu}{x^{2}}$, which is the case above alluded to.

Assuming that the body falls from rest at a distance $a$, we have

$$
x=\alpha(1-\cos \phi),
$$

where, if $n=\frac{a^{\frac{8}{2}}}{\sqrt{\mu}}, \phi$ is given in terms of the time by means of the equation

$$
n t=\phi-\sin \phi .
$$


If the body had initially a small transverse velocity, the motion would be in a very eccentric ellipse, and the formulæ are in fact the limiting form of those for elliptic motion.

5. There are various laws of force for which the motion may be determined. In particular it can be determined by means of Elliptic Integrals, in the case of a body attracted to two centres, force $\propto(\text { dist. })^{-2}$ : see Legendre, Exercices de Cal. Intég., t. II. pp. 502-512, and Théorie des Fonct. Ellip., t. I. pp. 531-538.

Central Forces, Article Nos. 6 to 26.

6. The theory of the motion of a body under the action of a given central force was first established in the Principia, Book I. \$ 2 and 3: viz. Prop. I. the areas are proportional to the times, that is (using the ordinary analytical notation), $r^{2} d \theta=h d t$, and Prop. VI. Cor. $3, P \propto \frac{1}{S Y^{2} \cdot P V},=h^{2} u^{2}\left(\frac{d^{2} u}{d \theta^{2}}+u\right)$, so that

$$
\frac{d^{2} u}{d \theta^{2}}+u-\frac{P}{h^{2} u^{2}}=0
$$

7. It is to be noticed that, given the orbit, the law of force is at once determined; and $\S 2$ contains several instances of such determination; thus,

Prop. VII. If a body revolve in a circle, the law of force to a point $S$ is force $\propto \frac{1}{S P^{2} \cdot P V^{3}}(P$ the body, $P V$ the chord through $S)$.

Prop. IX. If a body move in a logarithmic spiral, force $\propto$ (dist. $)^{-3}$.

Prop. X. If a body move in an ellipse, force to centre $\propto$ dist., and as a particular case, if the body move in a parabola under the action of a force parallel to the axis, the force is constant. The particular case of motion in a parabola had been obtained by Galileo.

And $\S 3$, Props. XI. XII. XIII. If a body move in an ellipse, hyperbola, or parabola under the action of a force tending to the focus, force $\propto$ (dist. $)^{-2}$.

8. But Newton had no direct method of solving the inverse problem (which depends on the solution of the differential equation), "Given the force to find the orbit." Thus force $\propto$ (dist. $)^{-2}$, after it has been shown that an ellipse, a hyperbola, and a parabola may each of them be described under the action of such a force, the remainder of the solution consists in showing that, given the initial circumstances of the motion, a conic section (ellipse, parabola, or hyperbola, as the case may be) can be constructed, passing through the point of projection, having its tangent in the direction of the initial motion, and such that the velocity of the body describing the conic section under the action of the given central force is equal to the velocity of projection; which being so, the orbit will be the conic section so constructed. This is what is done, Prop. XVII.; it may be observed that the latus rectum is constructed not very elegantly by means of the latus rectum of an auxiliary orbit. 
9. A more elegant construction was obtained by Cotes (see the Harmonia Mensurarum, pp. 103-105, and demonstration from the author's papers in the Notes by R. Smith, pp. 124, 125), depending on the position of a point $C$, such that the velocity acquired in falling under the action of the central force from $C$ directly or through infinity $\left.{ }^{1}\right)$ to $P$ the point of projection, is equal to the given velocity of projection.

10. But Newton's original construction is now usually replaced by a construction which employs the space due to the velocity of projection considered as produced by a constant force equal to the central force at the point of projection.

11. $\S 9$ of Book I. relates to revolving orbits, viz., it is shown that a body may be made to move in an orbit revolving round the centre of force, by adding to the central force required to make the body move in the same orbit at rest, a force $\propto\left(\right.$ dist. $^{-3}$. This appears very readily by means of the differential equation (antè, No. 6), viz. writing therein $P+c u^{3}$ for $P$, and then $\theta^{\prime}, h^{\prime}$ in the place of $\theta \sqrt{1-\frac{c}{h^{2}}}, h \sqrt{1-\frac{c}{h^{2}}}$ respectively, the equation retains its original form, with $\theta^{\prime}, h^{\prime}$ in the place of $\theta, h$ respectively.

12. It may be remarked that when the original central force vanishes, the fixed orbit is a right line (not passing through the centre of force). It thus appears by $\S 9$ that the curve $u=A \cos (n \theta+B)$ may be described under the action of a force $\propto(\text { dist. })^{-3}$. A proposition in $\S 2$, already referred to, shows that a logarithmic spiral may be described under the action of such a force.

13. But the case of a force $\propto(\text { dist. })^{-3}$ was first completely discussed by Cotes in

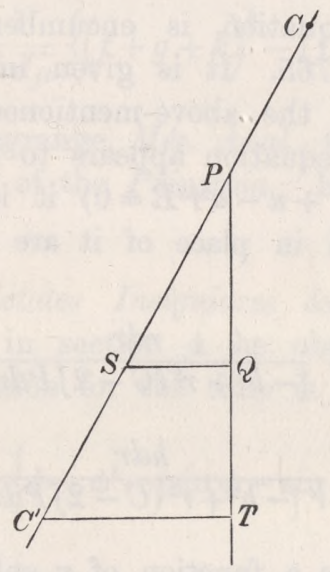

the Harmonia Mensurarum, pp. 31-35, 98-104, and Notes, pp. 117-173. There are in all five cases, according as the velocity of projection is

1. Less than that acquired in falling from infinity, or say equal to that acquired in falling from a point $C$ to $P$, the point of projection.

${ }^{1}$ In the second case $C$ lies on the radius vector produced beyond the centre, and the body is supposed to fall from rest at $C$ (under the action of the central force considered as repulsive) to infinity, and then from the opposite infinity (with an initial velocity equal to the velocity so acquired) to $P$. 
2. Equal to that acquired in falling from infinity.

$3,4,5$. Greater than that acquired in falling from infinity, or say equal to that acquired in falling from a point $C^{\prime}$, through infinity, to $P$; viz. $P Q$ being the direction of projection, and $S Q, C^{\prime \prime} T$ perpendiculars thereon from $S$ and $C^{\prime}$ respectively,
3. $S Q<T Q$;
4. $S Q=T Q$;
5. $S Q>T Q$;

the equations of the orbits being

1. $u=A e^{m \theta}+B e^{-m \theta}, A$ and $B$ same sign, so that rad. vector is never infinite.

2. $u=A e^{m \theta}$ or $B e^{-m \theta}$, logarithmic spiral.

3. $u=A e^{m \theta}+B e^{-m \theta}, A$ and $B$ opposite signs, so that rad. vector becomes infinite.

4. $u=A \theta+B, m=0$, reciprocal spiral.

5. $u=A \cos (n \theta+B), m=n \sqrt{-1}$.

14. The before-mentioned equation,

$$
\frac{d^{2} u}{d \theta^{2}}+u-\frac{P}{h^{2} u^{2}}=0
$$

is in effect given (but the equation is encumbered with a tangential force) in Clairaut's Théorie de la Lune, 1765. It is given in its actual form, and extensively used (in particular for obtaining the above-mentioned equations for Cotes' spirals) in Whewell's Dynamics, 1823. The equation appears to be but little known to continental writers, and (under the form $u^{\prime \prime}+u-\alpha^{2} r^{2} R=0$ ) it is given as new by Schellbach as late as 1853. The formulæ used in place of it are those which give $t$ and $\theta$ each of them in terms of $r$; viz.

$$
\begin{aligned}
d t & =\frac{r d r}{\left\{-h^{2}+r^{2}\left(C-2 \int P d r\right)\right\}^{\frac{1}{2}}}, \\
d \theta & =\frac{h d r}{r\left\{-h^{2}+r^{2}\left(C-2 \int P d r\right)\right\}^{\frac{1}{2}}},
\end{aligned}
$$

which, however, assume that $P$ is a function of $r$ only.

15. Force $\propto(\text { dist. })^{-2}$. The law of motion in the conic sections is implicitly given by Newton's theorem for the equable description of the areas. For the parabola, if $\alpha$ denote the pericentric distance, and $f$ the angle from pericentre or true anomaly, we have

$$
t=\frac{\alpha^{\frac{3}{2}} \sqrt{2}}{\sqrt{\mu}}\left(\tan \frac{1}{2} f+\frac{1}{3} \tan ^{3} \frac{1}{2} f\right)
$$


For the ellipse we have an angle $g$, the mean anomaly varying directly as the time $\left(g=n t\right.$ if $\left.n=\frac{\sqrt{\mu}}{a^{\frac{3}{2}}}\right)$; an auxiliary angle $u$, the eccentric anomaly, connected with $g$ by the equation

$$
g=u-e \sin u
$$

and then the radius vector $r$ and the true anomaly $f$ are given in terms of $u$ by the equations $r=a(1-e \cos u)$, and

$\cos f=\frac{\cos u-e}{1-e \cos u}, \quad \sin f=\frac{\sqrt{1-e^{2}} \sin u}{1-e \cos u}$, and therefore $\tan \frac{1}{2} f=\sqrt{\frac{1+e}{1-e}} \tan \frac{1}{2} u$.

16. It is very convenient to have a notation for $\frac{r}{a}$ and $f$ considered as functions of $e, g$, and I have elsewhere proposed to write

$$
r=a \text { elqr }(e, g), f=\operatorname{elta}(e, g),
$$

read elqr elliptic quotient radius, and elta elliptic true anomaly.

17. The formulæ for the hyperbola correspond to those for the ellipse, but they contain exponential in the place of circular functions (see post, Elliptic Motion).

18. Euler, in the memoir "Determinatio Orbitæ Cometæ Anni 1742," (1743), p. 16 et seq., obtained an expression for the time of describing a parabolic arc in terms of the radius vectors and the chord; viz. these being $f, g$, and $k$, the expression is

$$
\text { Time }=\frac{1}{6 \sqrt{\mu}}\left\{(f+g+k)^{\frac{3}{2}}-(f+g-k)^{\frac{8}{2}}\right\},
$$

which, however, as remarked by Lagrange, Méc. Anal., t. II. (3rd edit. p. 28), is deducible from Lemma X. of the third book of the Principia. But the theorem in its actual form is due to Euler.

19. Lambert, in the Proprietates Insigniores \&c. (1761), Theorem VII. Cor. 2, obtained the same theorem, and in section 4 he obtained the corresponding theorem for elliptic motion; viz. the expression for the time is

if

$$
=\frac{a^{\frac{3}{2}}}{\sqrt{\mu}}\left\{\phi-\phi^{\prime}-\left(\sin \phi-\sin \phi^{\prime}\right)\right\},
$$

$$
\sin \frac{1}{2} \phi=\frac{1}{2} \sqrt{\frac{f+g+k}{a}}, \quad \sin \frac{1}{2} \phi^{\prime}=\frac{1}{2} \sqrt{\frac{f+g-k}{a}} .
$$

The form of the formula is, it will be observed, similar to that for motion in a straight line (antè, No. 4), and in fact the motion in the ellipse is, by an ingenious geometrical transformation, made to depend upon that in the straight line. The geometrical theorems upon which the transformation depends are stated, Cayley "On Lambert's Theorem \&c." (1861). 
20. The theorem was also obtained by Lagrange in the memoir "Recherches \&c." (1767) as a corollary to his solution of the problem of two centres; viz. upon making the attractive force of one of the centres equal to zero, and assuming that such centre is situate on the curve, the expression for the time presents itself in the form given by Lambert's theorem.

21. Two other demonstrations of the theorem are given by Lagrange in the memoir "Sur une manière particulière d'exprimer le temps \&c." (1778), reproduced in Note V. of the second volume of the last edition (Bertrand's) of the Mécanique Analytique. As M. Bertrand remarks, these demonstrations are very complete, very elegant, and very natural, assuming that the theorem is known beforehand.

Demonstrations were also given by Gauss, "Theoria Motus" (1809), p. 119 et seq.; Pagani, "Démonstration d'un théorème \&c." (1834); and (in connexion with Hamilton's Principal Function) by Sir W. R. Hamilton, "On a General Method \&c." (1834), p. 282 ; Jacobi, "Zur Theorie \&c." (1837), p. 122; Cayley, "Note on the Theory of Elliptic Motion" (1856).

22. Connected with the problem of central forces, we have Sir W. R. Hamilton's "Hodograph," which in the paper (Proc. R. Irish Acad. 1847) is defined, and the fundamental properties thereof are stated; viz. if in an orbit round a centre of force there be taken on the perpendicular from the centre on the tangent at each point, a length equal to the velocity at that point of the orbit, the extremities of these lengths will trace out a curve which is the hodograph. As the product of the velocity into the perpendicular on the tangent is equal to twice the area swept out in a unit of time $(v p=h)$, the hodograph is the reciprocal polar of the orbit with respect to a circle described about the centre of force, radius $=\sqrt{h}$. Whence also the tangent at any point of the hodograph is perpendicular to the radius vector through the corresponding point of the orbit, and the product of the perpendicular on the tangent into the corresponding radius vector is $=h$.

If force $\propto(\text { dist. })^{-2}$, the hodograph, quà reciprocal polar of a conic section with respect to a circle described about the focus, is a circle,

23. The following theorem is also given without demonstration; viz. if two circular hodographs, which have a common chord passing or tending through a common centre of force, be both cut at right angles by a third circle, the times of hodographically describing the intercepted arcs (that is, the times of describing the corresponding elliptic arcs) will be equal.

24. Droop, "On the Isochronism \&c." (1856), shows geometrically that the lastmentioned property is equivalent to Lambert's theorem; and an analytical demonstration is also given, Cayley, "A demonstration of Sir W. R. Hamilton's Theorem \&c." (1857). See also Sir W. R. Hamilton's Lectures on Quaternions (1853), p. 614.

25. The laws of central force which have been thus far referred to are force $\propto r, \propto \frac{1}{r^{2}}, \propto \frac{1}{r^{3}} ;$ and it has been seen that the case of a force $P+\frac{C}{r^{3}}$ depends upon that 
of a force $P$, so that the motions for the forces $A r+\frac{C}{r^{3}}$ and $\frac{B}{r^{2}}+\frac{C}{r^{3}}$ are deducible from those for the forces $A r$ and $\frac{B}{r^{2}}$ respectively. Some other laws of force, e.g. $\frac{A}{r^{2}} \pm B r$, $\frac{A}{r^{2}}+\frac{B}{r^{3}}+\frac{C}{r^{4}}+\frac{D}{r^{5}}$, are considered by Legendre, "Théorie des Fonctions Elliptiques" (1825), being such as lead to results expressible by elliptic integrals, and also the law $\frac{M}{r}$, for which the result involves a peculiar logarithmic integral. But the most elaborate examination of the different cases in which the solution can be worked out by elliptic integrals or otherwise is given in Stader's memoir "De Orbitis \&c." (1852), where the investigation is conducted by means of the formulæ which give $t$ and $\theta$ in terms of $r$ (antè, No. 14).

26. In speaking of a central force, it is for the most part implied that the force is a function of the distance: for some problems in which this is not the case, see post, Miscellaneous Problems, Nos. 86 and 87.

It is to be noticed that, although the problem of central forces may be (as it has so far been) considered as a problem in plano (viz. the plane of the motion has been made the plane of reference), yet that it is also interesting to consider it as a problem in space; in fact, in this case the integrals, though of course involved in those which belong to the plane problem, present themselves under very distinct forms, and afford interesting applications of the theory of canonical integrals, of the derivation of the successive integrals by Poisson's method, and of other general dynamical theories. Moreover, in the lunar and planetary theories, the problem must of necessity be so treated. Without going into any details on this point, I will refer to Bertrand's memoir, "Sur les Équations différentielles de la Mécanique" (1852), Donkin's memoir “On a Class of Differential Equations \&c." (1855), and Jacobi's posthumous memoir, "Nova Methodus \&c." (1862).

Elliptic Motion, Article Nos. 27-40.

27. The question of the development of the true anomaly in terms of the mean anomaly (Kepler's problem), and of the other developments which present themselves in the theory of elliptic motion, is one that has very much occupied the attention of geometers. The formulæ on which it depends are mentioned antè, No. 15; they involve as an auxiliary quantity the eccentric anomaly $u$.

28. Consider first the equation

$$
g=u-e \sin u
$$

which connects the mean anomaly $g$ with the eccentric anomaly $u$.

Any function of $u$, and in particular $u$ itself, and the functions ${ }_{\sin }^{\cos } n u$ may be expanded in terms of $g$ by means of Lagrange's theorem (Lagrange, Mém. de Berlin, 1768-1769, “Théorie des Fonctions," chap. 16, and "Traité de la Résolution des Équations Numériques," Note 11).

C. IV. 
29. Considering next the equation

$$
\tan \frac{1}{2} f=\sqrt{\frac{1+e}{1-e}} \tan \frac{1}{2} u
$$

which gives the true anomaly in terms of the eccentric anomaly, then, by replacing the circular functions by their exponential values (a process employed by Lagrange, Mém. de Berlin, 1776), $f$ can be expressed in terms of $u$; viz. the result is

$$
f=u+2 \lambda \sin u+2 \lambda^{2} \cdot \frac{1}{2} \sin 2 u+2 \lambda^{3} \cdot \frac{1}{3} \sin 3 u+\& c .,
$$

where $\lambda=\frac{1-\sqrt{1-e^{2}}}{e}\left(=\frac{e}{1+\sqrt{1-e^{2}}}\right)$. Hence if $u, \sin u, \sin 2 u, \& c$. are expressed in terms of the mean anomaly, $f$ will be obtained in the form $f=g+$ a series of multiple sines of $g$, the coefficients of the different terms being given in the first instance as functions of $e$ and $\lambda$; and to complete the development $\lambda$ and its powers have to be developed in powers of $e$. The solution is carried thus far in the Mécanique Analytique (1788), and in the Mécanique Celeste (1799).

30. We have next Bessel's investigations in the Berlin Memoirs for 1816, 1818, and 1824, and which are carried on mainly by means of the integral

$$
2 \pi I_{k}^{h}=\int_{0}^{2 \pi} \cos (h u-k \sin u) d u,
$$

and various properties are there obtained and applications made of this important transcendant.

31. Relating to this integral we have Jacobi's memoir, "Formulæ transformationis \&c." (1836), Liouville, "Sur l'intégrale $\int_{0}^{\pi} \cos i(u-x \sin u) d u$ " (1841), and Hansen's "Ermittelung der absoluten Störungen" (1843); the-researches of Poisson in the Connaissance des Temps for 1825 and 1836 are closely connected with those of Bessel.

32. A very elegant formula, giving the actual expression of the coefficients considered as functions of $e$ and $\lambda$, is given by Greatheed in the paper "Investigation of the General Term \&c." (1838); viz. this is

$$
f=g+2 \Sigma \lambda^{r}\left\{\epsilon^{\operatorname{tr}\left(\lambda+\lambda^{-1}\right)}+\lambda^{-r} \epsilon^{-\frac{1}{2}\left(\lambda+\lambda^{-1}\right.}\right\} \frac{\sin r g}{r},
$$

where, after developing in powers of $\lambda$, the negative powers of $\lambda$ must be rejected, and the term independent of $\lambda$ divided by 2 . This result is extended to other functions of $f$, Cayley "On certain Expansions \&c." (1842).

33. An expression for the coefficient of the general term as a function of $e$ only is obtained, Lefort, "Expression Numérique \&c." (1846). The expression, which, from the nature of the case, is a very complicated one, is obtained by means of Bessel's integral. This is an indirect process which really comes to the combination of the developments of $f$ in terms of $u$, and $u$ in terms of $g$; and an equivalent result is obtained directly in this manner, Creedy, "General and Practical Solution \&c." (1855). 
34. We have also on the subject of these developments the very valuable and interesting researches of Hansen, contained in his Fundamenta Nova \&c. (1838), in the memoir "Ermittelung der absoluten Störungen \&c." (1845), and in particular in the memoir "Entwickelung des Products \&c." (1853).

35. But the expression for the coefficient of the general term ${ }_{\sin }^{\cos } r g$ in any of these expansions is so complicated that it was desirable to have for the coefficients corresponding to the values $r=0,1,2,3, \ldots$ the finally reduced expressions in which the coefficient of each power of $e$ is given as a numerical fraction. Such formulæ for the development of $\left(\frac{r}{a}-1\right)^{m} \cos j f$, where $j$ is a general symbol, the expansion being carried as far as $e^{7}$, were given, Leverrier, Annales de l'Observatoire de Paris, t. I. (1855).

36. And starting from these I deduced the results given in my "Tables of the Developments \&c." (1861); viz. these tables give $\left(x=\frac{r}{a}-1\right)$,

$$
\begin{aligned}
& \left(x^{1}, \ldots . x^{7}\right), \\
& \left(x^{0}, x^{1}, \ldots x^{7}\right) \sin _{\cos }^{\cos } j, j=1 \text { to } j=7, \\
& \left(\left(\frac{r}{a}\right)^{4},\left(\frac{r}{a}\right)^{3} \ldots \log \frac{r}{a},\left(\frac{r}{a}\right)^{-1}, \ldots\left(\frac{r}{a}\right)^{-5}\right), \\
& \left(\left(\frac{r}{a}\right)^{4}, \ldots\left(\frac{r}{a}\right)^{1},\left(\frac{r}{a}\right)^{-1}, \ldots\left(\frac{r}{a}\right)^{-5}\right)_{\sin ^{\cos } j f, j=1 \text { to } j=7,}
\end{aligned}
$$

all carried to $e^{7}$.

37. The true anomaly $f$ has been repeatedly calculated to a much greater extent, in particular by Schubert (Ast. Théorique, St Pét. 1822), as far as $e^{20}$. The expression for $\frac{r}{a}$ as far as $e^{13}$ is given in the same work, and that for $\log \frac{r}{a}$ as far as $e^{9}$ was calculated by Oriani, see Introd. to Delambre's Tables du Soleil, Paris (1806).

38. It may be remarked that when the motion of a body is referred to a plane which is not the plane of the elliptic orbit, then we have questions of development similar in some measure to those which regard the motion in the orbit; if, for instance, $z$ be the distance from node, $\phi$ the inclination, and $x$ the reduced distance from node, then $\cos z=\cos \phi \cos x$, from which we may derive $z=x+$ series of multiple sines of $x$. And there are, moreover, the questions connected with the development of the reciprocal distance of two particles-say $\left(a^{2}+a^{\prime 2}-2 a a^{\prime} \cos \theta\right)^{-\frac{1}{2}}$-which present themselves in the planetary theory; but this last is a wide subject, which I do not here enter upon. I will, however, just refer to Hansen's memoir, "Ueber die Entwickelung der negativen und ungeraden Potenzen \&c." (1854). 
39. The question of the convergence of the series is treated in Laplace's memoir of 1823 , where he shows that in the series which express $r$ and $f$ in multiple cosines or sines of $g$, the coefficient of a term $\frac{\cos }{\sin } i g$, where $i$ is very great, is at most equal in absolute value to a quantity of the form $\frac{A}{i \sqrt{ } i}\left(\frac{e}{\lambda}\right)^{i}, A$ and $\lambda$ being finite quantities independent of $i$, whence he concludes that, in order to the convergency of the series, the limiting value of the eccentricity is $e=\lambda$, the numerical value being $e=0.66195$.

40. The following important theorem was established by Cauchy, as part of a theory of the convergence of series in general; viz. so long as $e$ is less than 0.6627432 , which is the least modulus of $e$ for which the equations

$$
\frac{\pi}{2}=u-e \sin u, \quad 1-e \cos u=0,
$$

can be satisfied, the development of the true anomaly and other developments in the theory of elliptic motion will be convergent. This was first given in the "Mémoire sur la Mécanique Céleste," read at Turin in 1831, but it is reproduced in the memoir "Considérations nouvelles sur les suites \&c.," Mém. d’Anal. et de Phys. Math. t. I. (1840); and see also the memoirs in Liouville's Journal by Puiseux, and his Note i. to vol. II. of the 3rd ed. of the Mécanique Analytique (1855). There are on this subject, and on subjects connected with it, several papers by Cauchy in the Comptes Rendus, 1840 et seq., which need not be particularly referred to.

The Problem of two Centres, Article Nos. 41 to 64.

41. The original problem is that of the motion of a body acted upon by forces tending to two centres, and varying inversely as the squares of the distances; but, as will be noticed, the solutions apply with but little variation to more general laws of force.

42. It may be convenient to notice that the coordinates made use of (in the several solutions) for determining the position of the body, are either the sum and difference of the two radius vectors, or else quantities which are respectively functions of the sum and the difference of these radius vectors ${ }^{(1)}$. If the plane of the motion is not given, then there is a third coordinate, which is the inclination of the plane through the body and the two centres to a fixed plane through the two centres, or say the azimuth of the axial plane, or simply the azimuth.

${ }^{1}$ If $v, u$ are the distances of the body $P$ from the centres $A$ and $B, a$ the distance $A B, \zeta, \eta$ the angles at $A$ and $B$ respectively, and $p=\tan \frac{1}{2} \zeta \tan \frac{1}{2} \eta, q=\tan \frac{1}{2} \zeta \div \tan \frac{1}{2} \eta$, then, as may be shown without difficulty, $v+u=a \frac{1+p}{1-p}, v-u=a \frac{1-q}{1+q}$, so that $p$ and $q$ are functions of $v+u$ and $v-u$ respectively; these quantities $p$ and $q$ are Euler's original coordinates. 
39. The question of the convergence of the series is treated in Laplace's memoir of 1823 , where he shows that in the series which express $r$ and $f$ in multiple cosines or sines of $g$, the coefficient of a term $\frac{\cos }{\sin } i g$, where $i$ is very great, is at most equal in absolute value to a quantity of the form $\frac{A}{i \sqrt{ } i}\left(\frac{e}{\lambda}\right)^{i}, A$ and $\lambda$ being finite quantities independent of $i$, whence he concludes that, in order to the convergency of the series, the limiting value of the eccentricity is $e=\lambda$, the numerical value being $e=0.66195$.

40. The following important theorem was established by Cauchy, as part of a theory of the convergence of series in general; viz. so long as $e$ is less than 0.6627432, which is the least modulus of $e$ for which the equations

$$
\frac{\pi}{2}=u-e \sin u, \quad 1-e \cos u=0
$$

can be satisfied, the development of the true anomaly and other developments in the theory of elliptic motion will be convergent. This was first given in the "Mémoire sur la Mécanique Céleste," read at Turin in 1831, but it is reproduced in the memoir "Considérations nouvelles sur les suites \&c.," Mém. d'Anal. et de Phys. Math. t. I. (1840); and see also the memoirs in Liouville's Journal by Puiseux, and his Note i. to vol. II. of the 3rd ed. of the Mécanique Analytique (1855). There are on this subject, and on subjects connected with it, several papers by Cauchy in the Comptes Rendus, 1840 et seq., which need not be particularly referred to.

The Problem of two Centres, Article Nos. 41 to 64 .

41. The original problem is that of the motion of a body acted upon by forces tending to two centres, and varying inversely as the squares of the distances; but, as will be noticed, the solutions apply with but little variation to more general laws of force.

42. It may be convenient to notice that the coordinates made use of (in the several solutions) for determining the position of the body, are either the sum and difference of the two radius vectors, or else quantities which are respectively functions of the sum and the difference of these radius vectors ${ }^{1}{ }^{1}$. If the plane of the motion is not given, then there is a third coordinate, which is the inclination of the plane through the body and the two centres to a fixed plane through the two centres, or say the azimuth of the axial plane, or simply the azimuth.

\footnotetext{
${ }^{1}$ If $v, u$ are the distances of the body $P$ from the centres $A$ and $B, a$ the distance $A B, \zeta, \eta$ the angles at $A$ and $B$ respectively, and $p=\tan \frac{1}{2} \zeta \tan \frac{1}{2} \eta, q=\tan \frac{1}{2} \zeta \div \tan \frac{1}{2} \eta$, then, as may be shown without difficulty, $v+u=a \frac{1+p}{1-p}, v-u=a \frac{1-q}{1+q}$, so that $p$ and $q$ are functions of $v+u$ and $v-u$ respectively; these quantities $p$ and $q$ are Euler's original coordinates.
} 
43. Calling the first-mentioned two coordinates $r$ and $s$, and the azimuth $\psi$, the solution of the problem leads ultimately to equations of the form

$$
\frac{d r}{\sqrt{R}}=\frac{d s}{\sqrt{\bar{S}}}, \quad d t=\frac{\lambda d r}{\sqrt{R}}+\frac{\mu d s}{\sqrt{\bar{S}}}, \quad \delta \psi=\frac{\rho d r}{\sqrt{R}}+\frac{\sigma d s}{\sqrt{S}}
$$

where $R$ and $S$ are rational and integral functions (of the third or fourth degree, in the case of forces varying as (dist.) ${ }^{-2}$ ) of $r, s$ respectively (but they are not in general the same functions of $r, s$ respectively); $\lambda$ and $\rho$ are simple rational functions of $r$, and $\mu$ and $\sigma$ simple rational functions of $s$; so that the equations give by quadratures, the first of them the curve described in the axial plane, the second the position of the body in this curve at a given time, and the third of them the position of the axial plane. In the ordinary case, where $R$ and $S$ are each of them of the third or the fourth order, the quadratures depend on elliptic integrals $\left(^{1}\right)$; but on account of the presence in the formulæ of the two distinct radicals $\sqrt{R}, \sqrt{S}$, it would appear that the solution is not susceptible of an ulterior development by means of elliptic and Jacobian functions $\left(^{1}\right)$ similar to those obtained in the problems of Rotation and the Spherical Pendulum.

44. It has just been noticed that when $R, S$ are each of them of the fourth order, the quadratures depend on elliptic integrals; in the particular cases in which the relation between $r, s$ is of the form $\frac{m d r}{\sqrt{R}}=\frac{n d s}{\sqrt{S}}, R$ and $S$ being the same functions of $r, s$ respectively, and $m$ and $n$ being integers (or more generally for other relations between the forms of $R, S$ given by the theory of elliptic integrals), the equation admits of algebraical integration; but as the relations in question do not in general hold good, the theory of the algebraical integration of the equations plays only a secondary part in the solution of the problem. It is, however, proper to remark that Euler, when he wrote his first two memoirs "On the Problem of the two Centres" (post, Nos. 45 and 46), had already discovered and was acquainted with the theory of the algebraic integration of the equation $\frac{m d r}{\sqrt{ } R}=\frac{n d s}{\sqrt{ } S}(R, S, m, n$, ut suprà), although his memoir, "Integratio æquationis

$$
\frac{d x}{\sqrt{A+B x+C x^{2}+D x^{3}+E x^{4}}}=\frac{d y}{\sqrt{A+B y+C y^{2}+D y^{3}+E y^{4}}}
$$

N. Comm. Petrop. t. XII. 1766-1767?, bears in fact a somewhat later date.

45. Having made these preliminary remarks, I come to the history of the problem.

It is I think clear that Euler's earliest memoir is the one "De Motu Corporis \&c." in the Petersburg Memoirs for 1764 (printed 1766). In this memoir the forces vary

1 The elliptic integrals are Legendre's functions $F, E, \Pi$; the elliptic and Jacobian functions are sinam, cosam, $\Delta$ am, and the higher transcendants, $\theta, H$. 
where

$$
\begin{aligned}
& P=(A+B+D) p+2 E p^{2}+(-A-B+D) p^{3} \\
& Q=(-A+B-D) q+2 E q^{2}+(A-B-D) q^{3}
\end{aligned}
$$

so that $P$ and $Q$ are cubic functions (not the same functions) of $p$ and $q$ respectively and the equation for the time is found to be

$$
\frac{d t \sqrt{2 g}}{a \sqrt{a}}=\frac{p d p}{(1-p)^{2} \sqrt{P}}+\frac{q d q}{(1+q)^{2} \sqrt{Q}},
$$

which are the formulæ for the solution of the problem, as obtained in Euler's first and second memoirs.

47. In his third memoir, viz. that "De Motu Corporis \&c." in the Petersburg Memoirs for 1765 (printed 1767), Euler considers the body as moving in space, the forces being as before as (dist.) ${ }^{-2}$. Assuming that the coordinates $y, z$ are in the plane perpendicular to the axis, there is in this case the equation of areas $y \frac{d z}{d t}-z \frac{d y}{d t}=$ const.; and writing $y=y^{\prime} \sin \psi, z=y^{\prime} \cos \psi$, that is, $y^{\prime}=\sqrt{y^{2}+z^{2}}$, and $\psi$ the azimuth, the integral equations for the motion in the variable plane (coordinates $x, y^{\prime}$ ) are not materially different in form from those which belong to the motion in a fixed plane, coordinates $x, y$ (see post, No. 56, Jacobi); and the last-mentioned equation, which reduces itself to the form $y^{\prime 2} \frac{d \psi}{d t}=$ const., gives at once $d \psi$ in a form such as that above alluded to (antè, No. 43), and therefore $\psi$ by quadratures. The variables employed by Euler in the memoir in question are

$$
v+u, \quad v-u,(\text { say } r, s), \text { and } \psi,
$$

$v, u$ being, as above, the distances from the two centres, and $\psi$ the azimuth of the axial plane. The functions of $r, s$ under the radical signs are of the fourth order; this is so, with these variables, even if the motion is in a fixed plane; but this is no disadvantage, since, as is well known, the case of a quartic radical is not really more complicated than that of a cubic radical, the two forms being immediately convertible the one into the other.

48. Lagrange's first memoir (Turin Memoirs, 1766-1769) refers to Euler's three memoirs, but the author mentions that it was composed in 1767 without the knowledge of Euler's third memoir. The coordinates ultimately made use of are $v+u, v-u$, (say $r, s$ ), and $\psi$, the same as in Euler's third memoir, and the results consequently present themselves in the like form.

49. If the attractive force of one of the centres is taken equal to zero, then the position of such centre is arbitrary, and it may be assumed that the centre lies on the curve, which is in this case an ellipse (conic section); the expression of the time presents itself as a function of the focal radius vectors and the chord of the arc described; which, as remarked, antè, No. 20, leads to Lambert's theorem for elliptic motion. 
50. The case presents itself of an ellipse or hyperbola described under the action of the two forces, viz. the equation $\frac{d r}{\sqrt{R}}=\frac{d s}{\sqrt{S}}$ will be satisfied by $r-\alpha=0$, if $r-\alpha$ be a double factor of $R$, or by $s-\beta=0$, if $s-\beta$ be a double factor of $S$, a case which is also considered in the Mécanique Analytique; and see in regard to the analytical theory, t. II. 3rd ed. Note III. by M. Serret, and "Thèse," Liouv. 1848. It is remarked by M. Bonnet, Note IV. and Liouv. t. Ix. p. 113, (1844), that the result is a mere corollary of a general theorem, which is in effect as follows, viz. if a particle under the separate actions of the forces $F, F^{\prime}, \ldots$ starting in each case from the same point in the same direction but with the initial velocities $v, v^{\prime}$; \&c. respectively, describe the same curve, then such curve will also be described under the conjoint action of all the forces, provided the body start from the same point in the same direction, with the initial velocity $V=\sqrt{v^{2}+v^{\prime 2}+\ldots}$.

51. Lagrange's second memoir (same volume of the Turin Memoirs) contains an exceedingly interesting discussion as to the laws of force for which the problem can be solved. Writing $U, V, u, v$ in the place of Lagrange's $P, Q, p$, $q$, the equations of motion are

where

$$
\begin{aligned}
& \frac{d^{2} x}{d t^{2}}+\frac{(x-a) U}{u}+\frac{(x-\alpha) V}{v}=0 \\
& \frac{d^{2} y}{d t^{2}}+\frac{(y-b) U}{u}+\frac{(y-\beta) V}{v}=0 \\
& \frac{d^{2} z}{d t^{2}}+\frac{(z-c) U}{u}+\frac{(z-\gamma) V}{v}=0
\end{aligned}
$$

$$
\begin{aligned}
& u=\sqrt{(x-a)^{2}+(y-b)^{2}+(z-c)^{2},} \\
& v=\sqrt{(x-\alpha)^{2}+(y-\beta)^{2}+(z-\gamma)^{2}},
\end{aligned}
$$

and putting also $f\left(=\sqrt{(a-\alpha)^{2}+(b-\beta)^{2}+(z-\gamma)^{2}}\right)$ the distance of the centres, and then $u^{2}=f^{2} x, v^{2}=f^{2} y, \frac{U}{u}=X, \frac{V}{v}=Y(x, y$ are of course not to be confounded with the coordinates originally so represented), Lagrange obtains the equations

$$
\begin{aligned}
& \frac{1}{2} \frac{d^{2} x}{d t^{2}}+X x+\frac{(x+y-1) Y}{2}+\int(X d x+Y d y)=0, \\
& \frac{1}{2} \frac{d^{2} y}{d t^{2}}+Y y+\frac{(x+y-1) X}{2}+\int(X d x+Y d y)=0,
\end{aligned}
$$

which he represents by

$$
\begin{aligned}
& \frac{1}{2} \frac{d^{2} x}{d t^{2}}+M=0 \\
& \frac{d^{2} y}{2} \frac{d^{2}}{d t^{2}}+N=0
\end{aligned}
$$

and he then inquires as to the conditions of integrability of these equations, for which purpose he assumes that the equations multiplied by $m d x+n d y$ and $\mu d x+\nu d y$ respectively and added, give an integrable equation. 
52. A case satisfying the required conditions is found to be

$$
X=2 \alpha+\frac{\beta}{x \sqrt{x}}, \quad Y=2 \alpha+\frac{\gamma}{y \sqrt{y}},
$$

or, what is the same thing,

$$
U=2 \alpha u+\frac{\beta f^{3}}{u^{2}}, \quad V=2 \alpha v+\frac{\gamma f^{3}}{v^{2}}
$$

that is, besides the forces $\frac{\beta f^{3}}{u^{2}}, \frac{\gamma f^{3}}{v^{2}}$, which vary as (dist.) $)^{-2}$, there are the forces $2 \alpha u, 2 \alpha v$, varying directly as the distance, and of the same amount at equal distances; or, what is the same thing, there is, besides the forces varying as (dist.) ${ }^{-2}$, a force varying directly as the distance, tending to a third centre midway between the other two, a case which is specially considered in the memoir; it is found that the functions in $r, s$ under the radicals (instead of rising only to the order 4 ) rise in this case to the order 6 .

53. Among other cases are found the following, viz. :

$$
\begin{array}{ll}
1^{\circ} . & U=\alpha u+\frac{7 \lambda}{f^{2}} u^{3}+\frac{5 \lambda}{f^{4}} u^{5}, \\
& V=\alpha v+\frac{7 \lambda}{f^{2}} v^{3}+\frac{5 \lambda}{f^{4}} v^{5} ; \\
2^{\circ} . & U=\alpha u+\frac{\beta}{f^{2}} u^{3}, \\
V & =\delta v+\frac{\epsilon}{f^{2}} v^{3},
\end{array}
$$

where $\beta=\epsilon$, or else $\alpha \epsilon=\beta \delta=2 \beta \epsilon$.

In regard to the subject of this second memoir of Lagrange, see post, Miscellaneous Problems, Liouville's Memoirs, Nos. 100 to 105.

54. In the Mécanique Analytique (1st ed. 1788, and 2nd ed, t. II. 1813), Lagrange in effect reproduces his solution for the above-mentioned law of force (say $U=\frac{\alpha}{u^{2}}+2 \gamma u$, $\left.\left.V=\frac{\beta}{v^{2}}+2 \gamma v\right) .{ }^{1}\right)$ There are even in the third edition a few trifling errors of work to be corrected. The remarks above referred to, as made by Lagrange in his first memoir, are also reproduced (see antè, Nos. 49 and 50).

55. Legendre, Exercices de Calcul Intégral, t. II. (1817), and Théorie des Fonctions Elliptiques, t. I. (1825), uses $p^{2}$ and $q^{2}$ in the place of Euler's $p, q$; the forces are assumed to vary as (dist.) $)^{-2}$, and in consequence of the change Euler's cubic radicals are replaced by quartic radicals involving only even powers of $p$ and $q$.

${ }^{1}$ In the Mécanique Analytique, Lagrange's letters are $r, q$ for the distances $r+q=s, r-q=u$ : the change in the present Report was occasioned by the retention of Euler's variables $p, q$.

C. IV. 
respectively; that is, the radicals are in a form adapted for the transformation to elliptic integrals; in certain cases, however, it becomes necessary to attribute to Legendre's variables $p$ and $q$ imaginary values. The various cases of the motion are elaborately discussed by means of the elliptic integrals; in particular Legendre notices certain cases in which the motion is oscillatory, and which, as he remarks, seem to furnish the first instance of the description by a free particle of only a finite portion of the curve which is analytically the orbit of the particle; there is, however, nothing surprising in this kind of motion, although its existence might easily not have been anticipated.

56. $§ 26$ of Jacobi's memoir "Theoria Novi Multiplicatoris \&c." (1845) is entitled "Motus puncti versus duo centra secundum legem Neutonianum attracti." The equations for the motion in space are by a general theorem given in the memoir "De Motu puncti singularis" (1842), reduced to the case of motion in a plane: viz. if $x, y$ are the coordinates, the centre point of the axis being the origin, and $y$ being at right angles to the axis, and if the distance of the centres is $2 a$; then the only difference is that to the expression for $\frac{d^{2} y}{d t^{2}}$ there is added a term $\frac{a^{2}}{y^{3}}$, which arises from the rotation about the axis. Two integrals are obtained, one the integral of Vis Viva, and the other of them an integral similar to one of those of Euler's or Lagrange's. And then $x^{\prime}, y^{\prime}$ being the differential coefficients of $x, y$ with regard to the time, the remaining equation may be taken to be $y^{\prime} d x-x^{\prime} d y=0$, where $x^{\prime}, y^{\prime}$ are to be expressed as functions of $x, y$ by means of the two given integrals. This being so, the principle of the Ultimate Multiplier $\left(^{1}\right)$ furnishes a multiplier of this differential equation, and the integral is found to be

$$
\int \frac{y^{\prime} d x-x^{\prime} d y}{x y\left(x^{\prime 2}-y^{\prime 2}\right)+\left(a^{2}-x^{2}+y^{2}\right) x^{\prime} y^{\prime}}=\epsilon,
$$

the quantity under the integral sign being a complete differential. To verify $\grave{a}$ posteriori that this is so, Jacobi introduces the auxiliary quantities $\lambda^{\prime}, \lambda^{\prime \prime}$ defined as the roots of the equation $\lambda^{2}+\lambda\left(x^{2}+y^{2}-a^{2}\right)-a^{2} y^{2}=0$, which in fact, if as before $u, v$ are the distances from the centres, leads to

$$
u+v=2 \sqrt{a^{2}-\lambda^{\prime}}, \quad u-v=2 \sqrt{a^{2}-\lambda^{\prime \prime}},
$$

so that $\lambda^{\prime}, \lambda^{\prime \prime}$ are functions of $u+v, u-v$ respectively; and the formulæ, as ultimately expressed in terms of $\lambda^{\prime}, \lambda^{\prime \prime}$, are substantially of the same form with those of Euler and Lagrange.

57. The investigations contained in Liouville's three memoirs "Sur quelques cas particuliers \&c." (1846), find their chief application in the problem of two centres, and by leading in the most direct and natural manner to the general law of force for which the integration is possible, they not only give some important extension of the problem, but they in fact exhibit the problem itself and the preceding solutions of it in their true light. But as they do not relate to this problem exclusively, it will be convenient to consider them separately under the head Miscellaneous Problems.

${ }^{1}$ Explained in Jacobi's memoir "Theoria Novi Multiplicatoris \&c.," Crelle, tt. xxvir., xxvirI., xxIx. 1844-45. 
58. In Serret's "Thèse sur le Mouvement \&c." (1848), the problem is very elegantly worked out according to the principles of Liouville's memoirs as follows: viz. assuming that the expression of the distance between two consecutive positions of the body is

$$
d s^{2}=\lambda\left(m d \mu^{2}+n d \nu^{2}\right)+\lambda^{\prime \prime} d \gamma^{2},
$$

where $m, n$ are functions of $\mu, \nu$ respectively, and if the forces can be represented by means of a force-function $U$, then the motion can be determined, provided only $\lambda, \lambda U, \frac{\lambda^{\prime \prime}}{\lambda}$ are of the forms

$$
\begin{array}{r}
\lambda=\phi \mu-\dot{\Phi} \nu \\
\lambda U=\psi \mu-\Psi \nu, \\
\frac{\lambda}{\lambda^{\prime \prime}}=\varpi \mu-\Pi \nu,
\end{array}
$$

where the functional symbols $\phi, \Phi, \& c$. denote any arbitrary functions whatever.

59. It is then assumed that $\mu, \nu$ are the parameters of the confocal ellipses and hyperbolas situate in the moveable plane through the axis, viz. that we have

$$
\begin{aligned}
& \frac{x^{2}}{\mu^{2}}+\frac{y^{2}}{\mu^{2}-b^{2}}=1, \\
& \frac{x^{2}}{\nu^{2}}-\frac{y^{2}}{b^{2}-\nu^{2}}=1,
\end{aligned}
$$

(the origin is midway between the two centres, $2 b$ being their distance; $\frac{1}{2} \mu, \frac{1}{2} \nu$ are in fact equal to the sum and difference $u+v, u-v$ of the two centres respectively); and that the position of the moveable plane is determined by means of $\gamma$, the inclination to a fixed plane through the axis, or say, as before, its azimuth. In fact, with these values of the coordinates, the expression of $d s^{2}$ is

$$
d s^{2}=\left(\mu^{2}-\nu^{2}\right)\left(\frac{d \mu^{2}}{\mu^{2}-b^{2}}+\frac{d \nu^{2}}{b^{2}-\nu^{2}}\right)+\frac{\left(\mu^{2}-b^{2}\right)\left(b^{2}-\nu^{2}\right)}{b^{2}} d \gamma^{2},
$$

which is of the required form. And moreover if the forces to the two centres vary as (dist. $)^{-2}$, and there is besides a force to the middle point varying as the distance, then

$$
U=\frac{g}{\mu+\nu}+\frac{g^{\prime}}{\mu-\nu}+K\left(\mu^{2}+\nu^{2}-b^{2}\right),
$$

whence (observing that $\lambda=\mu^{2}-\nu^{2}$ ) $\lambda U$ is of the required form. The equations obtained by substituting for $U$ the above value give the ordinary solution of the problem.

60. Liouville's note to the last-mentioned memoir (1848) contains the demonstration of a theorem obtained by a different process in his second memoir, but which is in the present note, starting from Serret's formulæ, demonstrated by the more simple method of the first memoir, viz., it is shown that the motion can be obtained if the two centres, instead of being fixed, revolve about the point midway between them in 
a circle in such manner that the diameter through the two centres always passes through the projection of the body on the plane of the circle. It will be observed that the circular motion of the two centres is neither a uniform nor a given motion, but that they are, as it were, carried along with the moving body.

61. In Desboves's memoir "Sur le Mouvement d'un point matériel \&c." (1848), the author developes the solution of the foregoing problem of moving centres, chiefly by the aid of the method employed in Liouville's second memoir. And he shows also that the methods of Euler and Lagrange for the case of two fixed centres apply with modification to the more complicated problem of the moving centres.

62. The problem of two centres is considered in Bertrand's "Mémoire sur les équations différentielles \&c." (1852), by means of Jacobi's form of the equations of motion, viz., the problem is reduced to a plane one by means of the addition of a force $\propto \frac{1}{y^{3}}$ (antè, No. 56).

63. Cayley's "Note on Lagrange's Solution \&c." (1857) is merely a reproduction of the investigation in the Mécanique Analytique; the object was partly to correct some slight errors of work, and partly to show what were the combinations of the differential equations, which give at once the integrals of the problem.

64. In § II. of Bertrand's "Mémoire sur quelques unes des formes \&c." (1857), the following question is considered, viz., assuming that the dynamical equations

$$
\frac{d^{2} x}{d t^{2}}=\frac{d U}{d x}, \quad \frac{d^{2} y}{d t^{2}}=\frac{d U}{d y},
$$

have an integral of the form

$$
\alpha=P x^{\prime 2}+Q x^{\prime} y^{\prime}+R y^{\prime 2}+S y^{\prime}+T x^{\prime}+K
$$

(where $\alpha$ is the arbitrary constant, and $P, Q, \ldots K$ are functions of $x$ and $y$ ), it is required to find the form of the force-function $U$. It is found that $U$ must satisfy a certain partial differential equation of the second order, the general solution of which is not known; but taking $U$ to be a function of the distance from any fixed point (or rather the sum of any number of such functions), it is shown that the only case in which the differential equations for the motion of a point attracted to a fixed centre of forces have an integral of the form in question is the above-mentioned one of two centres, each attracting according to the inverse square of the distance, and a third centre midway between them, attracting as the distance.

The Spherical Pendulum, Article Nos. 65 to 73.

65. The problem is obviously the same as that of a heavy particle on the surface of a sphere.

I have not ascertained whether the problem was considered by Euler. Lagrange refers to a solution by Clairaut, Mém. de l'Acad., 1735 . 
The question was considered by Lagrange, Méc. Anal., 1st edit. p. 283. The angles which determine the position are $\psi$ the inclination of the string to the horizon, $\phi$ the inclination of the vertical plane through the string to a fixed vertical plane, or say the azimuth. And then forming the equations of motion, two integrals are at once obtained; these are the integrals of Vis Viva, and an integral of areas. And these give equations of the form $d t=$ funct. $(\psi) d \psi, d \phi=$ funct. $(\psi) d \psi$; so that $t, \phi$ are each of them given by a quadrature in terms of $\psi$, which is the point to which the solution is carried. It is noticed that $\psi$ may have a constant value, which is the case of the conical pendulum.

66. In the second edition, t. XI. p. 197 (1815), the solution is reproduced; only, what is obviously more convenient, the angles are taken to be

$$
\begin{aligned}
& \psi \text {, the inclination to the vertical, } \\
& \phi \text {, the azimuth. }
\end{aligned}
$$

It is remarked that $\psi$ will always lie between a greatest value $\alpha$ and a least value $\beta$, and the integrals are transformed by introducing therein instead of $\psi$ the angle $\sigma$, which is such that

$$
\cos \psi=\cos \alpha \sin ^{2} \sigma+\cos \beta \cos ^{2} \sigma,
$$

by which substitution they assume a more elegant form, involving only the radical

$$
\sqrt{1+k^{2}(\cos \beta-\cos \alpha) \cos 2 \sigma},
$$

where $k$ is a constant depending on $\cos \alpha, \cos \beta$; and the integration is effected approximately in the case where $\cos \beta-\cos \alpha$ is small.

M. Bravais has noticed, however, that by reason of some errors in the working out, Lagrange has arrived at an incorrect value for the angle $\Phi$, which is the apsidal angle, or difference of the azimuths for the inclinations $\alpha$ and $\beta$ : see the 3 rd edition (1855), Note VII., where M. Bravais resumes the calculation, and he arrives at the value $\Phi=\frac{1}{2} \pi\left(1+\frac{3}{8} \alpha \beta\right), \alpha$ and $\beta$ being small.

Lagrange considers also the case where the motion takes place in a resisting medium, the resistance varying as velocity squared.

67. A similar solution to Lagrange's, not carried quite so far, is given in Poisson's Mécanique, t. I. pp. 385 et seq. (2nd ed. 1833).

A short paper by Puiseux, "Note sur le Mouvement d'un point matériel sur une sphère" (1842), shows merely that the angle $\Phi$ is $>\frac{1}{2} \pi$.

68. The ulterior development of the solution consists in the effectuation of the integrations by the elliptic and Jacobian functions. It is proper to remark that the dynamical problem the solution whereof by such functions was first fairly worked out, 
is the more difficult one of the rotation of a solid body, as solved by Jacobi (1839), in completion of Rueb's solution (1834), post, Nos. 186 and 197.

69. In relation to the present problem we have Gudermann's memoir "De pendulis sphæricis \&c." (1849), who, however, does not arrive at the actual expressions of the coordinates in terms of the time; and the perusal of the memoir is rendered difficult by the author's peculiar notations for the elliptic functions $\left({ }^{1}\right)$.

70. It would appear that a solution involving the Jacobian functions was obtained by Durège, in a memoir completed in 1849 , but which is still unpublished; see $\S \mathrm{XX}$. of his Theorie der elliptischen Functionen (1861), where the memoir is in part reproduced. It is referred to by Richelot in the Note presently mentioned.

71. We have next Tissot's Thèse de Mécanique, 1852, where the expressions for the variables in terms of the time are completely obtained by means of the Jacobian functions $H, \Theta$, and which appears to be the earliest published one containing a complete solution and discussion of the problem.

72. Richelot, in the Note "Bemerkungen zur Theorie des Raumpendels" (1853), gives also, but without demonstration, the final expressions for the coordinates in terms of the time.

Donkin's memoir "On a Class of Differential Equations \&c." (1855) contains (No. 59) an application to the case of the spherical pendulum.

73. The first part of the memoir by Dumas, "Ueber die Bewegung des Raumpendels, \&c." (1855), comprises a very elegant solution of the problem of the spherical pendulum based upon Jacobi's theorem of the Principal Function (1837), and which is completely developed by the elliptic and Jacobian functions. The latter part of the memoir relates to the effect of the rotation of the Earth; and we thus arrive at the next division of the general subject.

Motion as affected by the Rotation of the Earth, and Relative Motion in general.. Article Nos. 74 to 85.

74. Laplace (Méc. Céleste, Book X. c. 5) investigates the equations for the motion of a terrestrial body, taking account of the rotation of the Earth (and also of the resistance of the air), and he applies them to the determination of the deviations of falling bodies, \&c. He does not, however, apply them to the case of the pendulum.

75. We have also the memoir of Gauss, "Fundamental-gleichungen \&c." (1804): the equations ultimately obtained are similar to those of Poisson. I have not had the opportunity of consulting this memoir.

1 The mere use of $\mathrm{sn}, \mathrm{cn}, \mathrm{dn}$ as an abbreviation of the somewhat cumbrous sinam, cosam, $\Delta \mathrm{am}$ of the Fundamenta Nova is decidedly convenient. 
76. Poisson, in the "Mémoire sur le mouvement des Projectiles \&c." (1838), also obtains the general equations of motion, viz. (omitting terms involving $n^{2}$ ), these may be taken to be

$$
\begin{aligned}
& \frac{d^{2} x}{d t^{2}}=\quad X+2 n\left(\frac{d y}{d t} \sin \beta+\frac{d z}{d t} \cos \beta\right), \\
& \frac{d^{2} y}{d t^{2}}=\quad Y+2 n \frac{d x}{d t} \sin \beta \\
& \frac{d^{2} z}{d t^{2}}=g+Z+2 n \frac{d x}{d t} \cos \beta
\end{aligned}
$$

(see p. 20), where the axes of $x, y, z$ are fixed on the Earth and moveable with it: viz., $z$ is in the direction of gravity; $x, y$ in the directions perpendicular to gravity, viz., $y$ in the plane of the meridian northwards, $x$ westwards; $g$ is the actual force of gravity as affected by the resolved part of the centrifugal force; $\beta$ is the latitude. There are some niceties of definition which are carefully given by Poisson, but which need not be noticed here.

77. Poisson applies his formulæ incidentally to the motion of a pendulum, which he considers as vibrating in a plane; and after showing that the time of oscillation is not sensibly affected, he remarks that upon calculating the force perpendicular to the plane of oscillation, arising from the rotation of the Earth, it is found to be too small sensibly to displace the plane of oscillation or to have any appreciable influence on the motion-a conclusion which, as is well known, is erroneous. He considers also the motion of falling bodies, but the memoir relates principally to the theory of projectiles.

78. That the motion of the spherical pendulum is sensibly affected by the rotation of the Earth is the well-known discovery of Foucault; it appears by his paper, "Démonstration Physique \&c.," Comptes Rendus, t. xxxiI. 1851, that he was led to it by considering the case of a pendulum oscillating at the pole; the plane of oscillation, if actually fixed in space, will by the rotation of the Earth appear to rotate with the same velocity in the contrary direction; and he remarks that although the case of a different latitude is more complicated, yet the result of an apparent rotation of the plane of oscillation, diminishing to zero at the equator, may be obtained either from analytical or from mechanical and geometrical considerations. Some other Notes by Foucault on the subject are given, Comptes Rendus, t. xxxv. (1853).

79. An analytical demonstration of the theorem was given by Binet, Comptes Rendus, t. xxxII. (1851), and by Baehr (1853). Various short papers on the subject will be found in the Philosophical Magazine, and elsewhere.

80. In regard to the above-mentioned problem of falling bodies, we have a Note by W. S., Camb. and Dubl. Math. Journ. t. III. (1848), containing some errors which are rectified in a subsequent paper, "Remarks on the Deviation of Falling bodies, \&c." t. IV. (1849), by Dr Hart and Professor W. Thomson. 
81. The theory of relative motion is considered in a very general manner in M. Quet's memoir, "Des Mouvements relatifs en général \&ic." (1853). Suppose that $x, y, z$ are the coordinates of a particle in relation to a set of moveable axes; let $\xi^{\prime}, \eta^{\prime}, \zeta^{\prime}$ be the coordinates of the moveable origin in reference to a fixed set of axes, and treating the accelerations $\frac{d^{2} \xi^{\prime}}{d t^{2}}, \frac{d^{2} \eta^{\prime}}{d t^{2}}, \frac{d^{2} \zeta^{\prime}}{d t^{2}}$ as if they were coordinates, let these, when resolved along the moveable axes, give $u^{\prime}, v^{\prime}, w^{\prime}$ : suppose, moreover, that $p, q, r$ denote the angular velocities of the system of the moveable axes (or axes of $x, y, z$ ) round the axes of $x, y$, and $z$ respectively; $u^{\prime}, v^{\prime}, w^{\prime}, p, q, r$ are considered as given functions of the time, and then, if

$$
\begin{aligned}
& u=\frac{d^{2} x}{d t^{2}}+2\left(q \frac{d z}{d t}-r \frac{d y}{d t}\right)+z \frac{d q}{d t}-y \frac{d r}{d t}+q(p y-q x)-r(r x-p r)+u^{\prime}, \\
& v=\frac{d^{2} y}{d t^{2}}+2\left(r \frac{d x}{d t}-p \frac{d z}{d t}\right)+x \frac{d r}{d t}-z \frac{d p}{d t}+r(q z-r y)-p(p y-q x)+v^{\prime}, \\
& w=\frac{d^{2} z}{d t^{2}}+2\left(p \frac{d y}{d t}-q \frac{d x}{d t}\right)+y \frac{d p}{d t}-x \frac{d q}{d t}+p(r x-p z)-q(q z-r y)+w^{\prime},
\end{aligned}
$$

it is shown that the equations of motion are to be obtained from the equation

$$
\Sigma m[(u-X) \delta x+(v-Y) \delta y+(w-Z) \delta z]=0,
$$

where $\delta x, \delta y, \delta z$ are the virtual velocities of the particle $m$ in the directions of the moveable axes. This equation is in fact obtained as a transformation of the equation

$$
\Sigma m\left[\left(\frac{d^{2} \xi}{d t^{2}}-X\right) \delta \xi+\left(\frac{d^{2} \eta}{d t^{2}}-Y\right) \delta \eta+\left(\frac{d^{2} \zeta}{d t^{2}}-Z\right) \delta \zeta\right]=0,
$$

which belongs to a set of fixed axes of $\xi, \eta, \zeta$.

82. The equations for the motion of a free particle are of course $u=X, v=Y, w=Z$. In the case where the moveable axes are fixed on the Earth, and moveable with it (the diurnal motion being alone attended to), these lead to equations for the motion of a particle in reference to the Earth, similar to those obtained by Gauss and Poisson. The formulæ are applied to the case of the spherical pendulum, which is developed with some care; and Foucault's theorem of the rotation of the plane of oscillation very readily presents itself. The general formulæ are applied to the relative motion of a solid body, and in particular to the question of the gyroscope; the memoir contains other interesting results.

83. The principal memoirs on the motion of the spherical pendulum, as affected by the rotation of the Earth, are those of Hansen, "Theorie der Pendelbewegung \&c." (1853), which contains an elaborate investigation of all the physical circumstances (resistance of the air, torsion of the string, \&c.) which can affect the actual motion, and the before-mentioned memoir by Dumas, "Ueber der Bewegung des Raumpendels \&c." (1855). The investigation is conducted by means of the variation of the constants; 
81. The theory of relative motion is considered in a very general manner in M. Quet's memoir, "Des Mouvements relatifs en général \&ic." (1853). Suppose that $x, y, z$ are the coordinates of a particle in relation to a set of moveable axes; let $\xi^{\prime}, \eta^{\prime}, \zeta^{\prime}$ be the coordinates of the moveable origin in reference to a fixed set of axes, and treating the accelerations $\frac{d^{2} \xi^{\prime}}{d t^{2}}, \frac{d^{2} \eta^{\prime}}{d t^{2}}, \frac{d^{2} \zeta^{\prime}}{d t^{2}}$ as if they were coordinates, let these, when resolved along the moveable axes, give $u^{\prime}, v^{\prime}, w^{\prime}$ : suppose, moreover, that $p, q, r$ denote the angular velocities of the system of the moveable axes (or axes of $x, y, z$ ) round the axes of $x, y$, and $z$ respectively; $u^{\prime}, v^{\prime}, w^{\prime}, p, q, r$ are considered as given functions of the time, and then, if

$$
\begin{aligned}
& u=\frac{d^{2} x}{d t^{2}}+2\left(q \frac{d z}{d t}-r \frac{d y}{d t}\right)+z \frac{d q}{d t}-y \frac{d r}{d t}+q(p y-q x)-r(r x-p r)+u^{\prime}, \\
& v=\frac{d^{2} y}{d t^{2}}+2\left(r \frac{d x}{d t}-p \frac{d z}{d t}\right)+x \frac{d r}{d t}-z \frac{d p}{d t}+r(q z-r y)-p(p y-q x)+v^{\prime}, \\
& w=\frac{d^{2} z}{d t^{2}}+2\left(p \frac{d y}{d t}-q \frac{d x}{d t}\right)+y \frac{d p}{d t}-x \frac{d q}{d t}+p(r x-p z)-q(q z-r y)+w^{\prime},
\end{aligned}
$$

it is shown that the equations of motion are to be obtained from the equation

$$
\Sigma m[(u-X) \delta x+(v-Y) \delta y+(w-Z) \delta z]=0,
$$

where $\delta x, \delta y, \delta z$ are the virtual velocities of the particle $m$ in the directions of the moveable axes. This equation is in fact obtained as a transformation of the equation

$$
\Sigma m\left[\left(\frac{d^{2} \xi}{d t^{2}}-X\right) \delta \xi+\left(\frac{d^{2} \eta}{d t^{2}}-Y\right) \delta \eta+\left(\frac{d^{2} \zeta}{d t^{2}}-Z\right) \delta \zeta\right]=0,
$$

which belongs to a set of fixed axes of $\xi, \eta, \zeta$.

82. The equations for the motion of a free particle are of course $u=X, v=Y, w=Z$. In the case where the moveable axes are fixed on the Earth, and moveable with it (the diurnal motion being alone attended to), these lead to equations for the motion of a particle in reference to the Earth, similar to those obtained by Gauss and Poisson. The formulæ are applied to the case of the spherical pendulum, which is developed with some care; and Foucault's theorem of the rotation of the plane of oscillation very readily presents itself. The general formulæ are applied to the relative motion of a solid body, and in particular to the question of the gyroscope; the memoir contains other interesting results.

83. The principal memoirs on the motion of the spherical pendulum, as affected by the rotation of the Earth, are those of Hansen, "Theorie der Pendelbewegung \&c." (1853), which contains an elaborate investigation of all the physical circumstances (resistance of the air, torsion of the string, \&c.) which can affect the actual motion, and the before-mentioned memoir by Dumas, "Ueber der Bewegung des Raumpendels \&c." (1855). The investigation is conducted by means of the variation of the constants; 
the integrals for the undisturbed problem were, as already noticed, obtained by means of Jacobi's Principal Function, that is, in a form which leads at once to the expressions for the variation of the constants; and the investigation appears to be carried out in a most elaborate and complete manner.

84. In concluding this part of the subject I refer to Mr Worm's work, The Rotation of the Earth (1862), where the last-mentioned questions (falling bodies, the pendulum, and the gyroscope) are, in reference to the proofs they afford of the rotation of the Earth, considered as well in an experimental as in a mathematical point of view. The second part of the volume contains the theory (after Laplace and Gauss) of falling bodies, that of the pendulum (after Hansen), and that of the gyroscope (after Yvon Villarceau); and the whole appears to be a complete and satisfactory résumé of the experimental and mathematical theories to which it relates.

85. We have also Cohen "On the Differential Coefficients and Determinants of Lines \&c." (1862), where the equations for relative motion are obtained in a very elegant manner. The fundamental notion of the memoir may be considered to be the dealing directly with lines, velocities, \&c., which are variable in direction as well as in magnitude, instead of referring them, as in the ordinary analytical method, to axes fixed in space. The memoir is a highly interesting and valuable one, and the results are brought out with great facility; but I cannot but think that the great care required to apply the method correctly is an objection to it, if used otherwise than by way of interpretation of previously obtained results, and that the ordinary method is preferable.

I may remark that the theory of relative motion connects itself with the lunar and planetary theories as regards the reference of the plane of the orbit to the variable ecliptic, and as regards the variations of the position of the orbit; but this is a subject which I have abstained from entering upon.

Miscellaneous Problems. Articles Nos. 86 to 111 (several subheadings).

\section{Motion of a single particle.}

86. Jacobi, in the memoir "De Motu puncti singularis" (1842), notices ( $§ 5$ ) the case of a body acted on by a central force which is any homogeneous function of the degree -2 of the coordinates; or representing these by $r \cos \phi, r \sin \phi$, then the force is $=\frac{\Phi}{r^{2}}$, where $\Phi$ is any function of the angle $\phi$. In fact, after integrating by a process different from the ordinary one the case of a central force $\propto \frac{1}{r^{2}}$, he remarks that the method in fact applies to the more general law of force just mentioned.

87. Jacobi, in the memoir "Theoria Novi Multiplicatoris \&c." (1845), considers (§ 25) the case of a body acted on by a central force $P$ a function of the distance, and C. IV. 
besides by forces $X$ and $Y$, which are homogeneous functions of the degree -3 of the coordinates $(x, y)$; viz. the equations of motion are in this case

$$
\begin{aligned}
& \frac{d^{2} x}{d t^{2}}=-\frac{P x}{r}+X, \\
& \frac{d^{2} y}{d t^{2}}=-\frac{P y}{r}+Y,
\end{aligned}
$$

and there is an integral

$$
\frac{1}{2}\left(x y^{\prime}-x^{\prime} y\right)^{2}-\int x^{2}(x Y-y X) d \frac{y}{x}=\text { const. }
$$

(the function under the integral sign is obviously a function of the degree 0 in $(x, y)$, that is, it is a function of $\frac{y}{x}$ ). If $X, Y$ are the derived functions of a force-function $U$ of the degree -2 in $(x, y)$, then there is, besides, the integral of Vis Viva, and thence a third integral is obtained by means of the theorem of the Ultimate Multiplier. It may be noticed that in the last-mentioned case the force-function is of the form $\frac{\Phi}{r^{2}}$, so that if we represent also the central force by means of a force-function $R$ (= function of $r$ ), then the entire force-function is $R+\frac{\Phi}{r^{2}}$. The case is a very interesting one; it includes that considered § iv. of Bertrand's "Mémoire sur les équations différentielles de la Mécanique" (1852), where the force-function is of the form $=\frac{\Phi}{r^{2}}$.

\section{Motion of three mutually attracting bodies in a right line.}

88. The problem is considered by Euler in the memoir "De Motu rectilineo \&c." (1765), the forces being as the inverse square of the distance; and a solution is obtained for an interesting particular case. Let $A, B, C$ be the masses, and suppose that at the commencement of the motion the distances $C B, B A$ are in the ratio $\alpha: 1$, and that the velocities (assumed to be in the same sense) are proportional to the distances from a fixed point. Then, if $\alpha$ be the real root (there is only one) of the equation of the fifth order

$$
C\left(1+3 \alpha+3 \alpha^{2}\right)=A \alpha^{3}\left(\alpha^{2}+3 \alpha+3\right)+B\left(\alpha+1^{2}\right)\left(\alpha^{3}-1\right),
$$

the distances $C B, B A$ will always continue in the ratio $\alpha: 1$. It may be added that the distances $C B, B A$ each of them vary as $r^{2}-a^{2}$, where $a$ is a constant, and $r$ is, according to the initial circumstances, a function of $t$ defined by one or the other of the two equations

$$
\begin{aligned}
& t=n^{3} r \sqrt{r^{2}-a^{2}}-n^{3} a^{2} \log \frac{r+\sqrt{r^{2}-a^{2}}}{a}, \\
& t=n^{3} r \sqrt{a^{2}-r^{2}}+n^{3} a^{2} \sin ^{-1} \frac{r}{a} .
\end{aligned}
$$


89. The bodies are considered as restricted to move in a given line; but it is clear that if the bodies, considered as free points in space, are initially in a line, and the initial velocities are also in this line, then the bodies will always continue in this line, which will be a fixed line in space. But if the distances and velocities are as above, except only that the velocities, instead of being along the line, are parallel to each other in any direction whatever, then the bodies will always continue in a line, which is in this case a moveable line in space (see post, No. 93).

90. Euler resumes the problem in the memoir of 1776 in the Nova Acta Petrop. The distances $A B, B C$ being $p$ and $q$, then

$$
\begin{aligned}
& \frac{d^{2} p}{d t^{2}}=-\frac{A+B}{p^{2}}+\frac{C}{q^{2}}-\frac{C}{(p+q)^{2}}, \\
& \frac{d^{2} q}{d t^{2}}=\frac{A}{p^{2}}-\frac{A}{(p+q)^{2}}-\frac{B+C}{q^{2}}
\end{aligned}
$$

and in particular he considers the before-mentioned case of a solution of the form $p=n q$; and also the particular problem where one of the masses vanishes, $C=0$; in this case, introducing (instead of $p, q$ ), the new variables $u, s$, where $q=u p, d q=s d p$ (a transformation suggested by the homogeneity of the equations), and making, moreover, the particular supposition that the integral of the first equation is $\left(\frac{d p}{d t}\right)^{2}=\frac{2(A+B)}{p}$ (viz. making the constant of integration to vanish), he obtains between $s$ and $u$ the equation of the first order

$$
2(A+B) \frac{d s}{d u}(s-u)=(A+B) s+A-\frac{A}{(1+u)^{2}}-\frac{B}{u^{2}},
$$

which, however, he is not able to integrate.

91. Jacobi has given in the memoir "Theoria, Novi Multiplicatoris \&c." (1845) (§ 28, entitled "De Problemate trium corporum in eâdem rectâ motorum. Substitutio Euleriana. Theoremata de viribus homogeneis") a very symmetrical and elegant investigation of the same problem. The centre of gravity being assumed to be at rest, the coordinates $x, x_{1}, x_{2}$ of the three bodies are in the first instance expressed as linear functions of the two variables $u, v$ (being, as Jacobi remarks, the transformation employed in his memoir "Sur l'élimination des Nœuds" (1843), post, No. 114), $\frac{d^{2} u}{d t^{2}}$ and $\frac{d^{2} v}{d t^{2}}$ come out respectively equal to homogeneous functions of the degree -2 of these variables $u$ and $v$, and the integral of Vis Viva exists. The subsequent transformation consists in the introduction of the variables $r, \phi, s, \eta$, where $u=r \cos \phi, v=r \sin \phi, s=\sqrt{r} \frac{d r}{d t}, \eta=\sqrt{r^{3}} \frac{d \phi}{d t}$; this gives a system of equations independent of $r$; viz.,

$$
d \phi: d s: d \eta=\eta: \frac{1}{2} s^{2}+\eta^{2}-\Phi:-\frac{1}{2} s \eta+\Phi^{\prime},
$$

where $\Phi$ is a given function of $\phi$, and $\Phi^{\prime}$ is the derived function. If these equations were integrated, the equation of Vis Viva gives at once $r=\frac{1}{h}\left(\Phi-\frac{1}{2}\left(s^{2}+\eta^{2}\right)\right)$; and 
finally the time $t$ would be given by a quadrature. The system of three equations has the multiplier $M=\frac{1}{\sqrt{\phi-\frac{1}{2}\left(s^{2}+\eta^{2}\right)}}$, hence if one integral were known the other would be at once furnished by the general theory. There is a simplification in the form of the solution if $h$ (the constant of Vis Viva) $=0$. It is remarked that the method is equally applicable when the force varies as any power of the distance; and moreover that when the force varies as $(\text { dist. })^{-3}$, then the solution depends upon one quadrature only.

92. The concluding part of the section relates to the very general problem of a system of $n$ particles acted on by any forces homogeneous functions of the coordinates (this includes the case of $n$ particles mutually attracting each other according to a power of the distance), and this more general investigation illustrates the method employed in regard to the three bodies in a line. It may be remarked that in the general theorem for the $n$ particles "sint vires \&c.," the constant of Vis Viva is supposed to vanish.

\section{Particular cases of the motion of three bodies.}

93. In the case of three bodies attracting each other according to the inverse square of the distance, the bodies may move in such manner as to be constantly in a line (a moveable line in space); this appears by the memoir, Euler, "Considérations générales \&c." (1764), in which memoir, however (which it will be observed precedes the memoir "De Motu rectilineo \&c." (1765), referred to No. 88), Euler assumes that the mass of one of the bodies is so small as not to affect the relative motion of the other two. Calling the bodies the Sun, Earth, and Moon, and taking the masses to be $1, m, 0$, then a result obtained is, that in order that the Moon may be perpetually in conjunction, its distance must be to that of the Sun as $\alpha: 1$, where $m(1-\alpha)^{2}=3 \alpha^{3}-3 \alpha^{4}+\alpha^{5}$, or $\alpha=\sqrt[3]{\frac{1}{3} m}$ nearly. It appears, however (antè, No. 88), that the foregoing restriction as to the masses is unnecessary, and, as will be mentioned, the problem has since been treated without such restriction. Euler investigates the motion in the case where the initial circumstances are nearly but not exactly as originally supposed; this assumes, however, that the motion is stable-i.e. that the bodies will continue to move nearly, but not exactly as originally supposed, which is at variance with the conclusions of Liouville's memoir, post, No. 95. I have not examined the cause of this discrepancy.

94. In the Mécanique Céleste (1799), Book X. c. 6, Laplace considers two cases where the motion can be exactly determined.

$1^{\circ}$. Force varies as any function of the distance. It is shown that the motion may be such that the bodies form always an equilateral triangle of variable magnitudethe motion of each body about the centre of gravity being the same as if that point were a centre of force attracting the body according to a similar law.

$2^{\circ}$. Force $\propto(\text { dist. })^{\mathrm{n}}$. The motion may be such that the three bodies are always in a right line (moveable in space), the relative distances being in fixed ratios to each 
other. In particular, if force $\propto(\text { dist. })^{-2}$, then $m, m^{\prime}, m^{\prime \prime}$ being the masses, the quantity $z$ which determines the ratio of the distances $m^{\prime \prime} m^{\prime}, m^{\prime} m$ is given by

$$
0=m z^{2}\left[(1+z)^{3}-1\right]-m^{\prime}(1+z)^{2}\left(1-z^{3}\right)-m^{\prime \prime}\left[(1+z)^{3}-z^{3}\right]=0,
$$

which is, in fact, the formula in Euler's memoir "De Motu rectilineo \&c."

95. Liouville's memoir "Sur un cas particulier \&c." (1842) has for its object to show that if the initial circumstances are not precisely as supposed in the second of the two cases considered by Laplace, or, what is the same thing, in Euler's memoir "Considérations générales \&c.," then the motion is unstable; the instability manifests itself in the usual manner, viz. the expressions for the deviations from the normal positions are found to contain real exponentials which increase indefinitely with the time.

96. It may be proper to refer here to Jacobi's theorem, Comptes Rendus, t. III. p. 61 (1836), quoted in the foot-note to No. 31 of my Report of 1857, [195], which relates to the motion of a point without mass revolving round the Sun, and disturbed by a planet moving in a circular orbit, and properly belongs (as I have there remarked) to the problem of two centres, one of them moveable and the other revolving round it in a circle with uniform velocity. The theorem (given without demonstration by Jacobi) is proved by Liouville in his last-mentioned memoir, and he remarks that the theorem follows very simply as a corollary of the theorem by Coriolis, "Mémoire sur le principe des forces vives dans les mouvemens relatifs des Machines," Journ. de l'Ecole Polyt. t. xIII. pp. 268-302 (1832). There is, however, no difficulty in proving the theorem; another proof is given, Cayley, "Note on a Theorem of Jacobi's \&c." (1862).

\section{Motion in a resisting medium.}

97. I do not consider the various integrable cases of the motion of a particle in a resisting medium, the resistance varying with the velocity according to some assumed law, the particle being either not acted on by any force, or acted upon by gravity only. Some interesting cases are considered in Jacobi's memoir "De Motu puncti singularis" (1842), $\S 6$ and 7 (see post, No. 108).

98. In the case of a central force varying as (dist. $)^{-2}$, the effect of a resisting medium $\left(R \propto v^{2}\right)$ is considered in reference to the lunar theory, in the Mécanique Céleste, Book VII. c. 6. Formulæ for the variations of the elliptic elements are given in the Mécanique Analytique, t. II. (2nd edition). But the variations of the elliptic elements are fully worked out by means of elliptic and Jacobian functions in Sohncke's valuable memoir "Motus Corporum \&c." (1833).

99. The effect of the resistance of the air on a pendulum has been elaborately considered by Poisson, Bessel, Stokes, and others; as the dimensions of the ball are attended to, the problem is in fact a hydrodynamical one.

The effect on the spherical pendulum is considered in Hansen's memoir "Theorie der Pendelbewegung \&c. (1853).

The effect on the motion of a projectile is considered in Poisson's memoirs "Sur le Mouvement des Projectiles \&c." (1838). 
Liouville's memoirs "Sur quelques Cas particuliers où les équations du mouvement d'un point matériel peuvent s'intégrer" (1846-49).

100. In the first memoir ( $(1)$ the author considers a point moving in a plane or on a given surface, where the principle of Vis Viva holds good (or say where there is a force-function $U$ ). The coordinates of the point, and the function $U$, may be expressed in terms of two variables $\alpha, \beta$, and it is assumed that these are such that

$$
d s^{2}=\lambda\left(d \alpha^{2}+d \beta^{2}\right)
$$

where $\lambda$ is a function of $\alpha$ and $\beta$. That is, we have $T=\frac{1}{2} \lambda\left(\alpha^{\prime 2}+\beta^{\prime 2}\right)$; and the equations of motion are

$$
\begin{aligned}
& \frac{d \cdot \lambda \alpha^{\prime}}{d t}=\frac{1}{2} \frac{d \lambda}{d \alpha}\left(\alpha^{\prime 2}+\beta^{\prime 2}\right)+\frac{d U}{d \alpha}, \\
& \frac{d \cdot \lambda \beta^{\prime}}{d t}=\frac{1}{2} \frac{d \lambda}{d \beta}\left(\alpha^{\prime 2}+\beta^{\prime 2}\right)+\frac{d U}{d \beta} .
\end{aligned}
$$

One integral of these is

$$
\lambda\left(\alpha^{\prime 2}+\beta^{\prime 2}\right)=2 U+C
$$

and by means of it the equations take the form

$$
\begin{aligned}
& \frac{d \cdot \lambda \alpha^{\prime}}{d t}=\frac{1}{2 \lambda} \frac{d \lambda}{d \alpha}(2 U+C)+\frac{d U}{d \alpha}, \\
& \frac{d \cdot \lambda \beta^{\prime}}{d t}=\frac{1}{2 \lambda} \frac{d \lambda}{d \beta}(2 U+C)+\frac{d U}{d \beta} .
\end{aligned}
$$

These equations, it is easy to show, may be integrated if

$$
(2 U+C) \lambda=f \alpha-F \beta,
$$

and they then in fact give

$$
\begin{aligned}
& \lambda^{2} \alpha^{\prime 2}=f \alpha-A, \\
& \lambda^{2} \beta^{\prime 2}=A-F \beta,
\end{aligned}
$$

where $A$ is an arbitrary constant. And we then have

$$
\frac{d \alpha}{\sqrt{f \alpha-A}}=\frac{d \beta}{\sqrt{A-F \beta}}
$$

which gives the path, and the expression for the time is easily obtained by means of a quadrature.

It is not more general, but it is frequently convenient to employ instead of $\alpha, \beta$, two variables $\mu$ and $\nu$, such that

$$
d s^{2}=\lambda\left(m d \mu^{2}+n d \nu^{2}\right)
$$


where $m$ is a function of $\mu$ only and $n$ of $\nu$ only, while $\lambda$ contains $\mu$ and $\nu$. The geometrical signification of the equation $d s^{2}=\lambda\left(d \alpha^{2}+d \beta^{2}\right)$, or of the last-mentioned equivalent form, is that the curves

intersect at right angles.

$$
\alpha \text { or } \lambda=\text { const., } \quad \beta \text { or } \mu=\text { const. }
$$

The foregoing differential equation of the path, writing $f \mu, F \nu$ in the place of $f \alpha, F \beta$ respectively, may be expressed in the form

$$
f \mu \cos ^{2} i+F \nu \sin ^{2} i=A,
$$

where $i, 90^{\circ}-i$ are the inclinations of the path at the point $(\lambda, \mu)$ to the two orthotomic curves through this point.

101. The before-mentioned equation

$$
(2 U+C) \lambda=f \alpha-F \beta
$$

may be satisfied independently of $C$, or else only for a particular value of $C$. In the former case the law of force is much more restricted, but on the other hand there is no restriction as regards the initial circumstances of the motion; it is the more important one, and is alone attended to in the sequel of the memoir. In the case in question (changing the functional symbols) we must have

$$
\lambda=\phi \alpha-\sigma \beta, \quad \lambda U=f \alpha-F \beta ;
$$

so that the functions denoted above by $f \alpha, F \beta$ now are $2 f \alpha+C \phi \alpha, 2 F \beta+C \varpi \beta$; the equation of the trajectory is

$$
\frac{d \alpha}{\sqrt{2 f \alpha+C \phi \alpha-A}}=\frac{d \beta}{\sqrt{A-2 F \beta+C \varpi \beta}},
$$

and for the time the formula is

$$
d t=\frac{\phi \alpha d \alpha}{\sqrt{2 f \alpha+C \phi \alpha-A}}-\frac{\varpi \beta d \beta}{\sqrt{A-2 F \beta+C \omega \beta}} .
$$

It is noticed also that taking $B, \epsilon$ to denote two new arbitrary constants, and writing

$$
\Theta \int d \alpha \sqrt{2 f \alpha+C \phi \alpha-A}+\int d \beta \sqrt{A-2 F \beta+C \varpi \beta},
$$

the equation of the trajectory and the expression for the time assume the forms

$$
\frac{d \Theta}{d A}=B, \quad t=2 \frac{d \Theta}{d C}+\epsilon,
$$

as is known à priori by a theorem of Jacobi's.

If the forces vanish, the path is a geodesic line; and denoting by $a$ the ratio of the constants $A, C$, we have

and moreover

$$
\frac{d \alpha}{\sqrt{\phi \alpha-a}}=\frac{d \beta}{\sqrt{a-\sigma \beta}}
$$

$$
d s=d \alpha \sqrt{\phi \alpha-a}+d \beta \sqrt{a-\phi \beta},
$$

which are geometrical properties relating to the geodesic line. 
102. Passing to the applications: in the first place, if $\alpha, \beta$ are rectangular coordinates of a point in plano, then writing instead of them $x, y$, we have $d s^{2}=d x^{2}+d y^{2}$, which is of the required form; but the result obtained is the self-evident one, that the equations may be integrated by quadratures when $U$ is of the form funct. $x$-funct. $y$.

But taking instead the elliptic coordinates $\mu, \nu$, of a point in plano-viz., as employed by the author, these are the semiaxes of the confocal ellipse and hyperbola represented by the equations

$$
\frac{x^{2}}{\mu^{2}}+\frac{y^{2}}{\mu^{2}-b^{2}}=1, \quad \frac{x^{2}}{\nu^{2}}-\frac{y^{2}}{b^{2}-\nu^{2}}=1,
$$

- very interesting results are obtained. The equations give

and thence

$$
b^{2} x^{2}=\mu^{2} \nu^{2}, \quad b^{2} y^{2}=\left(\mu^{2}-b^{2}\right)\left(b^{2}-\nu^{2}\right),
$$

$$
d s^{2}=\left(\mu^{2}-\nu^{2}\right)\left(\frac{d \mu^{2}}{\mu^{2}-b^{2}}+\frac{d \nu^{2}}{\nu^{2}-b^{2}}\right),
$$

which is of the proper form, and the corresponding expression of $U$ is

$$
U=\frac{f \mu-F \nu}{\mu^{2}-\nu^{2}}
$$

so that the force-function having this value $(f \mu, F \nu$ being arbitrary-functions of $\mu$ and $\nu$ respectively), the equations of motion may be integrated by quadratures.

103. In particular, if

$$
\begin{aligned}
& f \mu=g \mu+g^{\prime} \mu+k\left(\mu^{4}-b^{2} \mu^{2}\right), \\
& F^{\prime} \nu=g \nu-g^{\prime} \nu+k\left(\nu^{4}-b^{2} \nu^{2}\right),
\end{aligned}
$$

then

$$
U=\frac{g}{\mu+\nu}+\frac{g^{\prime}}{\mu-\nu}+k\left(\mu^{2}+\nu^{2}-b^{2}\right)
$$

But $\mu+\nu, \mu-\nu$ are the distances of the point from the two foci, and $\mu^{2}+\nu^{2}-b^{2}\left(=x^{2}+y^{2}\right)$. is the square of the distance from the centre, so that the expression for $U$ is

$$
U=\frac{g}{r}+\frac{g^{\prime}}{r^{\prime}}+k R^{2}
$$

and the case is that of forces to the foci varying inversely as the squares of the distances, and a force to the centre varying directly as the distance-the case considered by Lagrange in the problem of two centres. But this is merely one particular case of those given by the general formula.

The cases $g=0, g^{\prime}=0, k=0$ (no forces), and $g=0, g^{\prime}=0$ (a force to the centre) lead to some interesting results; it is noticed also that the expression for the forcefunction may be written $U=\frac{\text { funct. }\left(r+r^{\prime}\right)-\text { funct. }\left(r-r^{\prime}\right)}{r r^{\prime}}$, and that it may be thereby ascertained (without transforming to elliptic coordinates) whether a given value of the force-function is of the form considered in the theory. 
In $\S 3$ the author considers the expression $d x^{2}+d y^{2}=\lambda\left(d \alpha^{2}+d \beta^{2}\right)$, $\lambda$ being in the first instance any function whatever of $\alpha$ and $\beta$; and he shows that the expressions of $x, y$ are given by the equation

$$
x+y \sqrt{-1}=\psi(\alpha+\beta \sqrt{-1}),
$$

$\psi$ being any real function. If, however, it is besides assumed that $\lambda$ is of the required form $=f \alpha-F \beta$, then he shows that the system of elliptic coordinates is the only one for which the conditions are satisfied. $\$ \S 4,5,6$ and 7 relate to the motion of a point on a sphere, an ellipsoid, a surface of revolution, and the skew helicoid respectively; and the concluding $\S 8$ contains only a brief reference to the author's second memoir.

104. Liouville's second and third memoirs may be more briefly noticed. In the second memoir the author starts from Jacobi's theorem of the $V$ function, viz., assuming that there is a force-function $U$ independent of the time, then in order to integrate the equations of motion $\left(\frac{d^{2} x}{d t^{2}}=\frac{d U}{d x}, \frac{d^{2} y}{d t^{2}}=\frac{d U}{d y}, \frac{d^{2} z}{d t^{2}}=\frac{d U}{d z}\right)$, all that is required is to find a function $\Theta$ of $x, y, z$ containing three arbitrary constants $A, B, C$ (distinct from the constant attached to $\Theta$ by mere addition) satisfying the differential equation

$$
\left(\frac{d \Theta}{d x}\right)^{2}+\left(\frac{d \Theta}{d y}\right)^{2}+\left(\frac{d \Theta}{d z}\right)^{2}=2(U+C)
$$

for then the required integrals of the equations of motion are

$$
\frac{d \Theta}{d A}=A^{\prime}, \quad \frac{d \Theta}{d B}=B^{\prime}, \quad \frac{d \Theta}{d C}=t+C^{\prime},
$$

$A^{\prime}, B^{\prime}, C^{\prime}$ being new arbitrary constants. Liouville introduces in place of $x, y, z$, the elliptic coordinates $\rho, \mu, \nu$, which are such that

$$
\begin{aligned}
& \frac{x^{2}}{\rho^{2}}+\frac{y^{2}}{\rho^{2}-b^{2}}+\frac{z^{2}}{\rho^{2}-c^{2}}=1, \\
& \frac{x^{2}}{\mu^{2}}+\frac{y^{2}}{\mu^{2}-b^{2}}-\frac{z^{2}}{c^{2}-\mu^{2}}=1, \\
& \frac{x^{2}}{\nu^{2}}-\frac{y^{2}}{b^{2}-\nu^{2}}-\frac{z^{2}}{c^{2}-\nu^{2}}=1,
\end{aligned}
$$

or, what is the same thing,

$$
\begin{aligned}
& x=\frac{\rho \mu \nu}{b c}, \\
& y=\frac{\sqrt{\rho^{2}-b^{2}} \sqrt{\mu^{2}-b^{2}} \sqrt{b^{2}-\nu^{2}}}{b \sqrt{c^{2}-b^{2}}} \\
& z=\frac{\sqrt{\rho^{2}-c^{2}} \sqrt{c^{2}-\mu^{2}} \sqrt{c^{2}-\nu^{2}}}{c \sqrt{c^{2}-b^{2}}}
\end{aligned}
$$

C. IV. 
and he then finds that the resulting partial differential equation in $\rho, \mu, \nu$ may be integrated provided that $U$ is of the form

$$
U=\frac{\left(\mu^{2}-\nu^{2}\right) f \rho+\left(\rho^{2}-\nu^{2}\right) F \mu+\left(\rho^{2}-\nu^{2}\right) \varpi \nu}{\left(\rho^{2}-\mu^{2}\right)\left(\rho^{2}-\nu^{2}\right)\left(\mu^{2}-\nu^{2}\right)}
$$

$f, F$, w being any functional symbols whatever ; viz., the expression for $\Theta$ is

$$
\begin{aligned}
\Theta= & \int d \rho \sqrt{\frac{2 f \rho+A+B \rho^{2}+2 C \rho^{4}}{\left(\rho^{2}-b^{2}\right)\left(\rho^{2}-c^{2}\right)}} \\
& +\int d \mu \sqrt{\frac{2 F \mu+A+B \mu^{2}+2 C \mu^{4}}{\left(\mu^{2}-b^{2}\right)\left(c^{2}-\mu\right)^{2}}} \\
& +\int d \nu \sqrt{\frac{2 \varpi \nu+A+B \nu^{2}+2 C \nu^{4}}{\left(b^{2}-\nu^{2}\right)\left(c^{2}-\nu^{2}\right)}}
\end{aligned}
$$

In the case where $U=0$ we have a particle not acted on by any forces, and the path is of course a straight line. The peculiar form in which these equations are obtained leads to very interesting results in regard to the theory of Abelian integrals, and to that of the geodesic lines of an ellipsoid.

The formulæ require to be modified in certain cases, such as $c=b$ or $b=0$. The case $b=0$ leads to the theory developed in the first memoir in relation to the problem of two centres. The case is indicated where $b=0, c=0$, the ratio $b: c$ remaining finite.

The case is briefly considered of a particle moving on a given surface.

105. The third memoir purports to relate to a system of particles, but the formulæ are exhibited under a purely analytical point of view; so much so, that the coordinates of the points ( 3 for each point) are considered as forming a single system of variables $x_{1}, x_{2}, \ldots x_{i}$. The partial differential equation is

$$
\left(\frac{d \Theta}{d x_{1}}\right)^{2}+\left(\frac{d \Theta}{d x_{2}}\right)^{2} \ldots+\left(\frac{d \Theta}{d x_{i}}\right)^{2}=2(U+h),
$$

which is transformed by introducing therein the new variables $\rho_{1}, \rho_{2}, \ldots \rho_{i}$ analogous to the elliptic coordinates of the second memoir. The memoir really belongs rather to the theory of the Abelian integrals (in regard to which it appears to be a very valuable one) than to dynamics.

Memoirs by Jacobi, Bertrand, and Donkin, relating to various Special Problems.

106. I have inserted this heading for the sake of showing at a single view what are the special problems incidentally considered in the undermentioned memoirs which are referred to in several places in the present Report. 
and he then finds that the resulting partial differential equation in $\rho, \mu, \nu$ may be integrated provided that $U$ is of the form

$$
U=\frac{\left(\mu^{2}-\nu^{2}\right) f \rho+\left(\rho^{2}-\nu^{2}\right) F \mu+\left(\rho^{2}-\nu^{2}\right) \varpi \nu}{\left(\rho^{2}-\mu^{2}\right)\left(\rho^{2}-\nu^{2}\right)\left(\mu^{2}-\nu^{2}\right)}
$$

$f, F$, w being any functional symbols whatever: viz., the expression for $\Theta$ is

$$
\begin{aligned}
\Theta= & \int d \rho \sqrt{\frac{2 f \rho+A+B \rho^{2}+2 C \rho^{4}}{\left(\rho^{2}-b^{2}\right)\left(\rho^{2}-c^{2}\right)}}, \\
& +\int d \mu \sqrt{\frac{2 F \mu+A+B \mu^{2}+2 C \mu^{4}}{\left(\mu^{2}-b^{2}\right)\left(c^{2}-\mu\right)^{2}}} \\
& +\int d \nu \sqrt{\frac{2 \varpi \nu+A+B \nu^{2}+2 C \nu^{4}}{\left(b^{2}-\nu^{2}\right)\left(c^{2}-\nu^{2}\right)}} .
\end{aligned}
$$

In the case where $U=0$ we have a particle not acted on by any forces, and the path is of course a straight line. The peculiar form in which these equations are obtained leads to very interesting results in regard to the theory of Abelian integrals, and to that of the geodesic lines of an ellipsoid.

The formulæ require to be modified in certain cases, such as $c=b$ or $b=0$. The case $b=0$ leads to the theory developed in the first memoir in relation to the problem of two centres. The case is indicated where $b=0, c=0$, the ratio $b: c$ remaining finite.

The case is briefly considered of a particle moving on a given surface.

105. The third memoir purports to relate to a system of particles, but the formulæ are exhibited under a purely analytical point of view; so much so, that the coordinates of the points ( 3 for each point) are considered as forming a single system of variables $x_{1}, x_{2}, \ldots x_{i}$. The partial differential equation is

$$
\left(\frac{d \Theta}{d x_{1}}\right)^{2}+\left(\frac{d \Theta}{d x_{2}}\right)^{2} \ldots+\left(\frac{d \Theta}{d x_{i}}\right)^{2}=2(U+h)
$$

which is transformed by introducing therein the new variables $\rho_{1}, \rho_{2}, \ldots \rho_{i}$ analogous to the elliptic coordinates of the second memoir. The memoir really belongs rather to the theory of the Abelian integrals (in regard to which it appears to be a very valuable one) than to dynamics.

Memoirs by Jacobi, Bertrand, and Donkin, relating to various Special Problems.

106. I have inserted this heading for the sake of showing at a single view what are the special problems incidentally considered in the undermentioned memoirs which are referred to in several places in the present Report. 
107. Jacobi, "De Motu puncti singularis" (1842).-I call to mind that the memoir chiefly depends on the theorem of the Ultimate Multiplier (the theory in its generality being developed in the later memoir "Theoria Novi Multiplicatoris \&c.," 1844-45). $\S 4$ is entitled "The motion of a point on the surface of revolution," which, the principle of the conservation of areas holding good, is reduced to the problem of the motion on the meridian curve, and thus depends upon quadratures only. $\S 5$ is entitled "On the motion of a point about a fixed centre attracted according to a certain law more general than the Newtonian one" (antè, No. 85). §6, "On the motion of a point on a given curve and in a resisting medium" (resistance $=a+b e^{c v^{2}}$, or $=a+b v^{2}$ ); and $\S 7$, "On the Ballistic Curve," viz., the forces are gravity and a resistance $=a+b v^{n}$.

108. In Jacobi's memoir "Theoria Novi Multiplicatoris \&c." (1845), § 25 is entitled "On the motion of a point attracted towards a fixed centre" (see antè, No 87); $\S 26$, "On the motion of a point attracted towards two fixed centres according to the Newtonian law" (antè, No. 56); $§ 27$, "On the rotation of a solid body about a fixed point" (post, No. 193); $§ 28$, "On the problem of three bodies moving in a right line; the Eulerian substitution; theorems on homogeneous forces" (antè, No. 91); and § 29, "The principle of the ultimate multiplier applied to a free system of material points moving in a resisting medium; on the motion of a comet in a resisting medium about the sun."

109. And in Jacobi's memoir "Nova Methodus \&c." (1862), besides $\S 64$ and $\S 65$, which are applications of the method to general dynamical theorems, we have $\S 66$, containing a simultaneous solution of the problem of the motion of a point attracted to a fixed centre and of that of the rotation of a solid body (post, No. 206) and $\S 67$, relating to the motion of a point attracted to a fixed centre according to the Newtonian law.

110. Bertrand's "Mémoire sur les intégrales différentielles de la Mécanique " (1852).$\S$ III. relates to the motion of a point attracted to a fixed centre by a force varying as a function of the distance; $\S$ IV. to the case where the forces arise from a forcefunction $U=\frac{1}{x^{2}+y^{2}} \phi\left(\frac{x}{y}\right)$ (or, what is the same thing, $\left.=\frac{\Phi}{r^{2}}\right)$ (antè, No. 87 ); $\S$ V. to the problem of two centres (antè, No. 62), and $\S$ VI. to the problem of three bodies (post, No. 117).

111. Donkin's memoir "On a Class of Differential Equations \&c." (1855). Part I. Nos. 27 to 30 relate to the problem of central forces (in space), No. 31 to the rotation of a solid body, and $\S$ III. to the same subject, viz. Nos. 40 and 41 to the general case, Nos. 42 to 44 to the particular case $A=B$; and Nos. 45 to 48 to the reduction thereto of the general case by treating the forces which arise from the inequality of $A$ and $B$ as disturbing forces. Part II. Nos. 59 and 60 relate to the spherical pendulum; Nos. 72 and 73 to "Transformation from fixed to moving axes of coordinates," say to Relative Motion; and Nos. 84 to 96 to the problem of three bodies (post, No. 120). 
The Problem of Three Bodies, Article Nos. 112 to 123.

112. A system of differential equations, such as

$$
\frac{d x_{1}}{X_{1}}=\frac{d x_{2}}{X_{2}} \ldots=\frac{d x_{n+1}}{X_{n+1}}
$$

( $n$ equations between $n+1$ variables), may be termed a system of the $n$th order, or more simply a system of $n$ equations. Let $\left(u_{1}, u_{2} \ldots u_{n+1}\right)$ be any functions of the original variables $\left(x_{1}, x_{2} \ldots . x_{n+1}\right)$, the system may be transformed into the similar system

$$
\frac{d u_{1}}{U_{1}}=\frac{d u_{2}}{U_{2}} \cdots=\frac{d u_{n+1}}{U_{n+1}}
$$

and if it happens that we have e.g. $U_{1}$ identically equal to zero, then the system becomes

$$
d u_{1}=0, \quad\left(\frac{d u_{2}}{U_{2}}=\frac{d u_{3}}{U_{3}} \ldots=\frac{d u_{n+1}}{U_{n+1}}\right)
$$

so that we have an integral $u_{1}=c$, and then in the remaining equations substituting this value, or treating $u_{1}$ as constant, the system is reduced to one of $m-1$ equations. Or again, if it happen that we have in the transformed system $m$ equations $(m<n)$, say

$$
\frac{d u_{1}}{U_{1}}=\frac{d u_{2}}{U_{2}} \ldots=\frac{d u_{m+1}}{U_{m+1}}
$$

which are such that $U_{1}, U_{2} \ldots U_{m+1}$ are functions of only the $m+1$ variables $u_{1}, u_{2} \ldots u_{m+1}$, then the integration of the proposed system of $n$ equations depends on the integration in the first instance of a system of $m$ equations. It is to be observed that if the system of $m$ equations can be integrated, then the completion of the integration of the original system depends on the integration of a system of $n-m$ equations, and in this sense the original system of $n$ equations may be said to be broken up into two systems of $m$ equations and $n-m$ equations respectively: but non constat that the system of $m$ equations admits of integration; and it is therefore more correct to say that, from the original system of the $n$ equations, there has been separated off a system of $m$ equations.

113. The bearing of the foregoing remarks on the problem of three bodies will presently appear. It will be seen that whereas the problem as it stood before Jacobi depends on a system of seven equations, it has been shown by him that there may be separated off from this a system of six equations.

114. Jacobi's memoir "Sur l'élimination des Nœuds \&c." (1843).-The problem of the motion of three mutually attracting bodies is in the first instance reduced to that of the motion of two fictitious bodies (which may be considered as mutually attracting bodies, attracted by a fixed centre of force) $\left(^{1}\right)$. In fact, in the original problem the

1 This is the effect of Jacobi's reduction; but the explicit statement of the theorem, and actual replacement of the problem of the three bodies by that of the two bodies attracted to a fixed centre, is due to Bertrand (post, No. 117). 
centre of gravity of the three bodies moves uniformly in a right line, and it may without any real loss of generality be taken to be at rest; that is, if the $x$-coordinates of the three bodies are $\xi_{1}, \xi_{2}, \xi_{3}$, then $m_{1} \xi_{1}+m_{2} \xi_{2}+m_{3} \xi_{3}=0$, or $\xi_{1}, \xi_{2}, \xi_{3}$ may be taken to be linear functions of two quantities $x_{1}$ and $x_{2}$. And similarly for the $y$-coordinates and the $z$-coordinates respectively. And $\left(x_{1}, y_{1}, z_{1}\right),\left(x_{2}, y_{2}, z_{2}\right)$ may be regarded as the coordinates of two bodies revolving about a fixed centre of force. Hence representing the differential coefficients in regard to the time by $x_{1}^{\prime}$, \&c., and treating these as new variables, the equations of motion will assume the form

$$
\frac{d x_{1}}{x_{1}^{\prime}}=\frac{d y_{1}}{y_{1}^{\prime}}=\frac{d z_{1}}{z_{1}^{\prime}}=\frac{d x_{2}}{x_{2}^{\prime}}=\frac{d y_{2}}{y_{2}^{\prime}}=\frac{d z_{2}}{z_{2}^{\prime}}=\frac{d x_{1}^{\prime}}{X_{1}}=\frac{d y_{1}^{\prime}}{Y_{1}}=\frac{d z_{1}^{\prime}}{Z_{1}}=\frac{d x_{2}^{\prime}}{X_{2}}=\frac{d y_{2}^{\prime}}{Y_{2}}=\frac{d z_{2}^{\prime}}{Z_{2}}(=d t)
$$

where $X_{1}, Y_{1}, Z_{1}, X_{2}, Y_{2}, Z_{2}$ are forces capable of representation by means of a forcefunction $U$. This is a system of twelve equations; but since $X_{1}, Y_{1}, Z_{1}, X_{2}, Y_{2}, Z_{2}$ are independent of the time, we may omit the equation $(=d t)$, and treat the system as one of eleven equations between the variables $x_{1}, y_{1}, z_{1}, x_{2}, y_{2}, z_{2}, x_{1}^{\prime}, y_{1}^{\prime}, z_{1}^{\prime}, x_{2}^{\prime}, y_{2}^{\prime}, z_{2}^{\prime}$ : if this system were integrated, the determination of the time would then depend on a quadrature only. But for the system of eleven equations we have four integrals, viz., the integral of Vis Viva and the three integrals of areas, and the system is thus reducible to one of $(11-4=)$ seven equations. This preliminary transformation in Jacobi's memoir explains the remark that the problem, as it stood before him, depended on a system of seven equations.

115. Jacobi remarks that in the transformed problem the three integrals of areas show (1) that the intersection of the planes of the orbits of the two bodies lie in a fixed plane, the invariable plane of the system; (2) that the inclinations of the planes of the two orbits to this fixed plane, and the parameters of the two orbits considered as variable ellipses, are four elements any two of which rigorously determine the two others.

And then choosing for variables the inclinations of the two orbits to the invariable plane, the two radius vectors, the angles which they form with the intersection of the planes of the two orbits, and lastly the angle between this intersection (being as already mentioned a line in the invariable plane) with a fixed line in the invariable plane, he finds that the last-mentioned angle entirely disappears from the system of differential equations, and is determined after their integration by a quadrature. In this new form of the differential equations there is no trace of the nodes. The differential equations which determine the relative motion of the three bodies are reduced to five equations of the first order and one of the second order. The equations in question are the equations I. to VI. given at the end of the memoir. It is to be remarked that the differential $d t$ is not eliminated from these equations; the last of them is $\frac{d^{2}}{d t^{2}}\left(\mu r^{2}+\mu_{1} r_{1}^{2}\right)=2 U-2 h$; and if to reduce them to a system of equations of the first order we write $\frac{d}{d t}\left(\mu r^{2}+\mu_{1} r_{1}^{2}\right)=\theta$, and therefore $\frac{d \theta}{d t}=2 U-2 h$, the system may be presented in the form

$$
\frac{d u}{U}=\frac{d u_{1}}{U_{1}}=\frac{d i}{I}=\frac{d i_{1}}{I_{1}}=\frac{d r}{R}=\frac{d r_{1}}{R_{1}}=\frac{d \theta}{\Theta}(=d t)
$$


which if we do, and then omit the equation $(=d t)$, we have a system of six equations between the seven quantities $u, u_{1}, i, i_{1}, r, r_{1}, \theta$; when this is integrated, the equation $(=d t)$ gives the time by a quadrature; and finally, Jacobi's equation VII. $\left(d \Omega=\tan u \frac{d i}{\sin i}\right)$ gives by a quadrature the angle before referred to as disappearing from the system of equations I. to VI.

116. But when Jacobi says, "Par suite on a fait cinq intégrations. Les intégrales connues n'étant qu'au nombre de quatre, on pourra donc dire que l'on a fait une intégration de plus dans le système du monde. Je dis dans le système du monde puisque la même méthode s'applique à un nombre quelconque de corps," the language used is not, I think, quite accurate. It in fact appears from the memoir that it is only on the assumption of the integration of the system of six equations that, besides the integral of Vis Viva and the integrals of areas, the remaining two integrals are known; in fact, after, but not before, the system of the order six has been integrated, the time $t$ and the angle $\Omega$ are each of them given by a quadrature.

117. Bertrand's “Mémoire sur l'intégration des équations différentielles de la Mécanique" (1852).-I have spoken of this memoir in No. 56 of my former Report. The course of investigation is the inquiry as to the integrals, which, combined according to Poisson's theorem with the integral of Vis Viva or any other given integral, give rise to an illusory result. But as regards the application made to the problem of three bodies, it will be more convenient to state from a different point of view the conclusions arrived at: and I may mention that when the author says "Je parviens.. à réduire la question à l'intégration de six équations toutes du premier ordre, c'est-à-dire que j'effectue une intégration de plus que ne l'avait fait Jacobi," he seems to have overlooked that Jacobi's system of five equations of the first order and one of the second order really is, as above noticed, a system of the six equations with another equation which then gives the time by a quadrature, and that, at least as appears to me, he has not advanced the solution beyond the point to which it had been carried by Jacobi ( $\left.{ }^{1}\right)$.

118. Presenting Bertrand's results in the slightly different notation in which they are reproduced in Bour's memoir (post. No. 122), then if $(x, y, z),\left(x_{1}, y_{1}, z_{1}\right)$ are the coordinates of the two bodies (the problem actually considered being, as with Jacobi, that of the motion of two bodies about a fixed centre of force), and representing the functions $x^{2}+y^{2}+z^{2}, \quad x_{1}^{2}+y_{1}{ }^{2}+z_{1}^{2}, \quad m^{2}\left(x^{\prime 2}+y^{\prime 2}+z^{\prime 2}\right), \quad m_{1}{ }^{2}\left(x_{1}^{\prime 2}+y_{1}^{\prime 2}+z_{1}^{\prime 2}\right), \quad m\left(x x^{\prime}+y y^{\prime}+z z^{\prime}\right)$, $m_{1}\left(x_{1} x_{1}^{\prime}+y_{1} y_{1}^{\prime}+z_{1} z_{1}^{\prime}\right), \quad m\left(x_{1} x^{\prime}+y_{1} y^{\prime}+z_{1} z^{\prime}\right), \quad m_{1}\left(x x_{1}^{\prime}+y y_{1}^{\prime}+z z_{1}^{\prime}\right), \quad\left(x x_{1}+y y_{1}+z z_{1}\right)$, $m m_{1}\left(x^{\prime} x_{1}^{\prime}+y^{\prime} y_{1}^{\prime}+z^{\prime} z_{1}^{\prime}\right)$ by $u, u_{1}, v, v_{1}, w, w_{1}, r, r_{1}, q, s$ respectively, then the lastmentioned quantities are connected by a single geometrical relation, so that any one of them, say $s$, may be considered as a given function of the remaining nine. And the author in effect shows that the equations of motion give a system

$$
\frac{d u}{U}=\frac{d u_{1}}{U_{1}}=\frac{d v}{V}=\frac{d v_{1}}{V_{1}}=\frac{d w}{W}=\frac{d w_{1}}{W_{1}}=\frac{d r}{R}=\frac{d r_{1}}{R_{1}}=\frac{d q}{Q}=(d t),
$$

1 These remarks were communicated by me to M. Bertrand-see my letter "Sur l'intégration des équations différentielles de la Mécanique," Comptes Rendus (1863)-and, in the answer he kindly sent me, he agrees that they are correct. 
where $U, U_{1}, \&$ c. are functions of the quantities $u, u_{1}, v$, \&c. Omitting from the system the equation $(=d t)$, there are eight equations between nine quantities; but there are two known integrals, viz., the integral of Vis Viva and the integral of principal moment (or sum of the squares of the integrals of areas); that is to say, the system is really a system of six equations.

119. Painvin, "Recherche du dernier Multiplicateur \&c." (1854).-The author investigates the ultimate multiplier for two systems of differential equations:

$1^{\circ}$. The system of the equations I. to VI: in Jacobi's memoir "Sur l'élimination des Nœuds \&c." (antè, No. 114). Writing in the equations $\frac{d r}{d t}=r^{\prime}, \frac{d r_{1}}{d t}=r_{1}^{\prime}$, and treating $r^{\prime}, r_{1}^{\prime}$ as new variables, the system may be written in the form

$$
\frac{d u}{U}=\frac{d u_{1}}{U_{1}}=\frac{d i}{I}=\frac{d i_{1}}{I_{1}}=\frac{d r}{R}=\frac{d r_{1}}{R_{1}}=\frac{d r^{\prime}}{R^{\prime}}=\frac{d r_{1}^{\prime}}{R_{1}^{\prime}}(=d t),
$$

which, omitting the equation $(=d t)$, is a system of seven equations between eight variables; and it is for this form of the system that the value of $M$ is determined, the result obtained being the simple and elegant one, $M=\frac{\sin i \sin i_{1}}{\sin ^{2} I}$. The system of seven equations has an integral which is in fact the equation $\mathrm{V}$. of the system in Jacobi's form, so that it is really a system of six equations (antè, No. 115).

$2^{\circ}$. The system secondly discussed is Bertrand's system of nine equations (antè, No. 118). The multiplier $M$ is obtained under four different forms, $M=\frac{1}{\sqrt{B^{2}-A C}}=\frac{1}{\sqrt{\alpha \alpha_{1}}}=\frac{1}{A Z+B}=\frac{1}{m n}$ (I do not stop to explain the notation), the last of them being referred to as a result announced by M. Bertrand in his course. But it is shown by M. Bour in the memoir next referred to (post, No. 122), that the multiplier for the system in question can be obtained in a very much more simple manner, almost without calculation.

120. In connexion with Jacobi's theory of the elimination of the Nodes, I may refer to the investigations "Application to the Problem of three Bodies," Nos. 84 to 96 of Donkin's memoir "On a Class of Differential Equations \&c." Part II. The author remarks that his differential equations No. 93 afford an example of the so-called elimination of the Nodes, quite different however (in that they are merely transformations of the original differential equations of the problem without any integrations) from that effected by Jacobi.

121. It may be right to refer again in this place to the concluding part of $\S 28$ of Jacobi's memoir "Nova Theoria Multiplicatoris \&c." (antè, No. 92), as bearing on the problem of three bodies.

122. Bour's "Mémoire sur le Problème des Trois Corps" (1856).-The author remarks that Bertrand's system of equations have lost the remarkable form and the properties which characterize the ordinary equations for the solution of a dynamical 
problem. But by selecting eight new variables, functions of Bertrand's variables, the system may be brought back to the standard Hamiltonian form

$$
\frac{d q_{i}}{d t}=\frac{d H}{d p_{i}}, \frac{d p_{i}}{d t}=-\frac{d H}{d q_{i}},
$$

or to the form adopted by M. Bour, of a partial differential equation

$$
\Sigma\left(\frac{d H}{d q_{i}} \frac{d \zeta}{d p_{i}}-\frac{d H}{d p_{i}} \frac{d \zeta}{d q_{i}}\right)=0
$$

and guiding himself by a theorem in relation to canonical integrals obtained in his memoir of 1855 (see No. 66 of my former Report), he finds by a somewhat intricate analysis the expressions of the eight new variables $p_{1}, p_{2}, p_{3}, p_{4}, q_{1}, q_{2}, q_{3}, q_{4}$. The results ultimately obtained are of a very remarkable and interesting form, viz. $H=$ funct. $\left(p_{1}, p_{2}, p_{3}, p_{4}, q_{1}, q_{2}, q_{3}, q_{4}\right)$ is equal to the value it would have for motion in a plane, plus a term admitting of a simple geometrical interpretation; and he thus arrives at the following theorem as a résumé of the whole memoir, viz.,

"In order to integrate in the general case the problem of three bodies, it is sufficient to solve the case of motion in a plane, and then to take account of a disturbing function equal to the product of a constant depending on the areas by the sum of the moments of inertia of the bodies round a certain axis, divided by the square of the triangle formed by the three bodies."

123. It may be remarked that the only given integral of the system of eight equations is the integral of $V i s$ Viva, $H=$ const., and that using this equation to eliminate one of the variables, and omitting the equation $(=d t)$, we have, as in the solutions of Jacobi and Bertrand, a system of six equations between seven variables. As the equations are in the standard dynamical form, no investigation is needed of the multiplier $M$, which is given by Jacobi's general theory, and consequently when any five integrals of the six equations are given, the remaining integral can be obtained by a quadrature.

In the case of three bodies moving in a plane, the solution takes a very simple form, which is given in the concluding paragraph of the memoir.

\section{Transformation of Coordinates, Article Nos. 124 to 141.}

124. It may be convenient to remark at once that two sets of rectangular coordinates may be related to each other properly or improperly, viz., the axes to which they belong (considered as drawn from the origin in the positive directions) may be either capable, or else incapable, of being brought into coincidence. The latter relation, although of equal generality with the former one, may for the most part be disregarded; for by merely reversing the directions of the one set of axes, the improper is converted into the proper relation. 
125. In the memoir "Problema Algebraicum \&c." (1770) Euler proposes to himself the question "Invenire novem numeros ita in quadratum disponendos
$A, \quad B, \quad C$
$D, \quad E, \quad F$
$G, \quad H, I$

ut satisfiat duodecem sequentibus conditionibus \&c.", viz., substituting for $A, B, C, \& c$. the ordinary letters

the twelve conditions are

$$
\begin{aligned}
& \alpha, \beta, \gamma \text {, } \\
& \alpha^{\prime}, \beta^{\prime}, \gamma^{\prime} \text {, } \\
& \alpha^{\prime \prime}, \beta^{\prime \prime}, \gamma^{\prime \prime} \text {, }
\end{aligned}
$$

$$
\begin{array}{ll}
\alpha^{2}+\alpha^{\prime 2}+\alpha^{\prime \prime 2}=1, & \alpha \beta+\alpha^{\prime} \beta^{\prime}+\alpha^{\prime \prime} \beta^{\prime \prime}=0, \\
\beta^{2}+\beta^{\prime 2}+\beta^{\prime 2}=1, & \beta \gamma+\beta^{\prime} \gamma^{\prime}+\beta^{\prime \prime} \gamma^{\prime \prime}=0, \\
\gamma^{2}+\gamma^{\prime 2}+\gamma^{\prime \prime 2}=1, & \gamma \alpha+\gamma^{\prime} \alpha^{\prime}+\gamma^{\prime \prime} \alpha^{\prime \prime}=0, \\
\alpha^{2}+\beta^{2}+\gamma^{2}=1, & \alpha \alpha^{\prime}+\beta \beta^{\prime}+\gamma \gamma^{\prime}=0, \\
\alpha^{\prime 2}+\beta^{\prime 2}+\gamma^{\prime 2}=1, & \alpha^{\prime} \alpha^{\prime \prime}+\beta^{\prime} \beta^{\prime \prime}+\gamma^{\prime} \gamma^{\prime \prime}=0, \\
\alpha^{\prime \prime 2}+\beta^{\prime \prime 2}+\gamma^{\prime \prime 2}=1, & \alpha \alpha^{\prime \prime}+\beta^{\prime \prime} \beta+\gamma^{\prime \prime} \gamma=0 .
\end{array}
$$

And he remarks that this is in fact the problem of the transformation of coordinates, viz., if we have

$$
\begin{aligned}
& X=\alpha x+\beta y+\gamma z, \\
& Y=\alpha^{\prime} x+\beta^{\prime} y+\gamma^{\prime} z, \\
& Z=\alpha^{\prime \prime} x+\beta^{\prime \prime} y+\gamma^{\prime \prime} z,
\end{aligned}
$$

then the first equations are such as to give identically

$$
X^{2}+Y^{2}+Z^{2}=x^{2}+y^{2}+z^{2} .
$$

126. Assuming the first six equations, he shows by a direct analytical process that $\alpha^{2}=\left(\beta^{\prime} \gamma^{\prime \prime}-\beta^{\prime \prime} \gamma^{\prime}\right)^{2}$, or $\alpha= \pm\left(\beta^{\prime} \gamma^{\prime \prime}-\beta^{\prime \prime} \gamma^{\prime}\right)$; or taking the positive sign (for, as the numbers may be taken as well positively as negatively, there is nothing lost by doing so) $\alpha=\beta^{\prime} \gamma^{\prime \prime}-\beta^{\prime \prime} \gamma^{\prime}$, which gives the system

$$
\begin{array}{lll}
\alpha=\beta^{\prime} \gamma^{\prime \prime}-\beta^{\prime \prime} \gamma^{\prime}, & \beta=\gamma^{\prime} \alpha^{\prime \prime}-\gamma^{\prime \prime} \alpha^{\prime}, & \gamma=\alpha^{\prime} \beta^{\prime \prime}-\alpha^{\prime \prime} \beta^{\prime}, \\
\alpha^{\prime}=\beta^{\prime \prime} \gamma-\beta \gamma^{\prime \prime}, & \beta^{\prime}=\gamma^{\prime \prime} \alpha-\gamma \alpha^{\prime \prime}, & \gamma^{\prime}=\alpha^{\prime \prime} \beta-\alpha \beta^{\prime \prime}, \\
\alpha^{\prime \prime}=\beta \gamma^{\prime}-\beta^{\prime} \gamma, & \beta^{\prime \prime}=\gamma \alpha^{\prime}-\gamma^{\prime} \alpha, & \gamma^{\prime \prime}=\alpha \beta^{\prime}-\alpha^{\prime} \beta^{\prime},
\end{array}
$$

and from these he deduces the second system of six equations. The inverse system of equations

is not explicitly referred to.

$$
\begin{aligned}
& X=\alpha x+\alpha^{\prime} y+\alpha^{\prime \prime} z \\
& Y=\beta x+\beta^{\prime} y+\beta^{\prime \prime} z, \\
& Z=\gamma x+\gamma^{\prime} y+\gamma^{\prime \prime} z
\end{aligned}
$$

c. IV. 
127. He then satisfies the equations by means of trigonometrical substitutions, viz., assuming $\alpha=\cos \zeta$, then $\alpha^{\prime 2}+\alpha^{\prime \prime 2}=\sin ^{2} \zeta$, which is satisfied by $\alpha^{\prime}=\sin \zeta \cos \eta, \alpha^{\prime \prime}=\sin \zeta \sin \eta$, \&c., and he thus obtains for the coefficients a set of values involving the angles $\zeta, \eta, \theta$, which are the same as those mentioned post, No. 130. And he shows how these formulæ may be obtained geometrically by three successive transformations of two coordinates only. The remainder of the memoir relates to the analogous problem of the transformation of four or more coordinates.

128. I have analysed so much of Euler's memoir in order to show that it contains nearly the whole of the ordinary theory of the transformation of coordinates; the only addition required is the equation

$$
\left|\begin{array}{ccc}
\alpha, & \beta, & \gamma \\
\alpha^{\prime}, & \beta^{\prime}, & \gamma^{\prime} \\
\alpha^{\prime \prime}, & \beta^{\prime \prime}, & \gamma^{\prime \prime}
\end{array}\right|= \pm 1,
$$

where the sign + gives $\alpha=\beta^{\prime} \gamma^{\prime \prime}-\beta^{\prime \prime} \gamma^{\prime}$, \&c. (ut supra), but the sign - would give $\alpha=-\left(\beta^{\prime} \gamma^{\prime \prime}-\beta^{\prime \prime} \gamma^{\prime}\right), \& c$.

129. The distinction of the ambiguous sign is in fact the above-mentioned one of the proper and improper transformations; viz., for the sign + the two sets of axes can, for the sign - they cannot, be brought into coincidence: this very important remark was, I believe, first made by Jacobi in one of his early memoirs in Crelle's Journal, but I have lost the reference. As already mentioned, it is allowable to attend only to the proper transformation, and to consider the value of the determinant as being $=+1$; and this is in fact almost always done.

130. Euler's formulæ involving the three angles are those which are ordinarily made use of in the problem of rotation and the problems of physical astronomy generally.

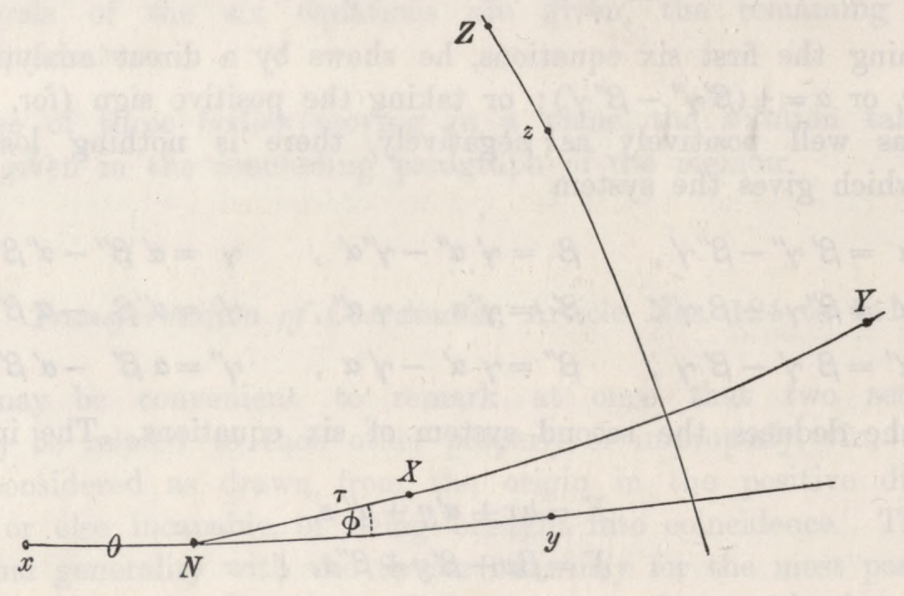

It is convenient to take them as in the figure, viz., $\theta$, the longitude of node, 
$\phi$, the inclination, $\tau$, the angular distance of $X$ from node, and the formulæ of transformation then are

\begin{tabular}{|c|c|c|c|}
\hline & $X$ & $Y$ & $Z$ \\
\hline$x$ & $\cos \tau \cos \theta-\sin \tau \sin \theta \cos \phi$ & $-\sin \tau \cos \theta-\cos \tau \sin \theta \cos \phi$ & $\sin \theta \sin \phi$ \\
\hline$y$ & $\frac{\cos \tau \sin \theta+\sin \tau \cos \theta \cos \phi}{-\sin \tau \sin \theta+\cos \tau \cos \theta \cos \phi}$ & $-\cos \theta \sin \phi$ \\
\hline$z$ & $\sin \tau \sin \phi$ & $\cos \tau \sin \phi$ & $\cos \phi$ \\
\hline
\end{tabular}

The foregoing very convenient algorithm, viz., the employment of

\begin{tabular}{|c|c|c|c|}
\hline & $X$ & $Y$ & $Z$ \\
\hline$x$ & $\alpha$ & $\beta$ & $\gamma$ \\
\hline$y$ & $\alpha^{\prime}$ & $\beta^{\prime}$ & $\gamma^{\prime}$ \\
\hline$z$ & $\alpha^{\prime \prime}$ & $\beta^{\prime \prime}$ & $\gamma^{\prime \prime}$ \\
\hline
\end{tabular}

to denote the system of equations

is due to M. Lamé.

$$
\begin{aligned}
& x=\alpha X+\beta Y+\gamma Z, \\
& y=\alpha^{\prime} X+\beta^{\prime} Y+\gamma^{\prime} Z, \\
& z=\alpha^{\prime \prime} X+\beta^{\prime \prime} Y+\gamma^{\prime \prime} Z
\end{aligned}
$$

131. But previously to the foregoing investigations, viz., in the memoir " $\mathrm{Du}$ Mouvement de Rotation \&c.," Mém. de Berlin for 1758 (pr. 1765), Euler had obtained incidentally a very elegant solution of the problem of the transformation of coordinates; this is in fact identical with the next mentioned one, the letters $l, m, n ; \lambda, \mu, \nu$ being used in the place of $\zeta, \zeta^{\prime}, \zeta^{\prime \prime} ; \eta, \eta^{\prime}, \eta^{\prime \prime}$.

132. In the memoir "Formulæ generales pro translatione \&c." (1775), Euler gives the following formulæ for the transformation of coordinates, viz., if the position of the set of axes $X Y Z$ in reference to the set $x y z$ is determined by

$$
x X, y X, z X=90^{\circ}-\zeta, 90^{\circ}-\zeta^{\prime}, 90^{\circ}-\zeta^{\prime \prime}, \angle^{8} Y X x, Y X y, Y X z=\eta, \eta^{\prime}, \eta^{\prime \prime},
$$

then the formulæ of transformation are

\begin{tabular}{|c|c|c|c|}
\hline & $\frac{X}{x}$ & $Y$ & $Z$ \\
\hline $\sin \zeta$ & $\cos \zeta \sin \eta$ & $\cos \zeta \cos \eta$ \\
\hline$y$ & $\sin \zeta^{\prime}$ & $\cos \zeta^{\prime} \sin \eta^{\prime}$ & $\cos \zeta^{\prime} \cos \eta^{\prime}$ \\
\hline$z$ & $\sin \zeta^{\prime \prime}$ & $\cos \zeta^{\prime \prime} \sin \eta^{\prime \prime}$ & $\cos \zeta^{\prime \prime} \cos \eta^{\prime \prime}$ \\
\hline
\end{tabular}


with the following equations connecting the six angles, viz., if

then

$$
-\Delta^{2}=\cos \left(\eta^{\prime}-\eta^{\prime \prime}\right) \cos \left(\eta^{\prime \prime}-\eta\right) \cos \left(\eta-\eta^{\prime}\right),
$$

$$
\tan \zeta=\frac{-\Delta}{\cos \left(\eta^{\prime}-\eta^{\prime \prime}\right)}, \quad \tan \zeta^{\prime}=\frac{-\Delta}{\cos \left(\eta^{\prime \prime}-\eta\right)}, \quad \tan \zeta^{\prime \prime}=\frac{-\Delta}{\cos \left(\eta-\eta^{\prime}\right)} .
$$

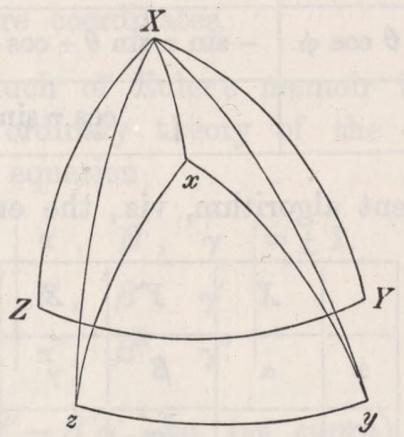

133. It is right to notice that these values of $\zeta, \zeta^{\prime}, \zeta^{\prime \prime}$ give the twelve equations $\alpha^{2}+\beta^{2}+\gamma^{2}=1$, \&c., but they do not give definitely $\alpha=\beta^{\prime} \gamma^{\prime \prime}-\beta^{\prime \prime} \gamma^{\prime}$, \&c., but only $\alpha= \pm\left(\beta^{\prime} \gamma^{\prime \prime}-\beta^{\prime \prime} \gamma^{\prime}\right)$; that is, in the formulæ in question the two sets of axes are not of necessity displacements the one of the other. In the same memoir Euler considers two sets of rectangular axes, and assuming that they are displacements the one of the other (this assumption is not made as explicitly as it should have been), he remarks that the one set may be made to coincide with the other set by means of a finite rotation about a certain axis (which may conveniently be termed the Resultant Axis). This consideration leads him to an equation which ought to be satisfied by the coefficients of transformation, but which he is not able to verify by means of the foregoing expressions in terms of $\zeta, \zeta^{\prime}, \zeta^{\prime \prime}, \eta, \eta^{\prime}, \eta^{\prime \prime}$.

134. I remark that Euler's equation in fact is

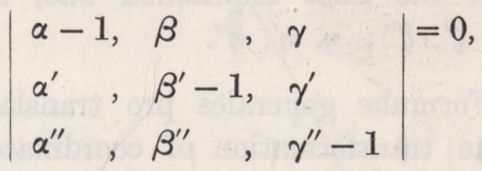

or, as it may be written,

$$
\left|\begin{array}{lll}
\alpha, & \beta, & \gamma \\
\alpha^{\prime}, & \beta^{\prime}, & \gamma^{\prime} \\
\alpha^{\prime \prime}, & \beta^{\prime \prime}, & \gamma^{\prime \prime}
\end{array}\right|-\left(\beta^{\prime} \gamma^{\prime \prime}-\beta^{\prime \prime} \gamma^{\prime}\right)-\left(\gamma^{\prime \prime} \alpha-\gamma \alpha^{\prime \prime}\right)-\left(\alpha \beta^{\prime}-\alpha^{\prime} \beta\right)+\alpha+\beta^{\prime}+\gamma^{\prime \prime}-1=0,
$$

in which form it is an immediate consequence of the equations

$$
\left|\begin{array}{lll}
\alpha, & \beta, & \gamma \\
\alpha^{\prime}, & \beta^{\prime}, & \gamma^{\prime} \\
\alpha^{\prime \prime}, & \beta^{\prime \prime}, & \gamma^{\prime}
\end{array}\right|=1, \alpha=\beta^{\prime} \gamma^{\prime \prime}-\beta^{\prime \prime} \gamma^{\prime}, \& c
$$

which are true for the proper, but not for the improper transformation. 
135. In the undated addition to the memoir, Euler states the theorem of the resultant axis as follows:- "Theorema. Quomodocunque sphæra circa centrum suum convertatur, semper assignari potest diameter cujus directio in situ translato conveniat cum situ originali;" and he again endeavours to obtain a verification of the foregoing analytical theorem.

136. The theory of the Resultant Axis was further developed by Euler in the memoir "Nova Methodus Motum \&c." (1775), and by Lexell in the memoir "Nonnulla theoremata generalia \&c." (1775): the geometrical investigations are given more completely and in greater detail in Lexell's memoir. The result is contained in the following system of formulæ for the transformation of coordinates, viz., if $\alpha, \beta, \gamma$ are the inclinations of the resultant axis to the original set, and if $\phi$ is the rotation about the resultant axis, or say the resultant rotation, then we have

\begin{tabular}{|c|c|c|c|}
\hline & $X$ & $Y$ & $Z$ \\
\hline$x$ & $\cos ^{2} \alpha+\sin ^{2} \alpha \cos \phi$ & $\cos \alpha \cos \beta(1-\cos \phi)+\cos \gamma \sin \phi$ & $\cos \alpha \cos \gamma(1-\cos \phi)-\cos \beta \sin \phi$ \\
\hline$y$ & $\cos \beta \cos \alpha(1-\cos \phi)-\cos \gamma \sin \phi$ & $\cos ^{2} \beta+\sin ^{2} \beta \cos \phi$ & $\cos \beta \cos \gamma(1-\cos \phi)+\cos \alpha \sin \phi$ \\
\hline$z$ & $\cos \gamma \cos \alpha(1-\cos \phi)+\cos \beta \sin \phi$ & $\cos \gamma \cos \beta(1-\cos \phi)-\cos \alpha \sin \phi$ & $\cos ^{2} \gamma+\sin ^{2} \gamma \cos \phi$ \\
\hline
\end{tabular}

Euler attempts, but not very successfully, to apply the formulæ to the dynamical problem of the rotation of a solid body: he does not introduce them into the differential equations, but only into the integral ones, and his results are complicated and inelegant. The further simplification effected by Rodrigues was in fact required.

137. Jacobi's paper, “Euleri formulæ \&c." (1827), merely cites the last-mentioned result.

138. I find it stated in Lacroix's Differential Calculus, t. I. p. 533, that the following system for the transformation of coordinates was obtained by Monge (no reference is given in Lacroix), viz., the system being as above,

$$
\begin{array}{lll}
\alpha, & \beta, & \gamma, \\
\alpha^{\prime}, & \beta^{\prime}, & \gamma^{\prime}, \\
\alpha^{\prime \prime}, & \beta^{\prime \prime}, & \gamma^{\prime \prime},
\end{array}
$$

and the quantities $\alpha, \beta^{\prime}, \gamma^{\prime \prime}$ being arbitrary, then putting

so that

$$
\begin{aligned}
& 1+\alpha+\beta^{\prime}+\gamma^{\prime \prime}=M, \\
& 1+\alpha-\beta^{\prime}-\gamma^{\prime \prime}=N, \\
& 1-\alpha+\beta^{\prime}-\gamma^{\prime \prime}=P, \\
& 1-\alpha-\beta^{\prime}+\gamma^{\prime \prime}=Q,
\end{aligned}
$$

$$
M+N+P+Q=4,
$$


we have

$$
\begin{array}{lll}
2 \beta=\sqrt{N Y}+\sqrt{M Q}, & 2 \gamma^{\prime}=\sqrt{P Q}+\sqrt{M N}, & 2 \alpha^{\prime \prime}=\sqrt{Q N}+\sqrt{M P}, \\
2 \alpha^{\prime}=\sqrt{N P}-\sqrt{M Q}, & 2 \beta^{\prime \prime}=\sqrt{P Q}-\sqrt{M N}, & 2 \gamma=\sqrt{Q N}-\sqrt{M P} .
\end{array}
$$

These are formulæ very closely connected with those of Rodrigues.

139. The theory was perfected by Rodrigues in the valuable memoir "Des lois géométriques \&c." (1840). Using for greater convenience $\lambda, \mu_{2} \nu$ in the place of his $\frac{1}{2} m, \frac{1}{2} n, \frac{1}{2} p$, he in effect writes

$$
\begin{aligned}
& \tan \frac{1}{2} \phi \cos \alpha=\lambda, \\
& \tan \frac{1}{2} \phi \cos \beta=\mu, \\
& \tan \frac{1}{2} \phi \cos \gamma=\nu,
\end{aligned}
$$

and this being so, the coefficients of transformation are

$$
\begin{array}{lll}
1+\lambda^{2}-\mu^{2}-\nu^{2}, & 2(\lambda \mu+\nu) \quad, & 2(\lambda \nu-\mu) \\
2(\mu \lambda-\nu) \quad, & 1-\lambda^{2}+\mu^{2}-\nu^{2}, & 2(\mu \nu+\lambda) \\
2(\nu \lambda+\mu) \quad, & 2 \nu \mu-\lambda \quad, & 1-\lambda^{2}-\mu^{2}+\nu^{2},
\end{array}
$$

all divided by the common denominator $1+\lambda^{2}+\mu^{2}+\nu^{2}$. Conversely, if the coefficients of transformation are as usual represented by

$$
\begin{array}{lll}
\alpha, & \beta, & \gamma, \\
\alpha^{\prime}, & \beta^{\prime}, & \gamma^{\prime}, \\
\alpha^{\prime \prime}, & \beta^{\prime \prime}, & \gamma^{\prime \prime},
\end{array}
$$

then $\lambda^{2}, \mu^{2}, \nu^{2}, \lambda, \mu, \nu$ are respectively equal to

$$
\begin{array}{lll}
1+\alpha-\beta^{\prime}-\gamma^{\prime \prime}, & 1-\alpha+\beta^{\prime}-\gamma^{\prime \prime}, & 1=\alpha-\beta^{\prime}+\gamma^{\prime \prime}, \\
\gamma^{\prime}-\beta^{\prime \prime} \quad, & \alpha^{\prime \prime}-\beta \quad, \quad \beta-\alpha^{\prime}
\end{array}
$$

each of them divided by $1+\alpha+\beta^{\prime}+\gamma^{\prime \prime}$.

The memoir contains very elegant formulæ for the composition of finite rotations, and it will be again referred to in speaking of the kinematics of a solid body.

140. Sir W. R. Hamilton's first papers on the theory of quaternions were published in the years 1843 and 1844: the fundamental idea consists in the employment of the imaginaries $i, j, k$, which are such that

whence also

$$
\begin{gathered}
i^{2}=j^{2}=k^{2}=-1, \quad j k=-k j=i, \quad k i=-i k=j, \quad i j=-j i=k, \\
(w+i x+j y+k z)\left(w^{\prime}+i x^{\prime}+j y^{\prime}+k z^{\prime}\right) \\
=\quad w w^{\prime}-x x^{\prime}-y y^{\prime}-z z^{\prime} \\
+i\left(w x^{\prime}+w^{\prime} x+y z^{\prime}-y^{\prime} z\right) \\
+j\left(w y^{\prime}+w^{\prime} y+z x^{\prime}-z^{\prime} x\right) \\
+k\left(w z^{\prime}+w^{\prime} z+x y^{\prime}-x y\right)
\end{gathered}
$$


so that representing the right-hand side by

we have identically

$$
W+i X+j Y+k Z
$$

$$
W^{2}+X^{2}+Y^{2}+Z^{2}=\left(w^{2}+x^{2}+y^{2}+z^{2}\right)\left(w^{\prime 2}+x^{\prime 2}+y^{\prime 2}+z^{\prime 2}\right) .
$$

It is hardly necessary to remark that Sir W. R. Hamilton in his various publications on the subject, and in the Lectures on Quaternions, Dublin, 1853, has developed the theory in detail, and has made the most interesting applications of it to geometrical and dynamical questions; and although the first explicit application of it to the present question may have been made in my own paper next referred to, it seems clear that the whole theory was in its original conception intimately connected with the notion of rotation.

141. Cayley, "On certain Results relating to Quaternions" (1845).-It is shown that Rodrigues' transformation formula may be expressed in a very simple manner by means of quaternions; viz., we have

$$
i x+j y+k z=(1+i \lambda+j \mu+k \nu)^{-1}(i X+j Y+k Z)(1+i \lambda+j \mu+k \nu),
$$

where developing the function on the right-hand side, and equating the coefficients of $i, j, k$, we obtain the formulæ in question. A subsequent paper, Cayley, "On the application of Quaternions to the Theory of Rotation" (1848), relates to the composition of rotations.

Principal Axes, and Moments of Inertia. Article Nos. 142-163.

142. The theorem of principal axes consists herein, that at any point of a solid body there exists a system of axes $\mathrm{Ox}, \mathrm{Oy}, \mathrm{Oz}$, such that

$$
\int y z d m=0, \quad \int z x d m=0, \quad \int x y d m=0 .
$$

But this, the original form of the theorem, is a mere deduction from a general theory of the representation of the integrals

$$
\int x^{2} d m, \int y^{2} d m, \int z^{2} d m, \int y z d m, \int z x d m, \int x y d m
$$

for any axes through the given origin by means of an ellipsoid depending on the values of these integrals corresponding to a given set of rectangular axes through the same origin.

143. If, for convenience, we write as follows, $M=\int d m$ the mass of the body, and

$$
A^{\prime}=\int x^{2} d m, \quad B^{\prime}=\int y^{2} d m, \quad C^{\prime}=\int z^{2} d m, \quad F^{\prime}=\int y z d m, \cdot G^{\prime}=\int z x d m, \quad H^{\prime}=\int x y d m,
$$


and moreover

$$
\begin{gathered}
A=\int\left(y^{2}+z^{2}\right) d m, \quad B=\int\left(z^{2}+x^{2}\right) d m, \quad C=\int\left(x^{2}+y^{2}\right) d m \\
F=-\int y z d m, \quad G=-\int z x d m, \quad H=-\int x y d m,\left({ }^{1}\right)
\end{gathered}
$$

so that

$$
A=B^{\prime}+C^{\prime}, \quad B=C^{\prime}+A^{\prime}, \quad C=A^{\prime}+B^{\prime}, \quad F=-F^{\prime}, \quad G=-G^{\prime}, \quad H=-H^{\prime},
$$

then the ellipsoid which in the first instance presents itself for this purpose, and which Prof. Price has termed the Ellipsoid of Principal Axes, but which I would rather term the "Comomental Ellipsoid," is the ellipsoid

$$
\left(A^{\prime}, B^{\prime}, C^{\prime}, F^{\prime}, G^{\prime}, H^{\prime} \chi x, y, z\right)^{2}=M k^{4},
$$

where $k$ is arbitrary, so that the absolute magnitude is not determined. But it is more usual, and in some respects better to consider in place thereof the "Momental Ellipsoid" (Cauchy, "Sur les Moments d'Inertie," Exercices de Mathématique, t. II. pp. $93-103,1827)$,

$$
\left(A, B, C, F, G, H \gamma(x, y, z)^{2}=M k^{4},\right.
$$

or as it may also be written,

$$
\left(A^{\prime}+B^{\prime}+C^{\prime}\right)\left(x^{2}+y^{2}+z^{2}\right)-\left(A^{\prime}, B^{\prime}, C^{\prime}, F^{\prime}, G^{\prime}, H^{\prime} \gamma x, y, z\right)^{2}=M k^{4},
$$

which shows that the two ellipsoids have their axes, and also their circular sections, coincident in direction.

144. And there is besides this a third ellipsoid, the "Ellipsoid of Gyration," which is the reciprocal of the momental ellipsoid in regard to the concentric sphere, radius $k$. The last-mentioned ellipsoid is given in magnitude, viz., if the body is referred to its principal axes, then putting $A=M a^{2}, B=M b^{2}, C=M c^{2}$, the equation of the ellipsoid of gyration is

$$
\frac{x^{2}}{a^{2}}+\frac{y^{2}}{b^{2}}+\frac{z^{2}}{c^{2}}=1
$$

The axes of any one of the foregoing ellipsoids coincide in direction with the principal axes of the body, and the magnitudes of the axes lead very simply to the values of the principal moments $A, B, C$.

145. The origin has so far been left arbitrary: in the dynamical applications, this origin is in the case of a solid body rotating about a fixed point, the fixed point; and in the case of a free body, the centre of gravity. But the values of the coefficients $(A, B, C, F, G, H)$, or $\left(A^{\prime}, B^{\prime}, C^{\prime}, F^{\prime}, G^{\prime}, H^{\prime}\right)$, corresponding to any given origin whatever, are very easily expressed in terms of the coordinates of this origin, and the values of the corresponding coefficients for the centre of gravity as origin; or, what is the same thing, any one of the ellipsoids for the given origin may be geometrically con-

1 I have ventured to make this change instead of writing as usual $F=\int y z d m$, \&c.; as in most cases $F=G=H=0$, the formulæ affected by the alteration are not numerous. 
structed by means of the ellipsoid for thè centre of gravity. The geometrical theory, as regards the magnitudes of the axes, does not appear to have been anywhere explicitly enunciated; as regards their direction, it is comprised in the theorem that the directions at any point are the three rectangular directions at that point in regard to the ellipsoid of gyration for the centre of gravity $\left({ }^{1}\right)$, post, No. 159. The notion of the ellipsoids, and of the relation between the ellipsoids at a given point and those at the centre of gravity, once established, the theory of principal axes and moments of inertia becomes a purely geometrical one.

146. The existence of principal axes was. first established by Segner in the work Specimen Theorice Turbinum, Halle (1755), where, however, it is remarked that Euler had said something on the subject in the \{Berlin\} Memoirs for 1749 and 1750 (post, No. 167 ), and had constructed a new mechanical principle, but without pursuing the question. Segner's course of investigation is in principle the same as that now made use of, viz. a principal axis is defined to be an axis such that when a body revolves round it the forces arising from the rotation have no tendency to alter the position of the axes. It is first shown that there are systems of axes $x, y, z$ such that $\int x z d m=0$, and then, in reference to such a set of axes, the position of a principal axis, say the axis of $X$, is determined by the conditions $\int X Y d m=0, \int X \boldsymbol{Z} d m=0$, viz. the unknown quantities being taken to be $t=\frac{\cos \alpha}{\cos \gamma}, \tau=\frac{\cos \beta}{\cos \gamma}(\alpha, \beta, \gamma$ being the inclinations of the principal axis to those of $x, y, z)$, and then putting $A=\int x^{2} d m$, \&c. ( $F=0$ by hypothesis), Segner's equations for the determination of $t, \tau$, are

the second of which gives

$$
\begin{aligned}
& G^{\prime} t^{2}+\left(C^{\prime}-A^{\prime}\right) t-G^{\prime}-H^{\prime} \tau=0, \\
& \left(C^{\prime}-B^{\prime}\right) \tau-G^{\prime} t \tau+H^{\prime} t=0,
\end{aligned}
$$

$$
\tau=\frac{H^{\prime} t}{B^{\prime}-C^{\prime}+G^{\prime} t}
$$

and by means of it the first gives

$$
G^{\prime 2} t^{3}-G^{\prime}\left(A^{\prime}-B^{\prime}\right) t^{2}+\left\{\left(B^{\prime}-C^{\prime}\right)\left(C^{\prime}-A^{\prime}\right)-G^{\prime 2}-H^{2}\right\} t+G^{\prime}\left(B^{\prime}-C^{\prime}\right)=0,
$$

which being a cubic equation shows that there are three principal axes; and it is afterwards proved that these are at right angles to each other.

147. To show the equivalence of Segner's solution to the modern one, I remark that if $u=\int X^{2} d m$, we have

$$
\begin{array}{rrr}
\left(A^{\prime}-u\right) t+H^{\prime} & \tau+G^{\prime} & =0 \\
B^{\prime} \quad t+\left(B^{\prime}-u\right) \tau+F^{\prime \prime} & =0 \\
G^{\prime} \quad t+F^{\prime} & \tau+C^{\prime}-u= & 0
\end{array}
$$

1 The rectangular directions at a point in regard to an ellipsoid are the directions of the axes of the circumscribed cone, or, what is the same thing, they are the directions of the normals to the three quadric surfaces, confocal with the given ellipsoid, which pass through the given point. The theory of confocal surfaces appears to have been first given by Chasles, Note XXXI. of the Aperçu Historique (1837).

C. IV. 
whence

$$
\begin{aligned}
t^{2}: \tau^{2}: 1: \tau: t: t \tau= & B^{\prime} C^{\prime}-F^{\prime 2}-\left(B^{\prime}+C^{\prime}\right) u+u^{2} \\
& : C^{\prime} A^{\prime}-G^{\prime 2}-\left(C^{\prime}+A^{\prime}\right) u+u^{2} \\
& : A^{\prime} B^{\prime}-H^{\prime 2}-\left(A^{\prime}+B^{\prime}\right) u+u^{2} \\
& : G^{\prime} H^{\prime}-A^{\prime} F^{\prime}+F^{\prime \prime} u \\
& : H^{\prime} F^{\prime \prime}-B^{\prime} G^{\prime}+G^{\prime} u \\
& : F^{\prime \prime} G^{\prime}-C^{\prime} H^{\prime}+H^{\prime} u,
\end{aligned}
$$

or putting therein $F^{\prime}=0$,

$$
\begin{aligned}
t^{2}: \tau^{2}: 1: \tau: t: t \tau= & B^{\prime} C^{\prime}-\left(B^{\prime}+C^{\prime}\right) u+u^{2} \\
& : C^{\prime} A^{\prime}-G^{\prime 2}-\left(C^{\prime}+A^{\prime}\right) u+u^{2} \\
& : A^{\prime} B^{\prime}-H^{\prime 2}-\left(A^{\prime}+B^{\prime}\right) u+u^{2} \\
& : G^{\prime} H^{\prime} \\
& :-B^{\prime} G^{\prime} \\
& :-C^{\prime} H^{\prime}
\end{aligned}
$$

by means of which Segner's equations may be verified. I have given this analysis, as the first solution of such a problem is a matter of interest.

148. There is little if anything added to Segner's results by the memoir, Euler, "Recherches sur la Connaissance Mécanique des Corps" (1758), which is introductory to the immediately following one on Rotation.

149. Relating to the theory of principal axes we have Binet's "Mémoire sur les axes conjugués \&c.", (1813). The author proposes to make known the new systems of axes which he calls conjugate axes, which, when they are at right angles to each other, coincide with the principal axes; viz. considering the sum of the molecules each into its distance from a plane, such distance being measured in the direction of a line, then (the direction of the line being given) of all the planes which pass through a given point, there is one for which the sum in question is a minimum, and this plane is said to be conjugate to the given line, and from the notion of a line and conjugate plane he passes to that of a system of conjugate axes. The investigation (which is throughout an elegant one) is conducted analytically; the coordinates made use of are oblique ones, and the formulæ are thus rendered more complicated than they would have been; in referring to them it will be convenient to make the axes rectangular.

150. One of the results is the well-known equation

$$
\left(A^{\prime}-\Theta\right)\left(B^{\prime}-\Theta\right)\left(C^{\prime}-\Theta\right)-F^{\prime 2}\left(A^{\prime}-\Theta\right)-G^{\prime 2}\left(B^{\prime}-\Theta\right)-H^{\prime 2}\left(C^{\prime}-\Theta\right)+2 F^{\prime} G^{\prime} H^{\prime}=0
$$

which, if $x_{1}, y_{1}, z_{1}$ are the principal axes, has for its roots $\int x_{1}^{2} d m, \int y_{1}^{2} d m, \int z_{1}^{2} d m$. 
And the equations (1), p. 49, taking therein the original axes as rectangular, are

$$
\begin{array}{llll} 
& \left(\mathfrak{H}^{\prime}-\frac{K^{\prime}}{\Theta^{\prime}}\right) \cos \alpha+\mathfrak{S}^{\prime} & \cos \beta+\mathscr{F}^{\prime} & \cos \gamma=0, \\
+\mathfrak{S}^{\prime} & \cos \alpha+\left(\mathfrak{B}^{\prime}-\frac{K^{\prime}}{\Theta^{\prime}}\right) \cos \beta+\mathscr{F}^{\prime} & \cos \gamma=0, \\
+\mathfrak{F}^{\prime} & \cos \alpha+\mathscr{F}^{\prime} & \cos \beta+\left(\mathfrak{S}^{\prime}-\frac{K^{\prime}}{\Theta^{\prime}}\right) \cos \gamma=0,
\end{array}
$$

where $\mathfrak{I}^{\prime}, \mathfrak{B}^{\prime}, \mathfrak{C}^{\prime}, \mathfrak{F}^{\prime}, \mathfrak{F}^{\prime}, \mathfrak{S}^{\prime}$ denote the reciprocal coefficients, $\mathfrak{F}^{\prime}=B^{\prime} C^{\prime}-F^{\prime 2}$ \&c., and $K^{\prime}$ is the discriminant $=A^{\prime} B^{\prime} C^{\prime}-A^{\prime} F^{\prime 2}-B^{\prime} G^{\prime 2}-C^{\prime} H^{\prime 2}+2 F^{\prime} G^{\prime} H^{\prime}$ : this is a symmetrical system of equations for finding $\cos \alpha: \cos \beta: \cos \gamma$, less simple however than the modern form (post, No. 154), the identity of which with Binet's may be shown without difficulty.

151. Another result (p. 57) is that if the original axes are principal axes, and if $O x, O y, O z$ are the principal axes through a point the coordinates whereof are $f, g, h$, and if $\Theta_{1}^{\prime}=$ (say) $\int x_{1}^{2} d m$, then we have

$$
\frac{f^{2}}{\Theta_{1}^{\prime}-A^{\prime}}+\frac{g^{2}}{\Theta_{1}^{\prime}-B^{\prime}}+\frac{h^{2}}{\Theta_{1}^{\prime}-C^{\prime}}=\frac{1}{M},
$$

(in which $I$ have restored the mass $M$, which is put equal to unity), so that if $\Theta_{1}^{\prime}$ have a given constant value, the locus of the point is a quadric surface, the nature whereof will depend on the value of $\Theta_{1}$. The surfaces in question are confocal with each other \{and with the imaginary surface $\frac{x^{2}}{-A^{\prime}}+\frac{y^{2}}{-B^{\prime}}+\frac{z^{2}}{-C^{\prime}}=\frac{1}{M}$, which is similar to the ellipsoid $\frac{x^{2}}{A^{\prime}}+\frac{y^{2}}{B^{\prime}}+\frac{z^{2}}{C^{\prime}}=\frac{1}{M}$, which is the reciprocal of the comomental ellipsoid $A^{\prime} x^{2}+B^{\prime} y^{2}+C^{\prime} z^{2}=M k^{2}$ in regard to a concentric sphere, radius $k$ \}. The author mentions the ellipsoid $\frac{x^{2}}{A^{\prime}}+\frac{y^{2}}{B^{\prime}}+\frac{z^{2}}{C^{\prime}}=\frac{1}{M}$ (see p. 64 ), and he remarks that his conjugate axes are in fact conjugate axes in respect to this ellipsoid, and consequently that the principal axes are in direction the principal axes of this ellipsoid: it is noticeable that the ellipsoid thus incidentally considered is not the comomental ellipsoid itself, but, as just remarked, its reciprocal in regard to a concentric sphere.

152. Poisson, Mécanique (1st ed. 1811, and indeed 2nd ed. 1833), gives the theory of principal axes in a less complete form than in Binet's memoir; for the directions of the principal axes are obtained in anything but an elegant form.

153. Ampère's Memoir (1823).-The expression permanent axis is used in the place of principal axis, which is employed to designate a principal axis through the centre of gravity. The memoir contains a variety of very interesting geometrical theorems, which however, as no ellipsoid is made use of, can hardly be considered as exhibited in their proper connexion. The author arrives incidentally at certain conics, which are in fact the focal conics of the ellipsoid of gyration $\left(\frac{x^{2}}{A}+\frac{y^{2}}{B}+\frac{z^{2}}{C}=\frac{1}{M}\right)$ for the centre of gravity. 
154. Cauchy, in the memoir "Sur les Momens d'Inertie" (1827), considers the momental ellipsoid $\left(A, B, C, F, G, H \gamma(x, y, z)^{2}=1\right.$, and employs it as well to prove the existence of the principal axes as to determine their direction, and also the magnitudes of the principal moments; the results are obtained in the simplest and best forms; viz. the direction cosines are given by

$$
\begin{array}{llll}
(A-\Theta) \cos \alpha+H & \cos \beta+G & \cos \gamma=0, \\
H & \cos \alpha+(B-\Theta) \cos \beta+F & \cos \gamma=0, \\
G & \cos \alpha+F & \cos \beta+(C-\Theta) \cos \gamma=0,
\end{array}
$$

where

$$
(A-\Theta)(B-\Theta)(C-\Theta)-(A-\Theta) F^{2}-(B-\Theta) G^{2}-(C-\Theta) H^{2}+2 F G H=0,
$$

$\Theta$ being one of the principal moments.

155. Poinsot, "Mémoire sur la Rotation" (1834), defines the "Central Ellipsoid" as an ellipsoid having for its axes the principal axes through the centre of gravity, the squares of the lengths being reciprocally proportional to the principal moments; and he remarks in passing that the moment about any diameter of the ellipsoid is inversely proportional to the square of this diameter. It is to be noticed that Poinsot speaks only of the ellipsoid having its centre at the centre of gravity, but that such ellipsoid may be constructed about any point whatever as centre, and that so generalized it is in fact the momental ellipsoid $A x^{2}+B y^{2}+C z^{2}=M k^{4}$; and moreover that Poinsot defines his ellipsoid by reference to the principal axes.

156. Pirie, "On the Principal Axes \&c." (1837), obtained analytically in a very elegant manner equations for determining the positions of the principal axes; viz. these are

where

$$
\begin{array}{lll}
\left(A^{\prime}-\Theta^{\prime}\right) \cos \alpha+H^{\prime} & \cos \beta+G^{\prime} & \cos \gamma=0, \\
H^{\prime} & \cos \alpha+\left(B^{\prime}-\Theta^{\prime}\right) \cos \beta+F^{\prime} & \cos \gamma=0, \\
G^{\prime} & \cos \alpha+F^{\prime \prime} & \cos \beta+\left(C^{\prime}-\Theta^{\prime}\right) \cos \gamma=0,
\end{array}
$$

$$
\left(A^{\prime}-\Theta^{\prime}\right)\left(B^{\prime}-\Theta^{\prime}\right)\left(C^{\prime}-\Theta^{\prime}\right)-\left(A^{\prime}-\Theta^{\prime}\right) F^{\prime 2}-\left(B^{\prime}-\Theta^{\prime}\right) G^{\prime 2}-\left(C^{\prime}-\Theta^{\prime}\right) F^{\prime 2}+2 F^{\prime} G^{\prime} H^{\prime}=0 ;
$$

viz. these are similar to those of Cauchy, only they belong to the comomental instead of the momental ellipsoid.

157. Maccullagh, in his Lectures of 1844 (see Haughton), considers the momental ellipsoid

$$
(A, B, C, F, G, H \chi x, y, z)^{2}=M k^{4}
$$

( $A, B, C, F, G, H$ ut supra), which is such that the moment of inertia of the body with respect to any axis passing through the origin is proportional to the square of the radius vector of the ellipsoid; and from the geometrical theorem of the ellipsoid having principal axes he obtained the mechanical theorem of the existence of principal axes of the body; at least I infer that he did so, although the conclusion is not 
explicitly stated in Haughton's account; but in all this he had been anticipated by Cauchy. And afterwards, referring the ellipsoid to its principal axes, so that the equation is $A x^{2}+B y^{2}+C z^{2}=M k^{4}$, he writes $A=M a^{2}, B=M b^{2}, C=M c^{2}$, which reduces the equation to $a^{2} x^{2}+b^{2} y^{2}+c^{2} z^{2}=k^{4}$, and he considers the reciprocal ellipsoid $\frac{x^{2}}{a^{2}}+\frac{y^{2}}{b^{2}}+\frac{z^{2}}{c^{2}}=1$, or, what is the same thing, $\frac{x^{2}}{A}+\frac{y^{2}}{B}+\frac{z^{2}}{C}=\frac{1}{M}$, which is the ellipsoid of gyration.

158. Thomson, "On the Principal Axes of a Solid Body" (1846), shows analytically that the principal axes coincide in direction with the axes of the momental ellipsoid

$$
\left(A, B, C, F, G, H \gamma(x, y, z)^{2}=M k^{4} ;\right.
$$

but the geometrical theorem might have been assumed: the investigation is really an investigation of the axes of this ellipsoid. And he remarks that the ellipsoid $\left(A^{\prime}, B^{\prime}, C^{\prime}, F^{\prime}, G^{\prime}, H^{\prime} \gamma(x, y, z)^{2}=M k^{4}\right.$ (the comomental ellipsoid) might equally well have been used for the purpose.

159. He obtains the before-mentioned theorem that the directions of the principal axes at any point are the rectangular directions in regard to the ellipsoid of gyration $\left(\frac{x^{2}}{A}+\frac{y^{2}}{B}+\frac{z^{2}}{C}=\frac{1}{M}\right)$ for the centre of gravity. And for determining the moments of inertia at the given point (say its coordinates are $\xi, \eta, \zeta$ ) he obtains the equation

$$
\frac{\xi^{2}}{\xi^{2}+\eta^{2}+\zeta^{2}+\frac{A-P}{M}}+\frac{\eta^{2}}{\xi^{2}+\eta^{2}+\zeta^{2}+\frac{B-P}{M}}+\frac{\zeta^{2}}{\xi^{2}+\eta^{2}+\zeta^{2}+\frac{C-P}{M}}=1
$$

where the three roots of the cubic in $P$ are the required moments. Analytically nothing can be more elegant, but, as already remarked, a geometrical construction for the magnitudes of these moments appears to be required.

160. Some very interesting geometrical results are obtained by considering the "equimomental surface", the locus of the points for which one of the moments of inertia is equal to a given quantity $\Pi$; the equation is of course

$$
\frac{x^{2}}{x^{2}+y^{2}+z^{2}+\frac{A-\Pi}{M}}+\frac{y^{2}}{x^{2}+y^{2}+z^{2}+\frac{B-\Pi}{M}}+\frac{z^{2}}{x^{2}+y^{2}+z^{2}+\frac{C-\Pi}{M}}=1,
$$

which includes Fresnel's wave-surface. In particular it is shown that the equimomental surface cuts any surface

$$
\frac{x^{2}}{A+\Theta}+\frac{y^{2}}{B+\Theta}+\frac{z^{2}}{C+\Theta}=\frac{1}{M}
$$

confocal with the ellipsoid of gyration in a spherical conic and a curve of curvature; a theorem which is also demonstrated, Cayley, "Note on a Geometrical Theorem \&c." (1846). 
161. Townsend, "On principal Axes \&c." (1846).-This elaborate paper is contemporaneous, or nearly so, with Thomson's, and several of the conclusions are common to the two. From the character of the paper, I find it difficult to give an account of it; and I remark that, the theory of principal axes once brought into connexion with that of confocal surfaces, all ulterior developments belong more properly to the latter theory.

162. Haton de la Goupillière's two memoirs, "Sur la Théorie Nouvelle de la Géométrie des Masses" (1858), relate in a great measure to the theory of the integral $\int x y d m$, and its variations according to the different positions of the two planes $x=0$ and $y=0$; the geometrical interpretations of the several results appear to be given with much care and completeness, but I have not examined them in detail. The author refers to the researches of Thomson and Townsend.

163. I had intended to show (but the paper has not been completed for publication) how the momental ellipsoid for any point of the body may be obtained from that for the centre of gravity by a construction depending on the "square potency" of a point in regard to the last-mentioned ellipsoid.

The Rotation of a solid body. Article Nos. 164-207.

164. It will be recollected that the problem is the same for a body rotating about a fixed point, and for the rotation of a free body about the centre of gravity; the case considered is that of a body not acted on by any forces. According to the ordinary analytical mode of treatment, the problem depends upon Euler's equations

$$
\begin{aligned}
& A d p+(C-B) q r d t=0, \\
& B d q+(A-C) r p d t=0, \\
& C d r+(B-A) p q d t=0,
\end{aligned}
$$

for the determination of $p, q, r$, the angular velocities about the principal axes; considering $p, q, r$ as known, we obtain by merely geometrical considerations a system of three differential equations of the first order for the determination of the position in space of the principal axes.

165. The solution of these, which constitutes the chief difficulty of the problem, is usually effected by referring the body to a set of axes fixed in space, the position whereof is not arbitrary, but depends on the initial circumstances of the motion; viz. the axis of $z$ is taken to be perpendicular to the so-called invariable plane. The solution is obtained without this assumption both by Euler and Lagrange, although, as remarked by them, the formulæ are greatly simplified by making it; it is, on the other hand, made in the solution (which may be considered as the received one) by Poisson; and the results depending on it are the starting-point of the ulterior analytical developments of Rueb and Jacobi; the method of Poinsot is also based upon the consideration of the invariable plane. 
166. D'Alembert's Principle, which affords a direct and general method for obtaining the equations of motion in any dynamical problem whatever, was given in his "Traité de Dynamique" (1743); and in his memoir of 1749 he applied it to the physical problem of the Precession of the Equinoxes, which is a special case of the problem of Rotation, the motion of rotation about the centre of gravity being in fact similar to that about a fixed point. But, as might be expected in the first attempt at the analytical treatment of so difficult a problem, the equations of motion are obtained in a cumbrous and unmanageable form.

167. They are obtained by Euler in the memoir "Découverte d'un Nouveau Principe de Mécanique," Berlin Memoirs for 1750 (1752) (written before the establishment of the theory of principal axes), in a perfectly elegant form, including the ordinary one already mentioned, and, in fact, reducible to it by merely putting the quantities $F, G, H$ (which denote the integrals $\int y z d m$, \&c.) equal to zero. But Euler does not in this memoir enter into the question of the integration of the equations.

168. The notion of principal axes having been suggested by Euler, and their existence demonstrated by Segner, we come to Euler's investigations contained in the memoirs "Du Mouvement de Rotation \&c.," Berlin Memoirs for 1758 (printed 1765) and for 1760 (printed 1767), and the "Theoria Motus Corporum Solidorum \&c." (1765). In the memoir of 1760, and in the "Theoria Motus," Euler employs 8 , the angular velocity round the instantaneous axis, but not the resolved velocities $\gamma \cos \alpha, \gamma \cos \beta$, $\gamma \cos \gamma(=p, q, r)$ : these quantities (there called $x, y, z)$ are however employed in the memoir, Berlin Memoirs (1758), which must, I apprehend, have been written after the other, and in which at any rate the solution is developed with much greater completeness. It is in fact carried further than the ordinary solutions, and after the angular velocities $p, q, r$ have been found, the remaining investigation for the position in space of the principal axes, conducted, (as above remarked), without the aid of the invariable plane, is one of great elegance.

169. In the last-mentioned memoir Euler starts from the equations given by d'Alembert's principle; viz. the impressed forces being put equal to zero, these are

$$
\sum d m\left(y \frac{d^{2} z}{d t^{2}}-z \frac{d^{2} y}{d t^{2}}\right)=0, \quad \& c .
$$

or, what is the same thing, using $u, v, w$ to denote the velocities of an element in the directions of the axes fixed in space, these are

$$
\begin{aligned}
& \Sigma d m\left(y \frac{d w}{d t}-z \frac{d v}{d t}\right)=0 \\
& \Sigma d m\left(z \frac{d u}{d t}-x \frac{d w}{d t}\right)=0, \\
& \Sigma d m\left(x \frac{d v}{d t}-y \frac{d y}{d t}\right)=0 .
\end{aligned}
$$


It is assumed that at any moment the body revolves round an instantaneous axis, inclinations $\alpha, \beta, \gamma$, with an angular velocity $\gamma$; this gives

$$
\begin{aligned}
& u=8(z \cos \beta-y \cos \gamma)=q z-r y \\
& v=8(x \cos \gamma-z \cos \alpha)=r x-p z \\
& w=8(y \cos \alpha-x \cos \beta)=p x-q y
\end{aligned}
$$

if $\gamma \cos \alpha, \gamma \cos \beta, \gamma \cos \gamma$ are denoted by $p, q, r$. The values of $d u, d v$, dw are obtained by differentiating these formulæ, treating $p, q, r, x, y, z$ as variable, and replacing $d x, d y, d z$ by $u d t$, $v d t$, wdt respectively; in the resulting formulæ for $y d w-z d v$, \&c., $x, y, z$ are considered as denoting the coordinates of the element in regard to axes fixed in the body and moveable with it, but which at the moment under consideration coincide in position with the axes fixed in space. The expressions for $\Sigma(y d w-z d v) d m$ involve the integrals $A=\int\left(y^{2}+z^{2}\right) d m$, \&c., where the coordinates refer to axes fixed in the body; and if these are taken to be principal axes, the expression for $\Sigma(y d w-z d v) d m$ is $=A d p+(C-B) q r d t$, which gives the three equations

$$
\begin{aligned}
& A d p+(C-B) q r d t=0 \\
& B d q+(A-C) r p d t=0 \\
& C d r+(B-A) p q d t=0
\end{aligned}
$$

already referred to as Euler's equations.

170. Next, as regards the determination of the position in space of the principal axes: if about the fixed point we describe a sphere meeting the principal axes in $x_{1}, y_{1}, z_{1}$, and if $P$ be an arbitrary point on the sphere and $P Q$ an arbitrary direction through $P$, the quantities used to determine the positions of $x_{1}, y_{1}, z_{1}$ are the distances $x_{1} P, y_{1} P, z_{1} P(=l, m, n)$ and the inclinations $x_{1} P Q, y_{1} P Q, z_{1} P Q(=\lambda, \mu, \nu)$ of these arcs to the fixed direction $P Q$ (it is to be observed that the sines and cosines of the differences of $\lambda, \mu, \nu$ are given functions of the sines and cosines of $l, m, n$, and, moreover, that $\cos ^{2} l+\cos ^{2} m+\cos ^{2} n=1$, so that the number of independent parameters is three). The above is Euler's definition; but if we consider a set of axes fixed in space meeting the sphere in the points $X, Y, Z$, then if the point $X$ be taken for $P$, and the arc $X Y$ for $P Q$, it is at once seen that the angles used for determining the relative positions of the two sets of axes are the same as in Euler's memoir "Formulæ Generales \&c.", 1775 (ante, No. 132), where the formulæ for this transformation of coordinates are considered apart from the dynamical theory.

Euler expresses the quantities $p, q, r$ in terms of an auxiliary variable $u$, which is such that $d u=p q r d t ; p, q, r$ are at once obtained in terms of $u$, and then $t$ is given in terms of $u$ by a quadrature. Euler employs also an auxiliary angle $U$, given in terms of $u$ by a quadrature. And he obtains finite algebraical expressions in $u, \cos U, \sin U$ for the cosines or sines of $l, m, n ; s$ (the angular distance $I P$, if $I$ denote the point in which the instantaneous axis meets the sphere), $\phi$ (the angle $I P Q$ ) and $\lambda-\phi, \mu-\phi, \nu-\phi$. The formulæ, although complicated, are extremely elegant, and they appear to have been altogether overlooked by subsequent writers. 
171. Euler remarks, however, that the complexity of his solution is owing to the circumstance that the fixed point $P$ is left arbitrary, and that they may be simplified by taking this point so that a certain relation $G-D^{2}=0$ may be satisfied between the constants of the solution; and he gives the far more simple formulæ corresponding to this assumption. This amounts to taking the point $P$ in the normal of the invariable plane, and the resulting formulæ are in fact identical with the ordinary formulæ for the solution of the problem. The expression invariable plane is not used by Euler, and seems to have been first employed in Lagrange's memoir "Essai sur le Problème de Trois Corps," Prix de l'Acad. de Berlin, t. Ix. (1772): the theory in reference to the solar system has been studied by Laplace, Poinsot, and others.

172. Lagrange's solution in the memoir of 1773 is substantially the same with that in the Mécanique Analytique. Only he starts from the integral equations of areas and of Vis Viva, but in the last-mentioned work from the equations of motion as expressed in the Lagrangian form by means of the Vis Viva function $T\left(=\frac{1}{2} \Sigma\left(x^{\prime 2}+y^{\prime 2}+z^{2}\right) d m\right)$. The distinctive feature is that he does not refer the body to the principal axes but to any rectangular axes whatever fixed in the body: the expression for $T$ consequently is $T=\frac{1}{2}(A, B, C, F, G, H \gamma p, q, r)^{2}$, instead of the more simple form

$$
T=\frac{1}{2}\left(A p^{2}+B q^{2}+C r^{2}\right)
$$

which it assumes when the body is referred to its principal axes. And Lagrange effects the integration as well with this more general form of $T$, as without the simplification afforded by the invariable plane; the employment, however, of the more general form of $T$ seems an unnecessary complication of the problem, and the formulæ are not worked out nearly so completely as in Euler's memoir. It may be observed that $p, q, r$ are expressed as functions of the instantaneous velocity $\omega\left(=\sqrt{p^{2}+q^{2}+r^{2}}\right)$, and thence $t$ obtained by a quadrature as a function of $\omega$.

173. Poisson's Memoir of 1809.-The problem is only treated incidentally for the sake of obtaining the expressions for the variations of the arbitrary constants; the results (depending, as already remarked, on the consideration of the invariable plane) are obtained and exhibited in a very compact form, and they have served as a basis for further developments; it will be proper to refer to them somewhat particularly. The Eulerian equations give, in the first place, the integrals

$$
\begin{aligned}
& A p^{2}+B q^{2}+C r^{2}=h, \\
& A^{2} p^{2}+B^{2} q^{2}+C^{2} r^{2}=k^{2} ;
\end{aligned}
$$

and then by means of these, $p, q$ being expressed in terms of $r$, we have $t$ in terms of $r$ by a quadrature.

174: The position in space of the principal axes is determined by referring them, by means of the angles $\theta, \phi, \tau$, to axes $O x, O y, O z$ fixed in space; if, to fix the ideas, we call the plane of $x y$ the ecliptic ( $O x$ being the origin of longitudes), and the plane of the two principal axes $x_{1} y_{1}$ the equator, then we have

$\theta$, the longitude of node,

$\phi$, the inclination,

$\tau$, the hour-angle, or angular distance of $O x_{1}$ from the node,

C. IV. 
and $\alpha, \beta, \gamma$ the cosine inclinations of $O x_{1}, \alpha^{\prime}, \beta^{\prime}, \gamma^{\prime}$ those of $O y_{1}$, and $\alpha^{\prime \prime}, \beta^{\prime \prime}, \gamma^{\prime \prime}$ those of $O z_{1}$ to $O x, O y, O z$ respectively are given functions of $\theta, \phi, \tau$ (the values of $\alpha^{\prime \prime}, \beta^{\prime \prime}, \gamma^{\prime \prime}$ depending upon $\theta, \phi$ only), we have

$$
\begin{aligned}
& p d t=\sin \tau \sin \phi d \theta+\cos \tau d \phi \\
& q d t=\cos \tau \sin \phi d \theta-\sin \tau d \phi \\
& r d t=d \tau+\cos \phi d \theta
\end{aligned}
$$

175. A set of integrals is

$$
\begin{aligned}
& A p \alpha+B q \beta+C r \gamma=k \cos \lambda \\
& A p \alpha^{\prime}+B q \beta^{\prime}+C r \gamma^{\prime}=k \cos \mu \\
& A p \alpha^{\prime \prime}+B q \beta^{\prime \prime}+C r \gamma^{\prime \prime}=k \cos \nu
\end{aligned}
$$

equivalent to two independent equations, the values of $\lambda, \mu, \nu$ being such that $\cos ^{2} \lambda+\cos ^{2} \mu+\cos ^{2} \nu=1$; but the position of the axis of $z$ may be chosen so that the values on the right-hand sides become $0,0, k$; the axis of $z$ is then perpendicular to the invariable plane, the condition in question serving as a definition. And the three equations then give

$$
A p=k \alpha^{\prime \prime}, \quad B q=k \beta^{\prime \prime}, \quad C r=k \gamma^{\prime \prime},
$$

where the values of $\alpha^{\prime \prime}, \beta^{\prime \prime}, \gamma^{\prime \prime}$ in fact are

$$
\alpha^{\prime \prime}=\sin \tau \sin \phi, \quad \beta^{\prime \prime}=\cos \tau \sin \phi, \quad \gamma^{\prime \prime}=\cos \phi ;
$$

we have thus $\tau, \phi$ in terms of $r$. And the equation $r d t=d \tau+\cos \phi d \theta$ then leads to the value of $d \theta$, or $\theta$ is determined as a function of $r$ by a quadrature.

176. The constants of integration are $h, k, l$ (the constant attached to $t$ ), $g$ (the constant attached to $\theta$ ); and two constants, say $\alpha$ the longitude of the node, and $\gamma$ the inclination of the invariable plane in reference to an arbitrary plane of $x y$ and origin $x$ of longitudes therein. I remark in passing that Poisson obtains an elegant set of formulæ for the variations of the constants $h, k, g, l, \alpha, \gamma$, not actually in the canonical form, but which may by a slight change be reduced to it.

177. Legendre considers the problem of Rotation in the Exercices de Calcul Intégral, t. II. (1817), and the Théorie des Fonctions Elliptiques, t. I. pp. 366-410 (1826). He does not employ the quantities $p, q, r$, but obtains de novo a set of differential equations of the second order involving the angles which determine the position of the principal axes with regard to the axes fixed in space: these angles are in fact (calling the plane of the fixed axes $x, y$ the ecliptic) the longitude and latitude of one of the principal axes, and the azimuth from the meridian through such principal axis of an arbitrary axis fixed in the body and moveable with it. The solution is developed by means of the elliptic integrals. From the peculiar choice of variables there would, it would seem, be considerable labour in comparing the results with those of other writers, and there would be but little use in doing so. 
178. Poinsot's "Théorie Nouvelle de la Rotation des Corps."-The 'Extrait' of the memoir was published in 1834, but the memoir itself was not published in extenso. until the year 1851. The 'Extrait' contains, however, not only the fundamental theorem of the representation of the motion of a body about a fixed point by means of the momental ellipsoid rolling on a fixed tangent plane, but also the geometrical and mechanical reasonings by which this theorem is demonstrated; it establishes also the notions of the Poloid and Serpoloid curves; and it contains incidentally, and without any developments, a very important remark as to the representation of the motion by means of the rolling and sliding motion of an elliptic cone. The whole theory (including that of the last-mentioned representation of the motion) is in the memoir itself also analytically developed, but without the introduction of the elliptic and Jacobian functions: to form a complete theory, it would be necessary to incorporate the memoir with that of Jacobi.

179. The following is an outline of the 'Extrait':

The instantaneous motion of a body about a fixed point is a motion of rotation about an axis (the instantaneous axis); and hence the finite motion is as if there were a cone fixed in the body which rolls (without sliding) upon another cone fixed in space.

The instantaneous motion of a body in space is a motion of rotation about an axis combined with a translation in the direction of this axis: this remark is hardly required for Poinsot's purpose, and he does not further develope the theory of the motion of a body in space. The effect of a couple in a plane perpendicular to a principal axis is to turn the body about this axis with an angular velocity proportional to the moment of the couple divided by the moment of inertia about the axis.

And hence by resolving any couple into couples perpendicular to the principal axes, the effect of such couple may be calculated; but more simply by means of the central ellipsoid (momental ellipsoid $a^{2} x^{2}+b^{2} y^{2}+c^{2} z^{2}=k^{4}$, if $A, B, C=M a^{2}, M b^{2}, M c^{2}$ ), viz., if the body is acted on by a couple in a tangent plane of the ellipsoid, the instantaneous axis passes through the point of contact; and reciprocally, given the instantaneous axis, the couple must act in the tangent plane.

180. Considering now a body rotating about a fixed point, and taking as the plane of the couple of impulsion a tangent plane of the ellipsoid, the instantaneous axis is initially the diameter through the point of contact; the centrifugal forces arising from the rotation produce however an accelerating couple, the effect whereof is continually to impress on the body a rotation which is compounded with that about the instantaneous axis, and thus to cause a variation in the position of this axis and in the angular velocity round it. The axis of the accelerating couple is always situate in the plane of the couple of impulsion.

181. Hence also

$1^{\circ}$. Throughout the motion the angular velocity is proportional to the length of the instantaneous axis considered as a radius vector of the ellipsoid. 
$2^{\circ}$. The distance of the tangent plane from the centre is constant; that is, the tangent plane to the ellipsoid at the extremity of the instantaneous axis is a fixed plane in space.

Or, what is the same thing, the motion is such that the ellipsoid remains always in contact with a fixed plane, viz., the body revolves round the radius vector through the point of contact, the angular velocity being always proportional to the length of this radius vector.

It is right to remark that in Pcinsot's theory the distance of this plane from the centre depends on the arbitrarily assumed magnitude of the central ellipsoid; the parallel plane through the centre is the invariable plane of the motion.

182. The motion is best understood by the consideration that it is implied in the theorem that the pole of the instantaneous axis describes on the ellipsoid a certain curve, "the Poloid," which is the locus of all the points for which the perpendicular on the tangent plane has a given constant value (the curve in question is easily seen to be the intersection of the ellipsoid by a concentric cone of the second order); and that the instantaneous axis describes on the fixed tangent plane a curve called "the Serpoloid," which is the locus of the points with which the several points of the poloid come successively in contact with the tangent plane, and is a species of undulating curve, viz., the radius vector as it moves through the angles $\theta$ to $\theta_{1}+2 \Pi, \theta_{1}+2 \Pi$ to $\theta_{1}+4 \Pi$, \&c. assumes continually the same series of values. This is in fact evident from the mode of generation; and it is moreover clear that the serpoloid is an algebraical or else a transcendental curve according as $\Pi$ is or is not commensurable with $\pi$.

\{Treating the poloid and serpoloid as cones instead of curves, the motion of the body is the rolling motion of the former upon the latter cone, which agrees with a previous remark.\}

There is a very interesting special case where the perpendicular distance from the tangent plane is equal to the mean axis of the ellipse.

183. Poinsot remarks that the motion is such that \{considering the plane of the couple of impulsion as drawn through the centre of the ellipsoid\} the section of the ellipsoid is an ellipse variable in form but of constant magnitude, and that this leads to a new representation of the motion, viz., that it may be regarded as the motion of an elliptic cone which rolls on the plane of the couple \{the invariable plane\} with a variable velocity, and which slides on this plane with a uniform velocity.

184. The theory of the last-mentioned cone, say the "rolling and sliding cone," is developed in the memoir, Liouville, t. XVI. p. 303, in the chapter entitled "Nouvelle Image de la Rotation des Corps." If $a, b, c$ signify as before (viz., $A, B, C=M a^{2}, M b^{2}, M c^{2}$ ), and if $h$ be the distance of the centre from Poinsot's fixed tangent plane $(h<a>c)$, then the invariable axis describes in the body a cone the equation whereof is

$$
\left(a^{2}-h^{2}\right) x^{2}+\left(b^{2}-h^{2}\right) y^{2}+\left(c^{2}-h^{2}\right) z^{2}=0 ;
$$

the cone reciprocal to this, viz. the cone the equation whereof is

$$
\frac{x^{2}}{a^{2}-h^{2}}+\frac{y^{2}}{b^{2}-h^{2}}+\frac{z^{2}}{c^{2}-h^{2}}=0,
$$


is the "rolling and sliding cone." The generating line $O T$ of this cone is perpendicular to the plane of the instantaneous axis $O I$, and of the invariable axis $O G$; and the analytical expressions for the rolling and sliding velocities follow from the geometrical consideration that the motion at any instant is a rotation round the instantaneous axis $O I$ : that for the sliding velocity is the instantaneous angular velocity into the cosine of the angle IOG, which is in fact constant; that for the rolling velocity is given, but a further explanation of the geometrical signification is perhaps desirable.

185. I may in this place again refer to Cohen's memoir "On the Differential Coefficients and Determinants of Lines \&c." (1862), the latter part of which contains an application of the method to finding Euler's equations for the motion of a rotating body.

186. Rueb in his memoir (1834) first applied the elliptic and Jacobian functions to the present problem. Starting from the equations

and

$$
\begin{aligned}
& A p^{2}+B q^{2}+C r^{2}=h, \\
& \left.A^{2} p^{2}+B^{2} q^{2}+C^{2} r^{2}=l^{2},{ }^{1}\right)
\end{aligned}
$$

$$
d t=\frac{-B d q}{(A-C) r p},
$$

it is easy to perceive that by assuming $q=a$ proper multiple of $\sin \xi$, the expression for $d t$ takes the form $n d t=\frac{d \xi}{\sqrt{1-k^{2} \sin ^{2} \xi}}$, so that writing $\xi=\operatorname{am} u$, the integral equation is $n t-\epsilon=u$, or $u$ is an angle varying directly as the time (and corresponding to the mean longitude, or, if we please, to the mean anomaly in the problem of elliptic motion). And then $p, q, r$ are expressed as elliptic functions of $u$. The value of the modulus $k$, and that of $n(n t-\epsilon=u$ ut suprà) are

$$
\begin{aligned}
& n=\sqrt{\frac{(B-C)\left(-l^{2}+A h\right)}{A B C},} \\
& k=\sqrt{\frac{(A-B)\left(l^{2}-C h\right)}{A B C}},
\end{aligned}
$$

and then

$$
\left.\begin{array}{l}
p= \pm \sqrt{\frac{l^{2}-C h}{A \cdot A-C}} \cos \text { am } u, \\
q=-\sqrt{\frac{l^{2}-C h}{B \cdot B-C}} \sin \text { am } u, \\
r=\sqrt{\frac{-l^{2}+A h}{C \cdot A-C}} \Delta \text { am } u .
\end{array}\right\}
$$

$1 l$ is Poisson's $k$, the constant of the principal area; it is the letter afterwards used by Jacobi ; Rueb's letter is $g$. In quoting (infrà) the expressions for $p, q, r$, I have given them with Rueb's signs, but it would be too long to explain how the signs of the radicals are determined. 
187. Substituting for $p, q, r$ their values in terms of $u$, we have $d \theta$, and thence $\theta$ (the longitude of the node of the equator on the invariable plane) in the form

$$
\theta=-\frac{l}{A n} u+i \Pi(u, i a) \quad(i=\sqrt{-1})
$$

which by Jacobi's formulæ for the transformation of the elliptic integral of the third class becomes

$$
\theta=\left(-\frac{l}{A n}+i Z(a i)\right) u+\frac{1}{2} i \log \frac{\Theta(u-a i)}{\Theta(u+a i)},
$$

which Rueb reduces to the real form

$$
\theta=-n^{\prime} u+\tan ^{-1} W,
$$

$W$ being given in the form of a fraction, the numerator and denominator whereof are series in multiple sines and multiple cosines respectively of $\frac{\pi u}{2 K}$.

188. Rueb investigates also the values in terms of $u$ of the cosine inclinations of the instantaneous axis to the axes fixed in space; and he obtains a very elegant expression for the angle $\zeta$, which is the angular distance from $x$ of the projection on the plane of $x y$ (the invariable plane) of the instantaneous axis; viz., this is

$$
\zeta=\tan ^{-1}\left(-\frac{A B n}{(A-B) l} \frac{\Delta \operatorname{am} u}{\sin \operatorname{am} u \cos \operatorname{am} u}\right)-\theta,
$$

and there is throughout a careful discussion of the geometrical signification of the results.

189. The advance made was enormous; the result is that we have in terms of the time $\sin \tau \sin \phi, \cos \tau \sin \phi, \cos \phi$ (the cosine inclinations of the invariable axis to the principal axes), and also $\theta$, the longitude of the node. The cosine inclinations of the axes of $x$ and $y$ to the principal axes could of course be obtained from these, but they would be of a very complicated and unmanageable form; the reason of this is the presence in the expression for $\theta$ of the non-periodic term - n'u. It will presently be seen how this difficulty was got over by Jacobi.

190. Briot's paper of 1842 contains an analytical demonstration of some of the theorems given in the 'Extrait' of Poinsot's memoir of 1834 .

191. In Maccullagh's Lectures of 1844 (see Haughton, 1849; Maccullagh, 1847) the problem of the rotation of a solid body is treated in a mode somewhat similar to that of Poinsot; only the ellipsoid of gyration $\left(\frac{x^{2}}{a^{2}}+\frac{y^{2}}{b^{2}}+\frac{z^{2}}{c^{2}}=1\right.$, if $\left.A, B, C=M a^{2}, M b^{2}, M c^{2}\right)$ is used instead of the momental ellipsoid. Thus, reciprocal to the poloid curve on the momental ellipsoid we have on the ellipsoid of gyration a curve all the points whereof are equidistant from the centre; such curve is of course the intersection of the ellipsoid by a concentric sphere, that is, it is a spherical conic; and the points of this spherical conic come successively to coincide with a fixed point on the invariable axis. This is a theorem stated in Art. VI. of Haughton's memoir: it may be added 
that the several tangent planes of the ellipsoid of gyration at the points of the spherical conic as they come to coincide with the fixed point, form a cone reciprocal to Poinsot's serpoloid cone. It is clear that every theorem in the one theory has its reciprocal in the other theory; I have not particularly examined as to how far the reciprocal theorems have been stated in the two theories.

192. Cayley, "On the Motion of Rotation of a Solid Body" (1843).-The object was to apply to the solution of the problem Rodrigues' formulæ for the resultant rotation; viz., if the principal axes, considered as originally coinciding with the axes of $x, y, z$, can be brought into their actual position at the end of the time $t$ by a rotation $\theta$ round an axis inclined at angles $f, g, h$ to the axes of $x, y, z$, and if $\lambda=\tan \frac{1}{2} \theta \cos f, \mu=\tan \frac{1}{2} \theta \cos g$, $\nu=\tan \frac{1}{2} \theta \cos h$, then the principal axes are referred to the axes fixed in space by means of the quantities $\lambda, \mu, \nu$. And these are to be obtained from the equations

$$
\begin{aligned}
& \kappa p d t=2(\quad d \lambda+\nu d \mu-\mu d \nu), \\
& \kappa q d t=2(-\nu d \lambda+d \mu+\lambda d \nu), \\
& \kappa r d t=2(\quad \mu d \lambda-\lambda d \mu+d \nu),
\end{aligned}
$$

where $\kappa=1+\lambda^{2}+\mu^{2}+\nu^{2}$, and $p, q, r$ are to be considered as given functions of $t$, or of other the variable selected as the independent one. But for effecting the integration it was found necessary to take the axis of $z$ as the invariable axis.

193. The solution by Jacobi, $\S 27$ of the memoir "Theoria Novi Multiplicatoris" (1845), is given as an application of the general theory, the author remarking that, as well in this question as in the problem of the two fixed centres, he purposely employed a somewhat inartificial analysis, in order to show that the principle (of the Ultimate Multiplier) would lead to the result without any special artifices. The principal axes are referred to the axes fixed in space by the ordinary three angles (here called $q_{1}, q_{2}, q_{3}$ ), and the solution is carried so far as to give the integral equations, without any reduction of the integrals contained in them to elliptic integrals. The solution is, however, in so far remarkable that the integrations are effected without the aid of the invariable plane.

194. Cayley, "On the Rotation of a Solid Body \&c." (1846).-It appeared desirable to obtain the solution by means of the quantities $\lambda, \mu, \nu$, without the assistance of the invariable plane, and Jacobi's discovery of the theorem of the Ultimate Multiplier induced me to resume the problem, and at least attempt to bring it so far as to obtain a differential equation of the first order between two variables only, the multiplier of which could be obtained theoretically by Jacobi's discovery. The choice of two new variables to which the equations of the problem led me, enabled me to effect this in a simple manner; and the differential equation which I finally obtained turned out to be integrable per se, so that the laborious process of finding the multiplier became unnecessary.

195. The new variables $\Omega, v$ have the following geometrical significations: $\Omega=l \tan \frac{1}{2} \theta \cos I$, where $l$ is the principal moment $\left(A^{2} p^{2}+B^{2} q^{2}+C^{2} r^{2}=l^{2}\right), \theta$ (as before) the angle of resultant 
rotation, and $I$ is the inclination of the resultant axis to the invariable axis; and $v=l^{2} \cos ^{2} \frac{1}{2} J$, where if we imagine a line $A Q$ having the same position relatively to the axes fixed in space that the invariable axis has to the principal axes of the body, then $J$ is the inclination of this line to the invariable axis. It is found that $p, q, r$ are functions of $v$ only, whereas $\lambda, \mu, \nu$ contain besides the variable $\Omega$. In obtaining these relations, there occurs a singular relation $\Omega^{2}=\kappa v-l^{2}$, which may also be written $1+\tan ^{2} \frac{1}{2} \theta \cos ^{2} I=\sec ^{2} \frac{1}{2} \theta \cos ^{2} \frac{1}{2} J$, where the geometrical significations of the quantities $I, J$ have just been explained. The final results are that the time $t$, and the arc $\tan ^{-1} \frac{\Omega}{l}$ are each of them expressible as the integrals of certain algebraical functions of $v$. There might be some interest in comparing the results with those of Euler's memoir of 1758 , where the principal axes are also referred to an arbitrary system of axes fixed in space; but I was not then acquainted with Euler's memoir.

The concluding part of the paper relates to the determination of the variations of the constants in the disturbed problem.

196. Cayley, "Note on the Rotation of a Solid of Revolution" (1849), shows the simplification produced in the formulæ of the last-mentioned memoir in the case where two of the moments of inertia are equal, say $A=B$.

197. Jacobi's final solution of the problem of Rotation was given without demonstration in the letter to the Academy of Sciences at Paris; the demonstration is added in the memoir, Crelle, t. xxxIx. (1849). The fundamental idea consists in taking in the invariable plane, instead of the fixed axes $x y$, a set of axes $x y$ revolving with uniform velocity, such that the angular distance of the axis of $x$ from its initial position is precisely $=-n^{\prime} u$; and consequently if $\theta^{\prime}$ be the longitude of the node of the equator on the invariable plane, measured from the moveable axis of $x$ as the origin of longitude, we have

$$
\theta^{\prime}=\theta+n^{\prime} u=\frac{1}{2 i} \log \frac{\Theta(u+i a)}{\Theta(u-i a)}, \quad(i=\sqrt{-1}) ;
$$

and in consequence of this form of the expression for $\theta^{\prime}\left(=\frac{1}{2 i}\right.$ into a logarithmic function $)$ in passing to the trigonometrical functions $\sin \theta^{\prime}, \cos \theta^{\prime}$ the logarithm disappears altogether; and we have in a simple form the expressions for the actual functions $\sin \theta^{\prime}, \cos \theta^{\prime}$, through which $\theta^{\prime}$ enters into the formulæ, and thus, Jacobi remarks, the barrier is cleared which stands in the way when the expression of an angle is reduced to an elliptic integral of the third class.

198. For the better expression of the results, Jacobi joins to the functions $H, \Theta$, considered in the "Fundamenta Nova," the functions $\Theta_{1} u=\Theta(K-u), H_{1}(u)=H(K-u)$; so that

$$
\sqrt{k} \sin \operatorname{am} u=\frac{H u}{\Theta u}, \quad \sqrt{\frac{k}{k^{\prime}}} \cos \operatorname{am} u=\frac{H_{1} u}{\Theta u}, \quad \frac{1}{\sqrt{k^{\prime}}} \Delta \operatorname{am} u=\frac{\Theta_{1} u}{\Theta u},
$$

and then considering the cosine inclinations of the principal axes to the invariable axis and the revolving axes in the invariable plane, these are all fractions which, 
neglecting constant factors, have the common denominator $\Theta u ; \alpha^{\prime \prime}, \beta^{\prime \prime}, \gamma^{\prime \prime}$ (as shown by Rueb's formulæ) have the numerators $H_{1} u, H u$, and $\Theta_{1} u$ respectively; and $\alpha, \alpha^{\prime}$ have the numerators $H(u+i a) \pm H(u-i a), \beta, \beta^{\prime}$ the numerators $H_{1}(u-i a) \pm H_{1}(u+i a), \gamma, \gamma^{\prime}$ the numerators $\Theta(u+i a) \pm \Theta(u-i a)$ respectively: there are also expressions of a similar form for the angular velocities about the axis of $x$ and $y$; that about the axis of $z$ (the invariable axis) having, as was known, the constant value $\frac{h}{l}$. The memoir is also very valuable analytically, as completing the systems of formulæ given in the Fundamenta Nova in reference to elliptic integrals of the thịd class.

199. It is worth noticing how the results connect themselves with Poinsot's theorem of the rolling and sliding cone: the velocity of the rolling motion depends only upon the position, on the cone, of the line of contact, so that the same series of velocities recur after any number of complete revolutions of the cone; that is, the total angle described by the line of contact in consequence of the rolling motion, consists of a part varying directly with the time (or say varying as $u$ ) and a periodic part; the former part combines with the similar term arising from the sliding motion, and the two together give Jacobi's term $n^{\prime} u$.

200. Somoff's memoir (1851), written after Jacobi's Note in the Comptes Rendus, but before the appearance of the memoir in Crelle, gives the demonstration of the greater part of Jacobi's results.

201. Booth's Theory of Elliptic Integrals \&c. (1851) (contemporaneous with the publication of Poinsot's memoir of 1834) contains various interesting analytical developments, and, as an interpretation of them, the author obtains (p. 93) the theorem of the rolling and sliding cone. The investigations involve the elliptic integrals, but not the elliptic or Jacobian functions.

202. Richelot's two Notes (Crelle, tt. XLII. and XLIV.) relate to the solution of the problem of rotation given in his memoir "Eine neue Lösung \&c." (1851). This is an application of Jacobi's theorem for the integration of a system of dynamical equations by means of the principal function $S$ (see my "Report" of 1857, art. 34). Retaining Richelot's letters $\phi, \psi, \theta$, which signify

$$
\begin{aligned}
& \psi \text {, the longitude of the node, } \\
& \theta \text {, the inclination, } \\
& \phi \text {, the hour-angle, }
\end{aligned}
$$

the question is to find a complete solution of the partial differential equation

$$
\begin{aligned}
0= & \frac{1}{2 A}\left\{\left(\frac{d V}{d \phi} \cos \theta+\frac{d V}{d \psi}\right)^{2} \sin \phi\right. \\
\sin \theta & \left.-\frac{d V}{d \theta} \cos \phi\right\}^{2} \\
& +\frac{1}{2 B}\left\{\left(\frac{d V}{d \phi} \cos \theta+\frac{d V}{d \psi}\right) \frac{\cos \phi}{\sin \theta}+\frac{d V}{d \theta} \sin \phi\right\}^{2} \\
& +\frac{1}{2 C}\left(\frac{d V}{d \phi}\right)^{2}+\frac{d V}{d t}
\end{aligned}
$$

C. IV. 
that is, a solution involving (besides the constant attached to $V$ by a mere addition) three arbitrary constants; these are $t_{1}, \psi_{1}, \rho$. Writing in the first place $V=W+t t_{1}+\psi \psi_{1}$, the resulting equation for $W$ may be satisfied by taking $W$, a function of $\phi$ and $\theta$, without $\psi$ or $t$; and it is sufficient to have a solution involving only a single arbitrary constant. This leads to a solution which is as follows:

$$
\begin{aligned}
V=t t_{1}+\psi \psi_{1}-\psi_{1} & \tan ^{-1} \frac{\theta_{1}}{\sqrt{\rho^{2}-\psi_{1}{ }^{2}-\theta_{1}{ }^{2}}}+\rho\left\{\tan ^{-1} \frac{\psi_{1} \theta_{1}}{\rho \sqrt{\rho^{2}-\psi_{1}{ }^{2}-\theta_{1}{ }^{2}}}+\tan ^{-1} \frac{\phi_{1} \theta_{1}}{\rho \sqrt{\rho^{2}-\phi_{1}{ }^{2}-\theta_{1}}}\right\} \\
& -\left(\frac{\rho^{2}}{C}+2 t_{1}\right) \int \frac{\phi_{1}{ }^{2} d \phi_{1}}{\left(\rho^{2}-\phi_{1}{ }^{2}\right) \sqrt{\left(\frac{1}{B}-\frac{1}{C}\right)\left(\phi_{1}{ }^{2}-\frac{\rho^{2}}{B}+2 t_{1}\right)\left(\left(\frac{1}{C}-\frac{1}{A}\right) \phi_{1}{ }^{2}+\frac{\rho^{2}}{A}+2 t_{1}\right)}}
\end{aligned}
$$

where $\phi_{1}$ and $\theta_{1}$ are certain given functions of $t_{1}, \psi_{1}, \rho$, and of $\theta$ and $\phi$. The solution of the dynamical problem is then obtained by putting the differential coefficients $\frac{d V}{d t_{1}}, \frac{d V}{d \psi_{1}}, \frac{d V}{d \rho}$ equal to arbitrary constants $L, \alpha, G$ respectively; the results are somewhat more simple than might be expected from the very complicated form of the function $V$. The foregoing results (although not by themselves very intelligible) will give an idea of the form in which the analytical solution in the first instance presents itself.

203. Richelot proceeds to remark that the solution in question, and the resulting integral equations of the problem, may be simplified in a peculiar manner by the method which he calls "the integration by the spherical triangle." For this purpose he introduces a spherical triangle, the sides and angles whereof are

and then assuming

$$
\nu, \lambda, \mu ; \mathrm{N}, \Lambda, \mathrm{M} \text {, }
$$

$$
\mathrm{N} \text { constant, } \mathrm{M}=\pi=\theta
$$

$$
\left(\frac{1}{C}-\frac{1}{A}\right) \sin ^{2}(\phi+\nu) \sin ^{2} \Lambda+\left(\frac{1}{C}-\frac{1}{B}\right) \cos ^{2}(\phi+\nu) \sin ^{2} \Lambda=\frac{1}{C}+\frac{2 t_{1}}{\rho^{2}},
$$

where $\rho$ and $t_{1}$ are constant, the solution is

$$
V=t_{1} t-\rho(\psi-\lambda) \cos \mathbf{N}-\rho \mathbf{M}+\rho \int \cos \Lambda d(\phi+\nu) ;
$$

and that this expression leads to all the results almost without calculation.

204. I have quoted the foregoing results from the Note (Crelle, t. xLII.), having seen, but without having studied, the Memoir itself: the results appear very interesting and valuable ones; but they seem to require a more complete geometrical development than they have received in the Memoir; and I am not able to bring them into connexion with the other researches on the subject.

205. The solution, $\S 3$ of Donkin's memoir "On a Class of Differential Equations \&c." (part I. 1854), is given as an illustration of the general theory to which the memoir relates; it contains, however, some interesting geometrical developments in regard to the case $(A=B)$ of two equal moments of inertia. I have not compared the results with those in my Note of 1849 . 
206. The solution of the rotation problem, $\S 66$ of Jacobi's memoir "Nova Methodus \&c." (1862), has for its object to show the complete analogy which exists between this problem and the problem of a body attracted to a fixed centre. The section is in fact headed "Solutio simultanea problematis de motu puncti versus centrum attracti atque problematis de rotatione \&c."; and Jacobi, after noticing that Poisson, in his memoir of 1816 (Mém. de l'Inst. t. I.), had shown that the expressions for the variations of the elements in the two problems could be investigated by a common analysis, remarks, "Sed ipsa problemata duo imperturbata hic primum, quantum credo, amplexus sum." The solution is in fact as follows:-Suppose that in the one problem the position of the point in space, and in the other problem the position of the body in regard to the fixed axes, is determined in any manner by the quantities $q_{1}, q_{2}, q_{3}$. Let

$$
\frac{d q_{1}}{d t}=q_{1}^{\prime}, \quad \frac{d q_{2}}{d t}=q_{2}^{\prime}, \quad \frac{d q_{3}}{d t}=q_{3}^{\prime},
$$

and expressing the Vis Viva function $T$ in terms of $q_{1}, q_{2}, q_{3}, q_{1}^{\prime}, q_{2}^{\prime}, q_{3}^{\prime}$, let

$$
\frac{d T}{d q_{1}^{\prime}}=p_{1}, \quad \frac{d T}{d q_{2}^{\prime}}=p_{2}, \quad \frac{d T}{d q_{3}^{\prime}}=p_{3},
$$

and let $H$ be the value of $T$ expressed in terms of $q_{1}, q_{2}, q_{3}, p_{1}, p_{2}, p_{3}$, so that $H=a$ is the integral of Vis Viva (this is merely the transformation to the Hamiltonian form). And let $H_{1}=a_{1}, \phi=a_{1}^{\prime}, \psi=a_{1}^{\prime \prime}$ be the three integrals of areas $\left(H, H_{1}, \phi, \psi\right.$ are functions of the variables only, not containing the arbitrary constants $\left.a, a_{1}, a_{1}^{\prime}, a_{1}^{\prime \prime}\right)$. Then, expressing

$$
H, H_{1}, H_{2}\left(=\sqrt{H_{1}^{2}+\phi^{2}+\psi^{2}}\right)
$$

in terms of $p_{1}, p_{2}, p_{3}, q_{1}, q_{2}, q_{3}$, and by means of the equations

$$
H=a, \quad H_{1}=a_{1}, \quad H_{2}=a_{2}
$$

(where $a_{2}=\sqrt{a_{1}^{2}+a_{1}^{\prime 2}+a_{1}^{\prime \prime 2}}$ ) expressing $p_{1}, p_{2}, p_{3}$ in terms of $q_{1}, q_{2}, q_{3}$, we have $p_{1} d q_{1}+p_{2} d q_{2}+p_{3} d q_{3}$ a complete differential ; and putting

$$
\int\left(p_{1} d q_{1}+p_{2} d q_{2}+p_{3} d q_{3}\right)=V
$$

then ( $a, a_{1}, a_{2}, b, b_{1}, b_{2}$ being arbitrary constants) we have

$$
\begin{gathered}
H=a, \quad H_{1}=a_{1}, \quad H_{2}=a_{2}, \\
\frac{d V}{d a}=\int\left(\frac{d p_{1}}{d a} d q_{1}+\frac{d p_{2}}{d a} d q_{2}+\frac{d p_{3}}{d a} d q_{3}\right)=t+b, \\
\frac{d V}{d a_{1}}=\int\left(\frac{d p_{1}}{d a_{1}} d q_{1}+\frac{d p_{2}}{d a_{1}} d q_{2}+\frac{d p_{3}}{d a_{1}} d q_{3}\right)=b_{1}, \\
\frac{d V}{d a_{2}}=\int\left(\frac{d p_{1}}{d a_{2}} d q_{1}+\frac{d p_{2}}{d a_{2}} d q_{2}+\frac{d p_{3}}{d a_{2}} d q_{3}\right)=b_{2},
\end{gathered}
$$


as the complete integrals of either problem, the last three of them being the final integrals.

And it is added that if in either problem we have $H+\Omega$ instead of $H$, the expressions for the variations of the elements assume the canonical forms $\frac{d a}{d t}=-\frac{d \Omega}{d b}, \frac{d b}{d t}=\frac{d \Omega}{d a}$, \&c.

The solution is not further developed as regards the rotation problem, but it is so $(\S 67)$ as regards the other problem.

207. It must, I think, be considered that a comprehensive memoir on the Problem of Rotation, embracing and incorporating all that has been done on the subject, is greatly needed.

\section{Kinematics of a solid body. Article Nos. 208 to 215.}

208. The general theorem in regard to the infinitesimal motions (rotations and translations) of a solid body is that these are compounded and resolved in the same way as if they were single forces and couples respectively. Thus any infinitesimal rotations and translations are resolvable into a rotation and a translation; the rotation is given as to its magnitude and as to the direction of its axis, but not as to the position of the axis (which may be any line in the given direction): the magnitude and direction of the translation depend on the assumed position of the axis of rotation; in particular this may be taken so that the translation shall be in the direction of the axis of rotation; and the magnitude of the rotation is then a minimum. I remark that the theorem as above stated presupposes the establishment of the theory of couples (of forces) which was first accomplished by Poinsot in his 'Élémens de Statique,' 1st edit. 1804; it must have been, the whole or nearly the whole of it, familiar to Chasles at the date of his paper of 1830 next referred to (see-also Note XXXIV. of the Aperçu Historique, 1837); and it is nearly the whole of it stated in the 'Extrait' of Poinsot's memoir on Rotation, 1834.

209. Chasles' paper in the Bulletin Univ. des Sciences for 1830.-The corresponding theorem is here given for the finite motions (rotations and translations) of a solid body as follows: viz. if any finite displacement be given to a free solid body in space, there exists always in the body a certain indefinite line which after the displacement remains in its original situation. The theorem is deduced from a more general one relating to two similar bodies. It may be otherwise stated thus: viz., any motions may be represented by a translation and a rotation (the order of the two being indifferent); the rotation is given as regards its magnitude and the direction of its axis, but not as to the position of the axis (which may be any line in the given direction); the magnitude and direction of the translation depend on the assumed position of the axis of rotation; in particular this may be taken so that the translation shall be in the direction of the axis of rotation; the magnitude of the translation is then a minimum.

It may be noticed that a translation may be represented as a couple of rotations; that is, two equal and opposite rotations about parallel axes. 
210. It is part of the general theorem that any number of rotations about axes passing through one and the same point may be compounded into a single rotation about an axis through that point; this is, in fact, the theory of the "Resultant Axis" developed in Euler's and Lexell's memoirs of 1775.

211. The following properties are also given, viz., considering two similar solid bodies (or in particular any two positions of a solid body) and joining the corresponding points, the lines which pass through one and the same point form a cone of the second order; and the points of either body form on this cone a curve of the third order (skew cubic). And, reciprocally, the lines, intersections of corresponding planes, which lie in one and the same plane envelope a conic, and such planes of either body envelope a developable surface, which is such that any one of these planes meets it in a conic \{or, what is the same thing, the planes envelope a developable surface of the fourth order\}.

And also, given in space two equal bodies situate in any manner in respect to each other, then joining the points of the first body to the homologous points of the second body, the middle points of these lines form a body capable of an infinitesimal motion, each point of it along the line on which the same is situate.

212. The entire theory, as well of the infinitesimal as of the finite motions of a solid body, is carefully and successfully treated in Rodrigues' memoir "Des lois géométriques \&c." (1840). It may be remarked that for the purpose of compounding together any rotations and translations, each rotation may be resolved into a rotation about a parallel axis and a couple of rotations, that is, a translation; the rotations are thus converted into rotations about axes through one and the same point, and these give rise to a single resultant rotation given as to its magnitude and the direction of the axis, but not as to the position of the axis (which is an arbitrary line in the given direction); the translations are then compounded together into a single translation, and finally the position of the axis of rotation is so determined that the translation shall be in the direction of this axis; the entire system is thus compounded (in accordance with Chasles' theorem) into a rotation and a translation in the direction of the axis of the rotation. The problem of the composition depends therefore on the composition of rotations about axes through one and the same point; that is, upon Euler's and Lexell's theory of the resultant axis. But, as already noticed, the analytical theory of the resultant axis was perfected by Rodrigues in the present memoir (see ante, 'Transformation of Coordinates,' Nos. 139-141, as to this memoir and the quaternion representation of the formulæ contained in it.

213. It was remarked in Poinsot's memoir of 1834 that every continuous motion of a solid body about a fixed point is the motion of a cone fixed in the body rolling upon another cone fixed in space. The corresponding theorem for the motion of a solid body in space is given

Cayley, "On the Geometrical Representation \&c." (1846): viz. premising that a skew surface is said to be "deformed" if, considering the elements between consecutive generating lines as rigid, these elements be made in any manner to turn round and 
slide along the successive generating lines:-and that two skew surfaces can be made to roll and slide one upon the other, only if the one is a deformation of the otherand that then the rolling and sliding motions are perfectly determined-and that such a motion may be said to be a "gliding" motion: the theorem is that any motion whatever of a solid body in space may be represented as the gliding motion of one skew surface upon another skew surface of which it is the deformation.

214. The same paper contains the enunciation and analytical proof of the following theorem supplementary to that of Poinsot just referred to, viz. that when the motion of a solid body round a fixed point is represented as the rolling motion of one cone on another, then "the angular velocity round the line of contact (the instantaneous axis) is to the angular velocity of this line as the difference of the curvatures of the two cones at any point in this line is to the reciprocal of the distance of the point from the vertex."

215. There are a great number of theorems relating to the composition of forces and force-couples, which consequently relate also to infinitesimal rotations and translations. See, for instance, Chasles, "Théorèmes généraux \&c." (1847), Möbius, "Lehrbuch der Statik" (1837), Steichen's Memoirs of 1853 and 1854, \&c. Arising out of some theorems of Möbius in the "Statik," we have Sylvester's theory of the involution of six lines: viz. six lines (given in position) may be such that properly selected forces along them (or if we please, properly selected infinitesimal rotations round them) will counterbalance each other; or, what is the same thing, the six lines may be such that a system of forces, although satisfying for each of the six lines the condition moment $=0$, will not of necessity be in equilibrium; such six lines are said to be in involution, and the geometrical theory is a very extensive and interesting one.

\section{Miscellaneous Problems. Article Nos. 216 to 223.}

216. As under the foregoing head, "Rotation round a fixed point," I have considered only the case of a body not acted upon by any forces, the case where the body is acted upon by any forces comes under the present head. The forces, whatever they are, may be considered as disturbing forces, and the problem be treated by the method of the variation of the elements; this is at any rate a separate part of the theory of rotation round a fixed point, and I have found it convenient to include it under the present head; the only case in which the forces have been treated as principal ones, seems to be that of a heavy body (a solid of revolution) rotating about a point not its centre of gravity. The case of a body suspended by a thread or resting on a plane comes under the present head, as also would (in some at least of the questions connected with it) the gyroscope. But none of these questions are here considered in any detail.

\section{Rotation round a fixed point-Variation of the elements.}

217. The forces acting on the body are treated as disturbing forces. Formulæ for the variations of the elements were first obtained by Poisson in the memoir "Sur la Variation des Constantes Arbitraires \&c." (1809). The variations are expressed in terms 
of the differential coefficients of the disturbing function in regard to the elements, and, as the author remarks, they are very similar in their form to, and can be rendered identical with, those for the variations of the elements in the theory of elliptic motion.

218. Cayley, "On the Rotation \&c." (1846).-The latter part of the paper relates to the variations of the elements therein made use of, which are different from the ordinary ones.

219. Richelot, "Eine neue Lösung \&c." (1851).-The form in which the integrals are obtained by means of a function $V$, satisfying a partial differential equation, leads at once to a canonical system for the variations of the elements; these formulæ are referred to in the introduction to the memoir, but they are not afterwards considered.

220. Cayley, "On the Rotation of a Solid Body" (1860).-The elements are those ordinarily made use of, with only a slight variation occasioned by the employment of the "departure" of the node. The course of the investigation consists in obtaining the variations in terms of the differential coefficients of the disturbing function in regard to the coordinates (formulæ which were thought interesting for their own sake), and in deducing therefrom those in terms of the differential coefficients in terms of the elements.

\section{Other cases of the motion of a solid body.}

221. In regard to a heavy solid of revolution rotating about a fixed point not its centre of gravity, we have

Poisson, "Mémoire sur un cas particulier \&c." (1831), and the elaborate memoir

Lottner, "Reduction der Bewegung \&c." (1855), where the solution is worked out by means of the Elliptic and Jacobian functions.

222. As regards a heavy solid suspended by a string,

Pagani, "Mémoire sur l'équilibre \&c." (1839).

223. As regards the motion of a body resting on a fixed plane,

Cournot, "Mémoire sur le Mouvement \&c." (1830 and 1832).

Puiseux, "Du Mouvement \&c." (1848).

To these several others might doubtless be added; but the problems are so difficult, that the solutions cannot, it is probable, be obtained in any very complete form.

In conclusion, I can only regret that, notwithstanding the time which has elapsed since the present Report was undertaken, it is still-both as regards the omission of memoirs and works which should have been noticed, and the merely cursory examination of some of those which are mentioned-far from being as complete as I should have wished to make it. To have reproduced, to any much greater extent than has been 
done, the various mathematical investigations, would not have been proper, nor indeed practicable; at the same time, more especially as regards the subjects treated of in the second part of this Report, or say the kinematics and dynamics of a solid body, such a reproduction, incorporating and to some extent harmonizing the original researches, but without ignoring the points of view and methods of investigation of the several authors, would be a work which would well repay the labour of its accomplishment.

\section{List of Memoirs and Works.}

Ampère. Mémoire sur quelques propriétés nouvelles des axes permanens de rotation des corps, et des plans directeurs de ces axes. 4to. Paris, 1823.

Mémoire sur la Rotation. Mém. de l'Institut, t. v. 1834.

Mémoire sur les équations générales du mouvement. Liouv. t. I. pp. 211-228 (1836). (Written 1826.)

Anon. Note on the problem of falling bodies as affected by the earth's rotation. Camb. and Dubl. Math. Journ. t. III. pp. 206-208 (1848).

Remarks on the deviation of falling bodies to the east and south of the perpendicular, and corrections of a previously published paper on the same subject. Camb. and Dubl. Math. Journ. t. Iv. pp. 96-105 (1849).

Baehr. Notice sur le mouvement du pendule ayant égard à la rotation de la terre. 4to. Middelbourg, 1853.

Bertrand. Mémoire sur l'intégration des équations différentielles de la Mécanique. Liouv. t. xvII. pp. 393-436 (1852).

Note sur le Gyroscope de M. Foucault. Liouv. t. I. 2 sér. (1856), pp. 379-382.

Mémoire sur quelques-unes des formes les plus simples que puissent présenter les équations différentielles du mouvement d'un point matériel. Liouv. t. II. 2 sér. (1857), pp. 113-140.

Bessel. Analytische Auflösung der Keplerschen Aufgabe. Berl. Abh. 1816-17, pp. 49-55. (Read July 1818.)

- Ueber die Entwickelung der Functionen zweier Winkeln $u$ und $u^{\prime}$ in Reihen welche nach der Cosinussen und Sinussen der Vielfachen von $u$ und $u^{\prime}$ fortgehen. Berl. Abh. 1820-21, pp. 56-60. (Read June 1821.)

- Untersuchung des Theils der planetarischen Störungen welcher aus der Bewegung der Sonne entsteht. Berl. Abh. 1824, pp. 1-52.

Binet. Mémoire sur la théorie des axes conjugués et des momens d'inertie des corps. Journ. Polyt. t. Ix. (cah. 16), pp. 41-67 (1813). (Read May 1811.)

Note sur le mouvement du pendule simple en ayant égard à l'influence de la rotation diurne de la terre. Comptes Rendus, t. xxxir. (1851), pp. 157-160 and $197-205$. 
Bonnet. Note sur un théorème de Mécanique. Liouv. t. Ix. p. 113 (1844), and Note IV. of t. II. of the last edition of the Méc. Anal., pp. 329-331 (1855).

Booth. Theory of Elliptic Integrals. 8vo. Lond. $18 \check{1} 1$.

Bour. Mémoire sur le problème des trois corps. Journ. École Polyt. t. xxi. (cah. 36), pp. $35-58$ (1856).

Bravais. Mémoire sur l'influence qu'exerce la rotation de la terre sur le mouvement d'un pendule à oscillations coniques. Liouv. t. xIx. pp. 1-50 (1854).

- Note sur une formule de Lagrange relative au mouvement pendulaire. Note VII. of t. II. of the last edition of the Méc. Anal., pp. 352-355 (1855).

Briot. Thèse sur le mouvement d'un corps solide autour d'un point fixe. Liouv. t. vII. pp. $70-84$ (1842).

Cauchy. Sur les momens d'inertie. Exer. de Math. t. I. pp. 93-103 (1827).

- Résumé d'un mémoire sur la Mécanique Céleste et sur un nouveau calcul appelé des limites. (Read at Turin Oct. 1831.) Exer. d'Anal. \&c. t. II. pp. 41-109 (1841).

Cayley. On certain expansions in multiple sines and cosines. Camb. Math, Journ. t. III. pp. 162-167 (1842), [4].

- On the motion of rotation of a solid body. Camb. Math. Journ. t. IIr. pp. 224-232 (1842), [5].

- On certain results relating to quaternions. Phil. Mag. t. xxvi. (1845), p. 141, [20]. On the geometrical representation of the motion of a solid body. Camb. and Dubl. Math. Journ. t. I. pp. 164-167 (1846), [36].

. On the rotation of a solid body round a fixed point. Camb. and Dubl. Math. Journ. t. I. pp. 167-173 and 264-274 (1846), [37].

Note on a geometrical theorem in Prof. Thomson's memoir on the principal axes of a solid body. Camb. and Dubl. Math. Journ. t. I. pp. 207, 208 (1846), [38].

- On the application - of quaternions to the theory of Rotation. Phil. Mag. t. XXXIII. (1848), p. 196, [68].

Note on the motion of rotation of a solid of revolution. Camb. and Dubl. Math. Journ. t. IV. pp. 268-271 (1849), [78].

Sur les déterminants gauches. Crelle, t. xxxviıI. (1849), pp. 93-96, [69].

Note on the theory of Elliptic Motion. Phil. Mag. t. XI. (1856), pp. 425-428, [199].

A demonstration of Sir W. R. Hamilton's theorem of the Isochronism of the Circular Hodograph. Phil. Mag. t. XIII. (1857), p. 427, [209].

- Report on the recent progress of Theoretical Dynamics. Rep. Brit. Assoc. for 1857, pp. 1-42, [195].

C. IV. 
Cayley. On Lagrange's solution of the problem of two fixed Centres. Quart. Math. Journ. t. II. pp. $76-82$ (1858), [182].

. Note on the expansion of the true anomaly. Quart. Math. Journ. t. II. pp. 229-232 (1858), [191].

Tables in the theory of Elliptic Motion. Mem. R. Astr. Soc. t. xxIx. (1860), pp. $191-306,[216]$.

A Memoir on the problem of the rotation of a solid body. Mem. R. Astr. Soc. t. XxIx. (1860), pp. 307-342, [217].

. On Lambert's theorem for Elliptic Motion. Monthly Not. R. Astr. Soc. t. xxIr. pp. 238-242, (1861), [222].

- Note on a theorem of Jacobi's in relation to the problem of three bodies. Monthly Not. R. Astr. Soc. t. XI. pp. 76-79 (1861), [220].

Chasles. Note sur les propriétés générales du système de deux corps semblables entr'eux et placés d'une manière quelconque dans l'espace, et sur le déplacement fini ou infiniment petit d'un corps solide libre. (Read Feb. 1831.) Bull. Univ. des Sciences (Férussac), t. xIv. pp. 321-326.

. Théorèmes généraux sur les systèmes de forces et leurs moments. Liouv. t. xII. pp. 213-224 (1847).

Clairaut. Théorie de la Lune déduite du seul principe de l'attraction réciproquement proportionnelle aux carrés des distances. 4to. St Pét. 1752, and Paris, 1765.

Cohen. On the Differential Coefficients and Determinants of Lines, and their Application to Theoretical Mechanics. Phil. Trans. t. 152 (1862), pp. 469-510.

Cotes. Harmonia mensurarum sive analysis et synthesis per rationum et angulorum mensuras promotæ; accedunt alia opuscula mathêmatica. 4to. Camb. 1722.

Cournot. Mémoire sur le mouvement d'un corps rigide soutenu par un plan fixe. Crelle, t. v. pp. 133-162 and 223-249 (1830); Suite, t. viII. pp. 1-12 (1832).

Creedy. General and practical solution of Kepler's Problem. Quart. Math. Journ. t. I. pp. $259-271$ (1855).

D’Alembert. Traité de Dynamique. Paris, 1743.

- Recherches sur la précession des équinoxes et sur la nutation de l'axe de la terre. Mém. de Berl. (1749).

Desboves. Thèse sur le mouvement d'un point matériel attiré en raison inverse du. carré des distances vers deux centres fixes. Liouv. t. xIII. pp. 369-396 (1848).

Donkin. On an application of the calculus of operations to the transformation of trigonometric series. Quart. Math. Journ. t. II. pp. 1-15 (1858).

. On a class of Differential Equations, including those which occur in Dynamical Problems. Part I. Phil. Trans. t. cxliv. (1854), pp. 71-113; Part II. t. cxuv. (1855), pp. 299-358. 
Droop. On the Isochronism of the Circular Hodograph. Quart. Math. Journ. t. I. (1856), pp. $374-378$.

Dumas. Ueber die Bewegung des Raumpendels mit Rucksicht auf die Rotation der Erde. Crelle, t. L. pp. 52-78 and 126-185 (1855).

Durège. Theorie der elliptischen Functionen. 8vo. Leipzig, 1861. (§ xx. reproduces some results on the spherical pendulum obtained in an unpublished memoir of 1849.)

Euler. Determinatio Orbitæ Cometæ anni 1742. Misc. Berl. t. vIr. (1743), p. 1.

Theoria motuum planetarum et cometarum. 4to. Berl. 1744.

. De motu corporis ad duo virium centra attracti. Nov. Comm. Petrop. t. x. for 1764, pub: 1766 , pp. 207-242.

Problème: un corps étant attiré en raison réciproque carrée des distances vers deux points fixes donnés, trouver les cas où la courbe décrite par ce corps sera algébrique. Mém. de Berl. for 1760, pub. 1767, pp. 228-249.

. De motu corporis ad duo centra virium fixa attracti. Nov. Comm. Petrop. t. xI. for 1765 , pub. 1767 , pp. $152-184$.

- Considerationes de motu corporum cœlestium. Nov. Comm. Petrop. t. x. for 1764, pub. 1766 , pp. 544-558.

De motu rectilineo trium corporum se mutuo attrahentium. Nov. Comm. Petrop. t. XI. for 1765 , pub. 1767 , pp. 144-151.

De motu trium corporum se mutuo attrahentium super eâdem lineâ rectâ. Nov. Acta Petrop. t. III. (1776), pp. 126-141.

Problema algebraicum ob affectiones prorsus singulares memorabile. Nov. Comm. Petrop. t. xv. (1770), p. 75 ; Comm. Arith. Coll. t. I. pp. 427-443.

Formulæ generales pro translatione quâcunque corporum rigidorum. Nov. Comm. Petrop. t. xx. 1775 , pp. $189-207$.

. Nova methodus motum corporum rigidorum determinandi. Nov. Comm. Petrop. t. xx. (1775), p. 208.

Recherches sur la précession des équinoxes et sur la nutation de l'axe de la terre. Mém. de Berl. t. v. for 1749, pub. 1751, pp. 326-338. (Euler meńtions, t. vI., that this was written after he had seen D'Alembert's memoir.)

Découverte d'an nouveau principe de Mécanique. Mém. de Berl. t. vi. for 1750 , pub. 1752 , pp. $185-217$.

- Recherches sur la connaissance mécanique des corps. Mém. de Berl. for 1758 , pub. 1767 , pp. $132-153$.

. Du mouvement de rotation des corps solides autour d'une axe variable. Mém. de Berl. for 1758 , pub. 1765 , pp. 154-193. 
Euler. Du mouvement d'un corps solide lorsqu'il tourne autour d'une axe mobile. Mém. de Berl. for 1760, pub. 1767, pp. 176-227.

Theoria motus corporum solidorum. 4to. Rostock, 1765.

Foucault. Démonstration physique du mouvement de rotation de la terre au moyen du pendule. Comptes Rendus, t. xxxII. (1851), pp. 135-138.

Gauss. Fundamental-Gleichungen für die Bewegung schwerer Körper auf der rotirenden Erde, 1804.

- Theoria motus corporum cœlestium. 4to. Hamb. 1809. [Werke, t. vII. Gotha, 1871.]

Greatheed. Investigation of the general term of the expansion of the true anomaly in terms of the mean. Camb. Math. Journ. t. I. pp. 228-232 (1838).

Gudermann. De pendulis sphæricis et de curvis quæ ab ipsis describuntur sphæricis. Crelle, t. xxxviII. pp. 185-215 (1849).

Hamilton, Sir w. R. A theorem of anthodographic isochronism. Proc. R. Irish Acad. 1847, t. III. pp. $465-466$.

- Lectures on Quaternions, 8vo. Dublin, \&c. (1853).

Hansen. Fundamenta Nova investigationis orbitæ veræ quam Luna perlustrat. 4to. Gothæ, 1838.

- Ermittelung der absoluten Störungen in Ellipsen von beliebigen Excentricität und Neigung. Gotha, 1843, pp. 1-167.

Entwickelung des Products einer Potenz des Radius-Vectors mit dem Sinus oder Cosinus eines Vielfachen der wahren Anomalie in Reihen die nach den Sinussen oder Cosinussen der Vielfachen der wahren excentrischen oder mittleren Anomalie fortschreiten. Abh. d. K. Sächs. Ges. zu Leipzig, t. II. pp. 183-281 (1853).

- Entwickelung der negativen und ungeraden Potenzen der Quadratwurzel der Function $r^{2}+r^{\prime 2}-2 r r^{\prime}\left(\cos U \cos U^{\prime}+\sin U \sin U^{\prime} \cos J\right)$. Abh. d. K. Sächs. Ges. zu Leipzig, pp. 286-376 (1854).

Theorie der Pendelbewegung. 4to. Dantzig, 1856.

Haton de la Goupillière. Mémoire sur une théorie nouvelle de la géométrie des masses. Journ. École Polyt. t. Xxi. (cah. 37), 1858, $1^{\mathrm{r}}$ Mémoire, pp. 35-72; $2^{\mathrm{d}}$ Mémoire, pp. 73-96.

Haughton. On the rotation of a solid body round a fixed point, being an account of the late Professor Maccullagh's lectures on that subject, Hilary Term, 1844, in Trinity College, Dublin; compiled by the Rev. S. Haughton. Trans. R. Irish Acad. t. xxII. (1849), pp. 1-18.

Jacobi. Euleri formulæ de transformatione coordinatarum. Crelle, t. II. pp. 188, 189 (1827). 
Jacobi. Zur Theorie der Variations-Rechnung und der Differential-Gleichungen. Crelle, t. XViI. (1837), pp. 68-82. (1836).

De motu puncti singularis. Crelle, t. xxIv. pp. 5-27 (1842).

Elimination des nœuds dans le problème des trois corps. Crelle, t. xxvI. (1843), pp. 115-131.

- Theoria novi multiplicatoris systemati æquationum differentialium vulgarium applicandi ( $\$ 26$, two centres). Crelle, t. xxIx. pp. 333-337 (1845).

Sur la rotation d'un corps. Extrait d'une lettre adressée à l'Académie des Sciences. Comptes Rendus, t. xxIx. p. 97 ; and Liouv. t. xIv. pp. 337-344 (1849).

t. Xxхıx. pp. 293-350 (1850).

Nova methodus æquationes differentiales partiales primi ordinis inter numerum variabilium quemcunque propositas integrandi (posthumous, edited by $\mathbf{A}$. Clebsch). Crelle, t. Lx. pp. 1-181 (1862).

Lagrange.- Mécanique Analytique. 1st ed. 1788; 2nd ed. t. I. 1811; t. II. 1815; 3rd ed. 1855.

Sur une manière particulière d'exprimer le temps dans les sections coniques décrites par des forces tendantes au foyer et réciproquement proportionnelles aux carrés des distances. Mém. de Berlin for 1778; and Note V. of t. II. of the 3rd edition of the Méc. Anal. pp. 332-349.

Recherches sur le mouvement d'un corps qui est attiré vers deux centres fixes. Premier Mémoire, où l'on suppose que l'attraction est en raison inverse des carrés des distances. Anc. Mém. de Turin, t. Iv. (1766-1769), pp. 118-215.

hypothèses d'attraction. Anc. Mém. de Turin, t. IV. (1766-1769), pp. 215-243.

Nouvelle solution du problème du mouvement de rotation d'un corps. Mém. de Berl. for 1773 .

Lambert. Insigniores Orbitæ Cometarum Proprietates. 8vo. Aug. 1765.

Laplace. Mécanique Céleste, t. I. 1799 ; t. II. 1799 ; t. III. 1802 ; t. IV. 1805 ; t. v. 1823.

Mémoire sur le développement de l'anomalie vraie et du rayon vecteur elliptique en séries ordonnées suivant les puissances de l'excentricité. Mém. de l'Inst. t. vi. (1823), pp. $63-80$.

Lefort. Expressions numériques des intégrales définies qui se présentent quand on cherche les termes généraux des développements des coordonnés d'un planète dans son mouvement elliptique. Liouv. t. XI. pp. 142-152 (1846). 
Legendre. Exercices de Calcul Intégrale, t. II. (containing the dynamical applications) 1817.

Traité des Fonctions Elliptiques, t. I. (1825) (but the dynamical applications are for the most part reproduced from the Exercices).

Leverrier. Annales de l'Observatoire de Paris, t. I. (1855).

Lexell. Theoremata nonnulla generalia de translatione corporum rigidorum. Nov. Comm. Petrop. t. xx. (1775), pp. 239-270.

Liouville. Sur l'intégrale $\int_{0}^{\pi} \cos i(u-x \sin u) d u$. Liouv. t. vi. pp. 36,37 (1841).

—. Extrait d'un mémoire sur un cas particulier du problème de trois corps. Liouv. t. viI. pp. 110-113 (1842).

Sur quelques cas particuliers où les équations du mouvement d'un point matériel peuvent s'intégrer. 3 Memoirs. Liouv. t. XI. pp. 345-379 (1846), t. XII. pp. 410-44 (1847), and t. xIv. pp. 257-300 (1849).

—. Mémoire sur un cas particulier du problème de trois corps. Conn. des Temps for 1845, and Liouv. t. I. (2 sér.), pp. 248-256 (1842).

- Note ajoutée au mémoire de M. Serret (two revolving centres). Liouv. t. XIII. pp. 34-37 (1848).

Lottner. Reduction der Bewegung eines schweren um einen festen Punct rotirenden Revolutions-Körpers auf die elliptischen Transcendenten. Crelle, t. L. pp. 111-125 (1855).

—. Zur Theorie des Foucaultschen Pendelversuchs. Crelle, t. LII. pp. 52-58 (1856). Maccullagh, see Haughton.

Möbius. Ueber die Zusammensetzung unendlich kleiner Drehungen. Crelle, t. xvinI. pp. 189-212 (1838).

- Der barycentrische Calcul. 8vo. Leipzig, 1827.

Lehrbuch der Statik. 8vo. Leipzig, 1837.

Die Elemente der Mechanik des Himmels. 8vo. Leipzig, 1843.

Newton (Sir Isaac). Philosophiæ Naturalis Principia Mathematica. 4to. London, 1687.

Pagani. Démonstration d'un théorème de Lambert. Crelle, t. xv. pp. 350—352 (1834).

- Mémoire sur l'équilibre d'un corps solide suspendu à un cordon flexible. Crelle, t. xxix. pp. 185-204 (1839).

Painvin. Recherche du dernier multiplicateur pour deux formes spéciales et remarquables des équations différentielles du problème de trois corps. Liouv. t. xIx. pp. 88-111 (1854).

Poinsot. Mémoire sur la rotation. Extrait, 8vo. Paris, 1834. 
Poinsot. Théorie nouvelle de la rotation des corps. Liouv. t. xvi. pp. 9-130 and 289336 (1851).

. Théorie des cônes circulaires roulants. Liouv. t. xvIII. pp. 41-70 (1853).

- Questions dynamiques sur la percussion des corps. Liouv. t. II. (2 sér.), pp. $281-329$ (1859).

Poisson. Traité de Mécanique. 1st ed. Paris, 1811; 2nd ed. Paris, 1833.

. Mémoire sur un cas particulier du mouvement de rotation des corps graves. Journ. École Polyt. t. Ix. (cah. 16), pp. 247-262 (1813).

- Sur une nouvelle manière d'exprimer les coordonnés des planètes dans le mouvement elliptique. Conn. des Temps for 1825, pp. 379-386.

Sur la développement des coordonnés d'une planète dans son mouvement elliptique et de la fonction perturbative de ce mouvement. Conn. des Temps for 1836, pp. 3-31.

. Mémoire sur le mouvement d'un corps solide (read 1834). Mém. de l'Inst. t. xIv. pp. $275-432$ (1838).

Sur le mouvement des projectiles en ayant égard à la rotation de la terre. Journ. École Polyt. t. xvi. pp. 1-68 (1838).

- Mémoire sur le mouvement des projectiles dans l'air, en ayant égard à leur rotation. Journ. École Polyt. pp. 69-176 (1838).

Prony. Analyse détaillée des différentes questions qui se rapportent au mouvement d'un corps sollicité par des puissances quelconques. Journ. École Polyt. t. Iv. (cah. 11), pp. 87-143 (1802).

Puiseux. Note sur le mouvement d'un point matériel pesant sur une sphère. Liouv. t. vII. pp. $517-520$ (1842).

$\mathrm{Du}$ mouvement d'un solide de révolution posé sur un plan horizontal. Liouv. t. XIII. pp. 249-256 (1848).

- Sur la convergence des séries qui se présentent dans la théorie du mouvement elliptique des planètes. Liouv. t. xIv. pp. 33-39 (1849).

Seconde Note sur la convergence des séries du mouvement elliptique. Liouv. t. xIv. pp. 247,248 (1849).

. Solution de quelques questions relatives au mouvement d'un corps solide pesant posé sur un plan horizontal. Liouv. t. xviI. pp. 1-30 (1852). qui se présentent dans la théorie du mouvement elliptique. Méc. Anal. 3rd ed t. II. (Note I.), pp. 313-317 (1855). 
Quet. Des mouvements relatifs en général, et spécialement des mouvements relatifs sur la terre. Liouv. t. xvIII. pp. 213-298 (1853).

Richelot. Bemerkung über einen Fall der Bewegung eines systems von materiellen Puncten. Crelle, t. XL. pp. 178-182 (1850).

- Auszug eines Schreibens am Herrn Prof. Jacobi. Crelle, t. xLII. pp. 32-34 (1851).

- Bemerkungen zur Theorie des Raumpendels. Crelle, t. xLII. pp. 233-238 (1853).

- Eine neue Lösung des Problems der Rotation eines festen Körpers um einen Punct. (Auszug einer zu Berlin, 1851, erschienenen Schrift.) Crelle, t. xuIv. pp. $60-65$.

Rodrigues. Des lois géométriques qui régissent les déplacements d'un système solide dans l'espace, et de la variation des coordonnés provenants de ces déplacements considérés indépendamment des causes qui peuvent les produire. Liouv. t. III. pp. $380-440$ (1840).

Rueb. Specimen inaugurale de motu gyratorio corporis rigidi nullâ vi acceleratrice sollicitati. Utrecht, 1834.

Schellbach. Mathematische Miszellen. No. I.-IV. Ueber die Bewegung eines Puncts der von einem festen Puncte angezogen wird. Crelle, t. xLV. pp. 255-266 (1853).

Schubert. Astronomie Théorique. 4to. St Pét. (1822).

Segner. Specimen theoriæ turbinum. 4to. Halæ, 1755.

Serret. Thèse sur le mouvement d'un point attiré par deux centres fixes en raison inverse du carré des distances. Liouv. t. xıII. pp. 17-33 (1848).

- Sur la solution particulière que peut admettre le problème du mouvement d'un corps attiré vers deux centres fixes par des forces réciproquement proportionnelles aux carrés des distances. Note III. to t. II. of the 3rd ed. of the Méc. Anal. pp. $327-331$ (1855).

Sohncke. Motus corporum cœlestium in medio resistente. Crelle, t. x. pp. 23-40 (1833).

Somoff. Démonstration des formules de M. Jacobi relatives à la théorie de la rotation d'un corps solide. Crelle, t. XLII. pp. 95-116 (1851).

Stader. De orbitis et motibus puncti cujusdam corporei circa centrum attractivum aliis quam Neutonianâ attractionis legibus sollicitati. Crelle, t. xLvi. pp. 262--327 (1852).

Steichen. De la propriété fondamentale du mouvement cycloidal, et de sa liaison avec le principe de la composition des mouvements de rotation autour des axes parallèles et des axes qui se coupent. Crelle, t. xlvi. pp. 24-42 (1853).

- Mémoire sur la question réciproque du centre de percussion. Crelle, t. xlviII. pp. $1-68$ (1854). 
Steichen. Mémoire de Mécanique relatif au mouvement de rotation et au mouvement naissant des corps solides. Crelle, t. xLIII. pp. 161-244 (1852), and Addition t. XLIV. pp. $43-46$ (1853).

Sylvester. Sur l'involution des lignes droites dans l'espace considérées comme des axes de rotation. Comptes Rendus, t. LII. (1861), p. 741.

. Note sur l'involution de six lignes dans l'espace. Comptes Rendus, t. LII. (1861), p. 815 .

Thomson. On the principal axes of a solid body. Camb. and Dubl. Math. Journ. t. I. pp. 127-133 and 195-206 (1846).

Tissot. Thèse de Mécanique. Liouv. t. XVII. pp. 88-116 (1852).

Townsend. On the principal axes of a solid body, their moments of inertia and distribution in space. Camb. and Dubl. Math. Journ. t. I. pp. 209-227, and t. II. pp. $19-42,140-171$ and $241-251$ (1846-47).

walton. Instantaneous lines and planes in a body revolving round a fixed point. Camb. and Dubl. Math. Journ. t. vII. pp. 111-113 (1852).

Whewell. A Treatise on Dynamics. 1st ed. Cambridge, 1823.

worms. The Earth and its Mechanism-being an account of the various proofs of the rotation of the Earth, with a description of the instruments used in the experimental demonstrations: to which is added the theory of Foucault's Pendulum and Gyroscope. 8vo. London, 1862. 